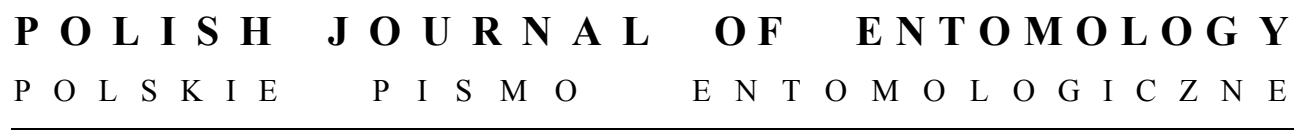

VOL. 81: 211-304

Gdańsk

30 September 2012

DOI: $10.2478 / \mathrm{v} 10200-012-0009-8$

\title{
Biting midges of the genus Dasyhelea KIEFFER (Diptera: Ceratopogonidae) in Poland
}

\author{
PATRYCJA DOMINIAK
}

Department of Invertebrate Zoology and Parasitology, University of Gdańsk, Wita Stwosza 59, 80-308 Gdańsk, Poland, e-mail: heliocopris@gmail.com

\begin{abstract}
This article reviews 30 species of biting midges of the genus Dasyhelea that are known to occur in Poland. Diagnoses and detailed descriptions of adults are provided for all Polish species, as well as keys (based mainly on males) for the identification of subgenera and their included species. Dasyhelea excellentis BORKENT, 1997 is recognized as a new junior synonym of Dasyhelea bifida ZILAHI-SEBESS, 1936 (syn. n.) and Dasyhelea lugensis BRODSKAYA, 1995 as a new junior synonym of Dasyhelea caesia REMM, 1993 (syn. n.).

Among the Polish biting midge fauna, most species of the genus Dasyhelea are primarily arboreal, widely distributed Palaearctic species. The most species-rich samples were collected in the following regions: Eastern Baltic Lakelands (19 spp.), Southern Baltic Lakelands (16 spp.), Southern Baltic Coastlands (14 spp.) and Central Western Carpathians (12 spp.). The larvae were found in sap and tree holes, water accumulated in gutters and sinks, moist soil near fresh- and saltwater bodies, and peat bogs. Adults are known to visit flowers for nectar, and 16 Polish species were found on umbelliferous flowers.
\end{abstract}

KEY WORDS: Diptera, Ceratopogonidae, Dasyhelea, biting midges, distribution, key, new synonyms, Poland.

\section{INTRODUCTION}

The biting midges (Diptera: Ceratopogonidae) are a family of small nematocerous flies that are morphologically very similar to non-biting midges (Chironomidae). There are nearly 6100 known valid extant and fossil species, belonging to 134 genera and 5 subfamilies: Lebanoculicoidinae, Leptoconopinae, Forcipomyiinae, Dasyheleinae and Ceratopogoninae (BORKENT 2012). In Poland this family is represented by 218 species and 21 genera (SZADZIEWSKI 2007, SZADZIEWSKI et al. 2007a,b, DominiAK \& SZADZIEWSKI 2008, DOMINIAK \& MichaLCZUK 2009, DOMINIAK \& SZADZIEWSKI 2010). 
The genus Dasyhelea includes biting midges of diverse morphology and biology (WAUGH \& WIRTH 1976) and has been recorded from all regions of the World except Antarctica (GROGAN \& WIENERS 2006). Currently, 612 valid species of Dasyhelea have been described, including 14 fossil species (STROBL 1910, BORKENT 2012). In Europe, Dasyhelea is represented by 63 species, with 30 known to occur in Poland (DOMINIAK \& SZADZIEWSKI 2010). Unlike most other ceratopogonids, there are no predaceous or ectoparasitic species in Dasyhelea as females have reduced, vestigial mandibles and maxillae that lack teeth. However, like other ceratopogonids, adult males and females feed on honeydew and nectar as sources of energy. Some tropical species are pollinators of plants, e.g. cocoa trees, Theobroma cacao L. and rubber trees, Hevea brasiliensis (WILLD. ex Adr. Juss.) Muell. Arg (LeE et al. 1989). Our knowledge of the biology and phylogenetics of Dasyhelea midges is relatively poor. The preimaginal stages of most species are unknown (BRODSKAYA 1995) and the relationships between species, species groups and subgenera still remain unclear (SZADZIEWSKI 1985a).

\section{Biology}

The immature stages of many species of Dasyhelea live in algae, mosses or liverworts and among plants on water margins. They inhabit puddles, springs, swamps, peat-bogs and moist soil. Midges of this genus are quite common in phytotelmata, for example in tree hollows, damaged tree trunks and stems of bamboo, fruit shells and leaf axils (JOHANNSEN 1931, MAYer 1934a,c, Zilahi-Sebess 1936b, PrZhiboro 2005, DominiaK \& SZADZIEWSKI 2006), e.g. common teasel Dipsacus sylvestris HUDS. (GOETGHEBUER 1925, DISNEY \& WIRTH 1982). In terrestrial habitats Dasyhelea larvae can live in sap oozing from trees, rotting plants or mushrooms (WAUGH \& WIRTH 1976, GRAVES \& GRAVES 1985) and in faeces (VATTIER 1964). Some species are reported from hot springs with a high mineral content or low $\mathrm{pH}$, and pitchers of carnivorous plants of the genus Nepenthes LiNNAEUS (JohANNSEN 1931, MAYer 1934c, ShePpe 1973, WirTh \& BEAVER 1979, LeE \& ChAN 1985). Several species inhabit ephemeral rock pools or their artificial equivalents, rain gutters, float systems, open drainage channels, stoups, etc. (KIEFFER 1925c, JOHANNSEN 1931, Zilahi-Sebess 1931, REMMERT 1953, WirTh 1987, LeE et al. 1989, Ashe et al. 2007). Some Dasyhelea larvae live in saline soil or water of inland saline habitats and intertidal zone of seas (THIENEMANN 1925, MAYER 1934a, WIRTH 1978). There are several halobiontic, halophilic and haloxenic species of Dasyhelea (SZADZIEWSKI 1983).

The eggs of Dasyhelea are unique in being horseshoe-shaped (Fig. 29) and are laid in loose masses enclosed in a gelatinous coating (MUNSTREHJELM 1920, KEILIN 1921, WAUGH \& WIRTH 1976, RondEROS et al. 2006, MARTínEZ et al. 2010).

All larvae of Ceratopogonidae, including Dasyhelea, are apneustic in all four instars (SzADZIEWSKI et al. 1997). Larvae of some Dasyhelea species are well adapted to survive 
long periods of desiccation, temperature fluctuations, low oxygen levels, digestive enzymes of insectivorous plants and low pH (ZiLAhI-Sebess 1931, Disney 1975, LeE \& CHAN 1985). They cannot swim, but move slowly by using their mandibles to pull and posterior hooks to push themselves forward (WIRTH 1978, FÜRST VON LIEVEN 1998) (Fig. 125). Most North American species spin tubular cases in the last instar (WAUGH \& WIRTH 1976). Among European Dasyhelea, this behaviour has been observed in the larvae of D. modesta (WINNERTZ) (THIENEMANN 1915).

The morphology of the larval head capsule as well as field and laboratory observations indicate that most Dasyhelea larvae are primarily herbivorous (MULLEN \& HRIBAR 1988, FÜRST VON LIEVEN 1998, RONDEROS et al. 2003). However, it is more likely that Dasyhelea larvae exploit a wide variety of food items. They feed on green algae, diatoms, fungi and detritus (ZILAHI-Sebess 1931, MulLen \& Hribar 1988). Dasyhelea larvae can be carnivorous or scavengers, as confirmed by the presence of oligochaete setae in their alimentary tracts (MULLEN \& HRIBAR 1988). Research by ZILAHI-SEBESS (1931), DODSON (1987), LeE \& ChAN (1985) and Hribar \& Denson (2008) suggests that larvae prey on protozoans, rotifers, chironomid larvae and injured or dead mosquito larvae.

Adults typically live only a few days (ZILAHI-SEBESS 1931) and are usually found in or near breeding sites, among shrubs or herbs and on flowers (WAUGH \& WIRTH 1976). They are especially common on umbelliferous plants (SZADZIEWSKI et al. 1997), but have also been recorded on birthwort, Aristolochia clematitis L. (HAVELKA 1978), and the flowers of cocoa and rubber trees (LeE \& ChAN 1985). Adult male Dasyhelea fly in swarms (BORKENT \& SPINELLI 2007), but such behaviour has not been observed in many species (LEE \& CHAN 1985). The structure of the mouthparts is the same in both sexes (CARTER et al. 1921, Glukhova 1981) - males and females of Dasyhelea feed on nectar and honeydew, or do not feed at all (ZILAHI-SeBess 1931, LeE \& CHAN 1985).

\section{Classification}

In his classic study on the Ceratopogonidae of California, USA, WIRTH (1952) divided Dasyhelea into four species groups - cincta, grisea, mutabilis and traverae. He chose to avoid subgenera for North American species because of the poor understanding of both faunistics and phylogenetic relationships among the species. Subsequently, several new species groups were proposed: leptobranchia, which replaced the traverae group (WAUGH \& WIRTH 1976), borgmeieri (WIRTH \& WAUGH 1976) and brevicornis (GROGAN \& WIENERS 2006). Recently, DíAZ et al. (2010) proposed the patagonica group for a few southern Neotropical species of Dasyhelea. Most European workers on Dasyhelea have adopted the five subgenera arrangement by REMM $(1962,1979)$. In addition to Dasyhelea s. str. and Prokempia KIEFFER, Remm also regarded Pseudoculicoides Malloch, Dicryptoscena ENDERLEIN and Sebessia REMM as subgenera. According to REMM's (1979) 
system, the cincta group belongs to the subgenus Sebessia together with the holosericea group (proposed by REMM, ibid.), the grisea group correlates well with Dasyhelea s. str., while the mutabilis group along with the borgmeieri group correlate well with Pseudoculicoides (REMm 1962, WAUGH \& WIRTH 1976).

\section{Studies of Polish Dasyhelea midges}

The first report on Dasyhelea in Poland was written by MAYER (1934b), in which D. holosericea (MeIGEN) and D. notata GoETGHebuer are mentioned from the southwestern region of the country. However, because both of these records were based on females, they are suspect and should be ignored. KARL (1940) reported $D$. holosericea and D. modesta (WINNERTZ) (as D. tarsalis KIEFFER) from the environs of Słupsk (voucher male specimens in MZPW). SZADZIEWSKI (1983) mentioned seven species of Dasyhelea from a variety of saline habitats, including six species new to the Polish fauna. In his revision of the Palaearctic species of the johannseni group, SZADZIEWSKI (1985a) provided new locality records for $D$. turficola KIEFFER, and, one year later, additional records of D. modesta (SZADZIEWSKI 1986). Moreover, in his 1986 article, SZADZIEWSKI reported the first Polish records of $D$. notata, D. thienemanni Spătaru \& DAmian-GeORgescu, D. bilobata KiefFer (as D. luteiventris Goetghebuer) and $D$. flavoscutellata (ZeTtERSTEDT). As many as fifteen Dasyhelea species were listed in the Checklist of Animals of Poland, among them three species new to the Polish fauna (SZADZIEwSKI 1991). A few years later, a species associated with tree sap, D. flavifrons (GUÉRIN) (as D. versicolor (WINNERTZ)), was reported from Poland for the first time (KACZOROWSKA 1996). In 2001, a new locality was reported for D. notata in the Białowieża Forest (SZADZIEWSKI 2001), and shortly thereafter, the first Polish records were published of D. bicrenata KIEFFER (SZADZIEWSKI \& BORKENT 2003), D. malleola REMM (SZADZIEWSKI et al. 2004) and D. bilineata GoetgheBUer (as D. saxicola (EDWARDS)) (SZADZIEWSKI \& DOMINIAK 2006). Some information on Dasyhelea midges occurring in peat bogs in northern and southern Poland was given in articles by DOMINIAK and co-workers (DOMINIAK \& SZADZIEWSKi 2006, DOMINIAK et al. 2007b, DominiaK 2009). Finally, a few species of Dasyhelea were reported for the first time from this country in the second volume of the Fauna of Poland (SZADZIEWSKI 2007) and in three articles by DOMINIAK \& SZADZIEWSKI (2006, 2008, 2010). Recently, GwIZDALSKA-KENTZER (2011) supplied new locality records for five species $-D$. bilineata, $D$. flavifrons, $D$. flavoscutellata, D. modesta and D. pallidiventris (GOETGHEBUER) - found during an investigation of the biting midges of the Łęg nad Swelinią Reserve in northern Poland.

In comparison to other European countries, biting midges of the genus Dasyhelea are relatively well known in Poland, with 30 species known from this country. The same number of Dasyhelea species is also known from Estonia, where the Ceratopogonidae 
fauna was intensively studied by KIEFFER and soon after by REMM (DOMINIAK \& SZADZIEWSKI 2010).

\section{Acknowledgements}

I am obliged to all who helped me with arranging loans of the materials, and sent me information on type specimens as well as some missing publications. Many thanks are due to Art Borkent (Salmon Arm, Canada), Peter J. Chandler (Melksham, Great Britain), JeanClaude Delécolle (University of Strasbourg, France), Florentina Díaz (La Plata Museum, Argentina), William L. Grogan (Florida State Collection of Arthropods, USA), Lawrence J. Hribar (Florida Keys Mosquito Control District, USA), Pol Limbourg (Royal Belgian Institute of Natural Sciences, Belgium), Jaan Luig (University of Tartu Natural History Museum, Estonia), Bruno Mathieu (University of Strasbourg, France), Athol McLachlan (Newcastle University, Great Britain), Walter Pfliegler (Hungary), Andrey A. Przhiboro (Zoological Institute of the Russian Academy of Sciences, Russia), Gustavo R. Spinelli (Museo de La Plata, Argentina) and Andrea Tóthová (Masaryk University, Czech Republic). I am very grateful to the reviewers, William L. Grogan and Ryszard Szadziewski (University of Gdańsk, Poland), for their valuable comments, and to Elżbieta Sontag (University of Gdańsk, Poland), for her help in preparing the manuscript.

Studies on Dasyhelea were supported by the Polish Ministry of Science and Higher Education (project No. N303 054 31/1710) and the University of Gdańsk (BW projects Nos. 1130-5-0223-4, 1430-5-0253-5 and 1430-5-0024-6).

\section{MATERIALS AND METHODS}

A total of 4256 specimens of Dasyhelea, including 2318 adult males and 1657 females, 258 pupae or pupal exuviae and 23 larvae were examined for this study. Species identification was based mainly on adult males.

Nearly all the Dasyhelea midges used in this study were collected in Poland between 1912 and 2011 and are currently deposited in the Department of Invertebrate Zoology and Parasitology, University of Gdańsk. The abbreviations of the collectors' names are as follows: (K.B.) Katarzyna Bendarowicz, (Z.B.) Zbigniew Biliński, (M.C.) Mateusz Ciechanowski, (P.D.) Patrycja Dominiak, (K.G.) Katarzyna Grędzińska, (M.G.K.) Marta Gwizdalska-Kentzer, (E.K.) Elżbieta Kaczorowska, (W.K.) Wiesław Krzemiński, (J.K.) Jarosław Krzywiński, (R.L.) Renata Ludwińska, (Cz.M.) Czesława Majkowska, (G.O.) Gabriela Okrój, (K.P.) Katarzyna Podbielska, (B.S.) Barbara Skierska, (R.Sz.) Ryszard Szadziewski, (A.W.) Alina Warzocha. A few specimens were collected abroad or borrowed from the following collections: Museum and Institute of Zoology, Polish Academy of 
Sciences (MZPW), Royal Belgian Institute of Natural Sciences (IRSNB), University of Strasbourg, Zoological Museum (MZSF), University of Tartu, Natural History Museum (TUZ) and the U.S. National Museum of Natural History (USNM). The abbreviations of the collections are those used by BORKENT \& WIRTH (1997) and EVENHUIS (2012).

In some cases, the information from the original labels has been supplemented with data on sampling sites, dates, collectors, etc. (reported in square brackets).

Specimens were collected from 130 localities (Figs 1-2), situated in 95 quadrants of the UTM grid (reported in parentheses). The division into physiographic regions follows KONDRACKI (2000), with modifications suggested by SZADZIEWSKI (1985b).

\section{SOUTHERn BALTIC COASTLANDS (31 localities)}

Brzyno near Żarnowiec (CF07); Elblag (CF90); Gdańsk (CF43); Gdańsk Brzeźno (CF43); Gdańsk Jelitkowo (CF43); Gdańsk Oliwa (CF43); Gdańsk Politechnika (CF42); Gdańsk Stocznia (CF42); Gdańsk Wrzeszcz (CF42); Gdańsk, Sobieszewska Is., Górki Wschodnie (CF52); Gdańsk, Sobieszewska Is., Przegalina (CF62); Gdynia Orłowo (CF43); Gdynia Wzgórze Św. Maksymiliana (CF44); Kadyny near Elbląg (DF01); Karsibór on Uznam Is. (VV56); Kiezmark near Gdańsk (CF61); Krzewsk near Elblag (CE99); Lisewo near Tczew (CE59); Malbork (CE78); Mechelinki near Gdynia (CF35); Piaśnica near Wejherowo (CF16); Puck (CF36); Sopot (CF43); Sopot - Gdańsk Jelitkowo (CF43); Strzebielino Morskie near Wejherowo (CF05); Mieroszyno near Władysławowo (CF17); Wejherowo (CF25); Widzino near Słupsk (XA23); Wierzchucino near Żarnowiec (CF07); Władysławowo (CF37); Żarnowiec (CF17).

EASTERn BALTiC COASTLANDS (2 localities)

Barciany near Kętrzyn (EF20); Łęknica near Kętrzyn (EF31).

SOUTHERN BALTIC LAKELANDS (27 localities)

Aleksandrów Kujawski (CD46); Babi Dół near Kartuzy (CF22); Gdańsk, Dolina Radości (CF43); Gdynia Bernadowo (CF33); Gdynia Kacze Buki (CF33); Głowaczewo near Wałcz (XV00); Inowrocław Mątwy (CD15); Inowrocław Rąbin (CD15); Janikowo near Inowrocław (CD04); Lake Otalżyno near Kartuzy (CF13); Krzeszna near Kartuzy (CF01); Miastko near Bytów (XV28); Mirachowo near Kartuzy (CF03); Mrowiniec near Tuchola (XV85); Ocypel near Starogard Gdański (CE26); Okole near Starogard Gdański (CE38); Starogard Gdański (CE38); Głusk near Wałcz (WU68); Jaźwiny near Wałcz (WU69); Miechucino near Kartuzy (CF02); Sitnica near Wałcz (WU68); Załom near Wałcz, near Delta Płocicznej Reserve (WU68); Załom near Wałcz, near Lake Ostrowieckie (WU68); Szubin near Bydgoszcz (XU87); Szwecja near Wałcz (XV01); Toruń Barbarka (CD38); Żakowo near Sulęczyno (XA81).

\section{EASTERN BALTIC LAKELANDS (20 localities)}

Aleksiejówka near Sejny (FE59); Bachanowo near Suwałki (FF11); Ełk (EE86); Giżycko (EE58); Kętrzyn (EE29); Krzywe near Suwałki (FE39); Łopuchowo near Suwałki (FF21); Mazurki near Augustów (FE27); Michnowce near Sejny (FF40); Olsztyn (DE65); Orłowo near Olecko (EE79); 
Piecki near Mragowo (EE25); Płaska near Augustów (FE47); Poszeszupie near Suwałki (FF32); Rosochaty Róg near Suwałki (FE39); Silec near Kętrzyn (EF30); Solanka near Kętrzyn (EF30); Słupie near Suwałki (FE39); Wiatrołuża Pierwsza near Suwałki (FE39); Żytkiejmy near Gołdap (FF02).

Central Poland Lowlands (5 localities)

Chylice near Warszawa (EC07); Czeszów near Wrocław (XS59); Młochów near Warszawa (DC86); Pułtusk near Warszawa (ED14); Przasnysz near Warszawa (DD97).

PODLASIE (7 localities)

Białowieża (FD94); the hamlet of Maruszka on the Siemianówka Reservoir near Białystok (FD96); Kunicha near Augustów (FE35); Pogorzelce near Białowieża (FD84); Popiołówka near Białystok (FE41); Białowieża Forest (FD83); Sztabin near Augustów (FE35).

\section{SuDETY MTS. (6 localities)}

Karpacz (WS52); Pokrzywna near Głuchołazy (XR77); Sosnówka Dolna near Jelenia Góra (WS52); Pasterka near Kudowa Zdrój (WR99); Przełęcz Łącznik in the Izerskie Mts. (WS23); Radomierz near Jelenia Góra (WS63).

\section{SiLESIAN-KRAKÓw UPLAND (2 localities)}

Dolina Będkowska near Kraków (DA15); Ojców near Kraków (DA16).

CENTRAL MAŁOPOLSKa UPLAND (2 localities)

Święta Katarzyna near Kielce (DB94); Wola Szczygiełkowa near Kielce (DB93).

EASTERN MAŁOPOLSKA UPLAND (3 localities)

Zwierzyniec near Zamość (FB40); Brody Małe near Szczebrzeszyn (FB31); Kawęczyn near Szczebrzeszyn (FB31).

POLESIE (1 locality)

Libiszów near Włodawa (FC41).

OUter WeStern CARPATHIANS (2 localities)

Moszczenica near Żywiec (CA70); Zawoja Składy (CV99).

Central Western Carpathians (15 localities)

Czertezik in Pieniny Mts. (DV57); Dolina Małej Łąki in the Tatra Mts. (DV25); Dolina Strążyska in the Tatra Mts. (DV25); Krościenko on the River Dunajec (DV57); Przysłop Miętusi in the Tatra Mts. (DV15); Szafranówka in the Pieniny Mts. (DV67); Czarny Dunajec near Nowy Targ, Czarny Dunajec-Baligówka (DV18); Czarny Dunajec near Nowy Targ, Baligówka peat bog (DV18); Chyżne near Nowy Targ, Las Hamrzyska (DV07); Chyżne near Nowy Targ, Puścizna Jasiowska peat bog 
(DV07); Chyżne near Nowy Targ, Wyżni Młyn (DV07); Piekielnik near Nowy Targ (DV17); Sokolica in the Pieniny Mts. (DV57); Zakopane Huty (DV26); Zakopane Jaszczurówka (DV26).
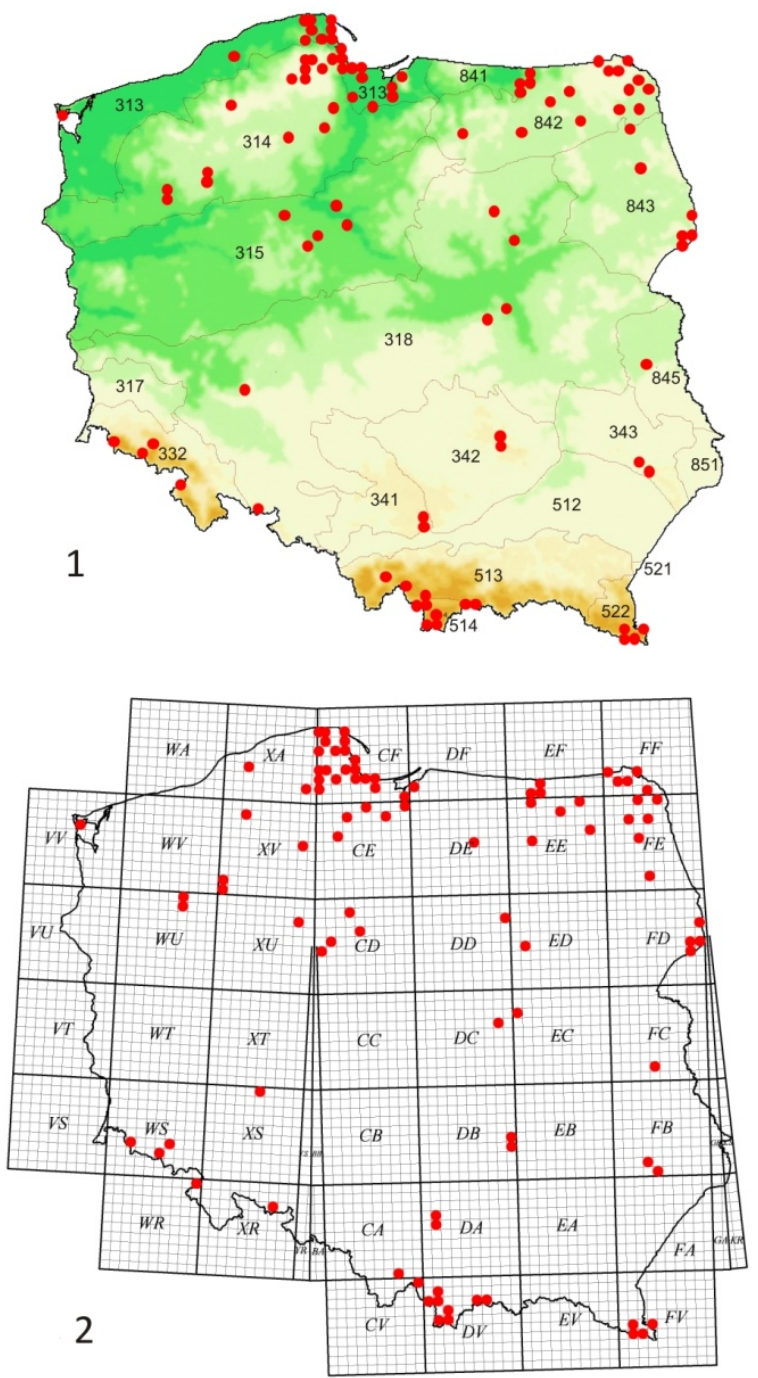

Figs 1-2. Sampling sites in Poland: 1 - physiographic regions; 2 - UTM grid map.

313 - Southern Baltic Coastlands, 314, 315 - Southern Baltic Lakelands, 317, 318 - Central Poland Lowlands, 332 - Sudety Mts., 341 - Silesian-Kraków Upland, 342 - Central Małopolska Upland, 343 - Eastern Małopolska Upland, 512 - Northern Podkarpacie, 513, 521 - Outer Western Carpathians, 514 - Central Western Carpathians, 522 - Eastern Beskid Mts., 841 - Eastern Baltic Coastlands, 842 - Eastern Baltic Lakelands, 843 - Podlasie, 845 - Polesie, 851 - Volhynian-Podolian Upland. 
EASTERN BESKID MTS. (7 localities)

Brzegi Górne near Ustrzyki Górne (FV14); Moczarne near Wetlina (FV14); Muczne near Ustrzyki Górne, near the Litmirz Reserve (FV34); Muczne near Ustrzyki Górne, Tarnawa Reserve (FV34); Wołosate near Ustrzyki Górne (FV23); Ustrzyki Górne (FV13); Ustrzyki Górne (FV23).

Most adult Dasyhelea were collected with an entomological net, only a few specimens were caught with light, Barber, Malaise or Moericke traps. Substrate samples were taken from various habitats and preimaginal stages were reared in the laboratory, adults being allowed to harden for 24 hours after emergence. All specimens were preserved in $75 \%$ ethanol, then subsequently cleared in phenol-ethanol and mounted on microscope slides in phenol-Canada balsam by the methods described in WIRTH \& MARSTON (1968). All descriptions, illustrations and measurements were based on slide-mounted specimens.

The Scanning Electron Microscope (SEM) images were produced at the Department of Plant Cytology and Embryology, University of Gdańsk (SPI-MODULE Sputter Coater, Philips XL 30 Scanning Electron Microscope) and at the Museum and Institute of Zoology, Polish Academy of Sciences (critical point dryer E3100 Jumbo, SC7640 Sputter Coater, HITACHI S-3400N Scanning Electron Microscope). Materials preserved in 75\% alcohol were dehydrated in ethanol series, dried in HMDS or critical-point dried and subsequently gold-coated.

Whenever possible, at least ten specimens were measured for each character $(\mathrm{n}=$ number of specimens examined). Sometimes, when there were few specimens from Poland, additional material collected in other European countries was also measured. The following special terms and their abbreviations are used in the descriptions:

- $\quad$ wing length - measured from the basal arculus to the wing tip [mm];

- CR (costal ratio) - length of the costal vein measured from the basal arculus to the tip of the second radial cell divided by the wing length;

- AR (antennal ratio) - male: combined length of distal four flagellomeres divided by combined length of proximal nine flagellomeres; female: combined length of distal five flagellomeres divided by combined length of proximal eight flagellomeres;

- $\quad \mathrm{PR}_{\text {(III) }}$ (palpal ratio) - length of palpal segment 3 divided by its greatest breadth;

- length of spermatheca - measurements included the neck [in $\mu \mathrm{m}]$;

- $\quad$ width of spermatheca - measured at its widest point [in $\mu \mathrm{m}]$.

\section{Data on geographical distribution}

Any literature reports that do not include detailed information on sampling sites, as well as doubtful records, are not included in the summaries of distribution data. 


\section{RESULTS}

This paper constitutes a summary report of research on Dasyhelea species from Poland. Many of the faunistic records presented below have already been published (KARL 1940, SZADZIEWSKI 1983, 1991, KACZOROWSKA 1996, SZADZIEWSKI 2001, SZADZIEWSKI \& BorkENT 2003, SZADZIEWSKI et al. 2004, DominiaK \& SZADZIEWSKI 2006, SZADZIEWSKI \& DOMINIAK 2006, DOMINIAK et al. 2007b, SZADZIEWSKI 2007, DOMINIAK \& SZADZIEWSKI 2008, DominiaK 2009, DominiaK \& SZADZIEWSKI 2010, GWIZDALSKAKENTZER 2011).

\section{The checklist of Polish Dasyhelea midges}

Family: Ceratopogonidae NEWMAN, 1834

Subfamily: Dasyheleinae LENZ, 1934

Genus: Dasyhelea KIEFFER, 1911

Subgenus: Dasyhelea s. str.

bilineata GOETGHEBUER, 1920

= insignipalpis KIEFFER, 1925

= saxicola (EDWARDS, 1929)

= geleiana ZILAHI-SEBESS, 1931

= montana ZILAHI-SEBESS, 1940

= lithotelmatica STRENZKE, 1950

= tecticola REMMERT, 1953

$=$ dieuzeidei (VAILlant, 1957)

= karelica GLUKHOVA \& BRODSKAYA, 1997 caesia REMM, 1993

= lugensis BRODSKAYA, 1995

flavifrons (GUÉRIN, 1833)

= obscura (WINNERTZ, 1852)

$=$ versicolor $($ WINNERTZ, 1852)

$=$ dufouri (LABOULBÈNE, 1869)

$=$ hippocastani (MıK, 1888)

= brevitibialis GOETGHEBUER, 1919

= goetghebueri KIEFFER, 1919

= lignicola KIEFFER, 1919

= sensualis KIEFFER, 1919

= paludicola KIEFFER, 1925

= oppressa THOMSEN, 1935

= septuosa BORKENT, 1997 
malleola REMM, 1962

pallidiventris (GOETGHEBUER, 1931)

= olivacea REMM, 1962

stackelbergi REMM, 1993

Subgenus: Dicryptoscena ENDERLEIN, 1936

glukhovae BRODSKAYA, 1996

lucida REMM, 1968

modesta (WINNERTZ, 1852)

= aestiva (WINNERTZ, 1852)

= longipalpis KIEFFER, 1913

= inclusa KIEFFER, 1918

= strobli KIEFFER, 1919

= pratensis GOETGHEBUER, 1920

= bihamata KIEFFER, 1923

= moascari MACFIE, 1943

= densipilosa TOKUNAGA, 1963

notata GOETGHEBUER, 1920

= semistriata GOETGHEBUER, 1921

= sziladyi ZILAHI-SEBESS, 1936

stellata REMM, 1968

thienemanni SPĂTARU \& DAMIAN-GEORGESCU, 1970

Subgenus: Prokempia KIEFFER, 1913

bilobata KIEFFER, 1915

= luteiventris GOETGHEBUER, 1934

= spiralis REMM, 1966

dampfi KIEFFER, 1925

= turfacea KIEFFER, 1925

= estonica KIEFFER, 1925

flaviventris (GOETGHEBUER, 1910)

= halobia (KIEFFER, 1924)

Subgenus: Pseudoculicoides MALLOCH, 1915

arenivaga MACFIE, 1943

auli REMM, 1962

bicrenata KIEFFER, 1923

bifida ZILAHI-SEBESS, 1936

= excellentis BORKENT, 1997

calycata REMM, 1972

corinneae GOSSERIES, 1991 


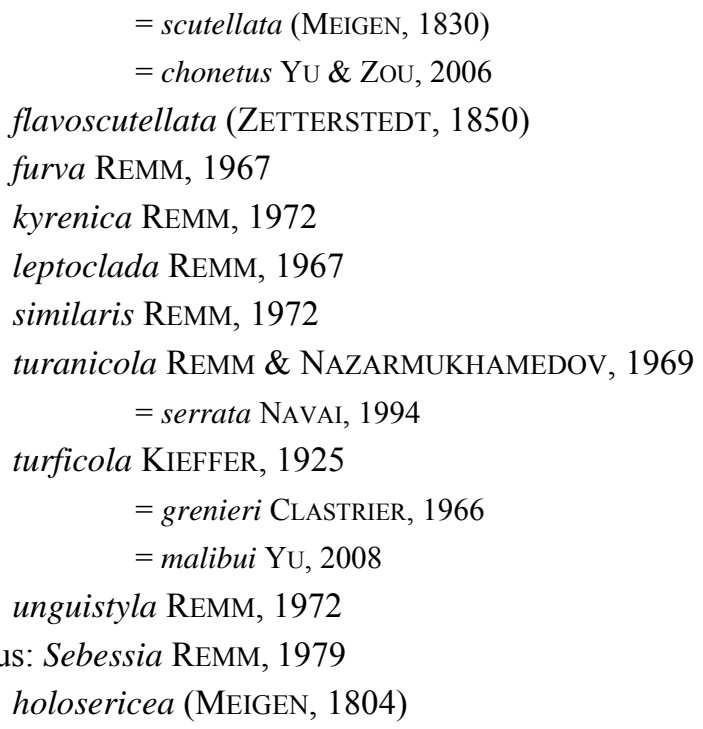

\section{Species review}

\section{Dasyhelea KIEFFER}

Dasyhelea KIEFFER, 1911: 5. Type species Dasyhelea halophila KIEFFER, 1911: 5 (by monotypy). Prokempia KIEFFER, 1913a: 163, 179. See below as subgenus.

Pseudoculicoides MALlOCH, 1915: 309. See below as subgenus.

Tetrahelea KIEFFER, 1925b: 423. Type species Culicoides insignicornis KIEFFER, 1913b: 9 (by original designation).

Dicryptoscena ENDERLEIN, 1936: 51. See below as subgenus.

Sebessia ReMm, 1979: 55. See below as subgenus.

Borkentimyia Yu et al., 2006: 321. As subgenus; systematic position unclear. Type species Dasyhelea forsteri GROGAN \& WIRTH, 1981: 97 (by original designation).

\section{Diagnosis}

Male flagellomeres sculptured. Eyes contiguous. Mouthparts similar in both sexes, mandibles and maxillae reduced, without teeth. Female palpal segment 3 without sensory pit. First radial cell usually reduced or obliterated, second radial cell short. Parameres of male genitalia usually asymmetrical. Female subgenital plate usually with fully-developed notum.

\section{Description}

Small midges, wing length 0.61-1.84 mm. Body black, dark or pale brown, rarely yellowish. Membranes between sclerites and abdominal segments pale, often yellow or grey. In some species head, scutum, postscutum, thoracic pleurites with grey, blue or 
greenish pruinose and red-brown pattern. Scutum and lateral sclerites sometimes with vitae and dark patches contrasting with background. Wing membrane transparent and colourless, only main costa and radial veins dark. Halteres milky-white, yellow, sometimes with darker knob or stem, or more rarely, entirely dark. Legs black, dark-brown, pale-brown or yellow. Femora and tibiae unicoloured, with dark bands or irregular patches. Femoro-tibial joints usually dark, tarsi pale. Head hypognathous, subspherical, slightly flattened in anteriorposterior axis. Eyes large, contiguous, reniform, with fine pubescence (Figs 13, 117, 118). Frontal sclerite connected with clypeus, elliptical, pentagonal or rhombic, sometimes with lateral processes or ribbon-like lateral border (Figs 13, 25, 26, 49, 68, 82, 112). Antenna consists of ring-like, flattened scapus, well-developed pedicel and flagellum composed of 13 flagellomeres (Figs 3, 4, 5). Male flagellomeres always sculptured (Figs 4, 5); flagellomeres 1-9 disciform, subspherical or hexagonal, with single row of long setae (sensilla chaetica), 10-12 elongate, with one or two rows of shorter setae; apex of terminal flagellomere rounded or tapered (Fig. 5), sometimes with conical or cylindrical prolongation (Fig. 23). Female flagellomeres poorly sculptured (Figs 3, 120), poorly marked reticulations present on proximal portions of all or only some flagellomeres; flagellomeres 1-8 subspherical, cylindrical or bottle-shaped, 9-12 usually more elongate; apex of 13 sometimes with conical or cylindrical prolongation (Fig. 24); all flagellomeres with single row of long setae (but shorter than in male), proximal flagellomeres with coarse sensilla trichodea. Clypeus in both sexes entire with lateral lobes (Figs 11, 50), or, more often composed of two separate sclerites (Figs 12, 27). Apex of labrum and hypopharynx with short setae, mandibles and maxillae poorly developed, vestigial, without teeth. Maxillary palp with five segments (Figs 9, 10); segments 1-2 fused; 3 usually the longest, cylindrical or slightly expanded in middle part, lacking sensory pit, but with mesal sensilla capitata (Fig. 119); 4 subcylindrical, sometimes with sensilla capitata; 5 club-shaped or elongated, subcylindrical, with one (Fig. 10) or two (Fig. 9) rows of long setae. Antepronotum with some setae, propleuron bare. Scutum prominent, with a few rows of long setae, without prescutal pit. Transverse suture well developed (Fig. 48) or reduced (Fig. 64). Paratergite long and narrow, lacking setae. Scutellum with bristles (Fig. 121). Mediotergite, as well as the rest of the body, pruinose. Anteroanepisternite D-shaped (Fig. 48). Wing narrower and longer in male than in female (Figs 6, 7); membrane with fine microtrichia (Fig. 122), macrotrichia often numerous, especially on distal portion and along wing margin; basal arculus well developed; costa short, extending to about mid-length of wing; 1st radial cell $\left(\mathrm{r}_{1}\right)$ completely obliterated or barely visible, slit-like (Figs 8, 55), 2nd radial cell $\left(\mathrm{r}_{2+3}\right)$ usually short, square, rarely obliterated; medial veins $\mathrm{M}_{1}$ and $\mathrm{M}_{2}$ forking distad of $\mathrm{r}-\mathrm{m}$ crossvein. Haltere with fine pubescence and numerous campaniform sensilla on base, as well as single sensilla on stem and knob (Fig. 123). Femora and tibiae slender; distal portions of fore tibia with two rows of coarse palisade, tibial spur absent; hind tibia 
with well-developed tibial comb (Figs 14, 124); tarsi with five tarsomeres, tarsomere 1 usually twice as long as tarsomere 2; claws small, equal-sized, slightly curved, apices bifid in male, empodium small or absent.

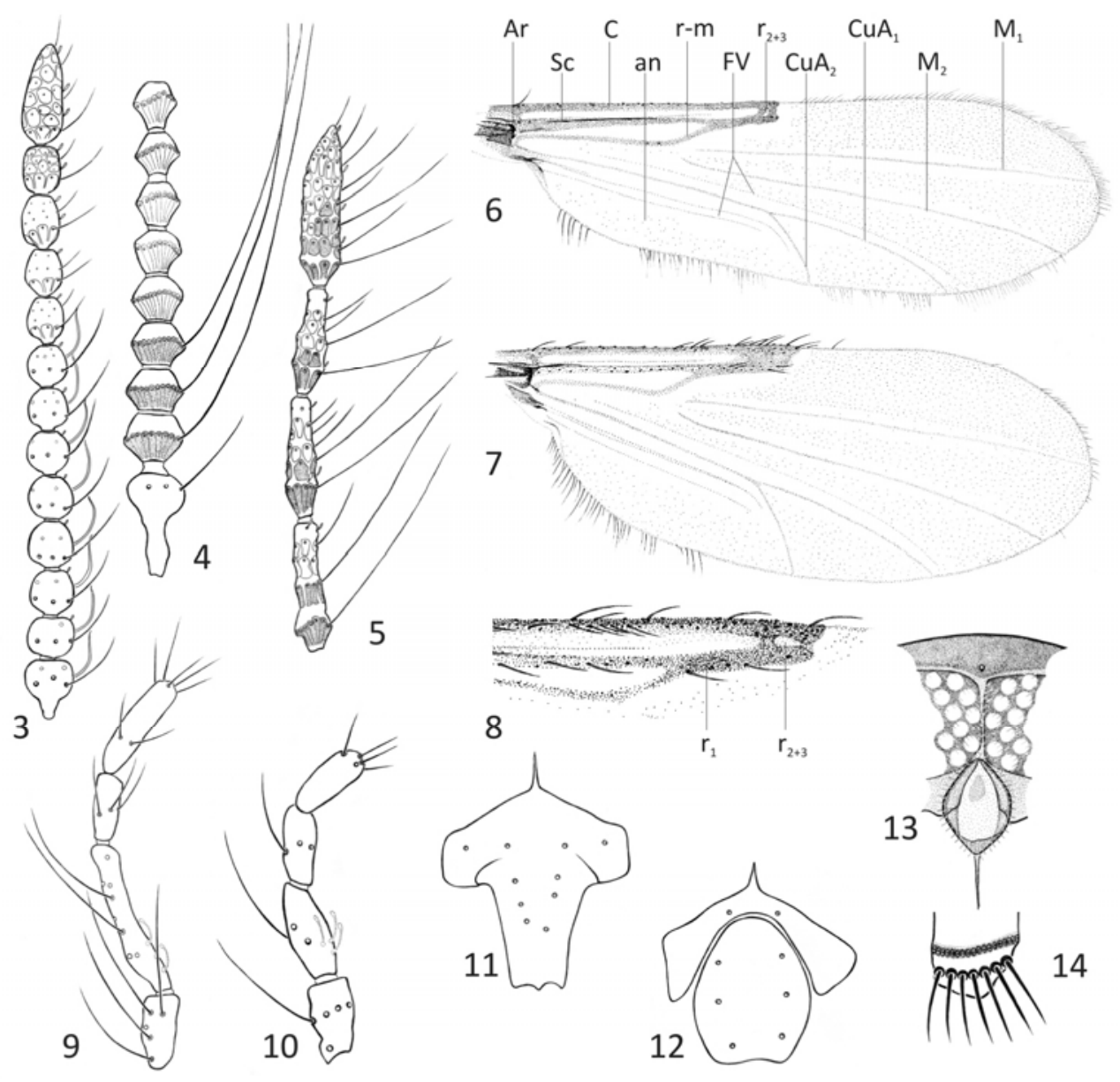

Figs 3-14. Morphology of adult male (4, 5, 6, 9, 10, 11, 14) and female (3, 7, 8, 12, 13): 3 - flagellum of Dasyhelea bilobata KIEFFER, 4 - proximal flagellomeres of D. flaviventris (GOETGHEBUER), 5 - distal flagellomeres of $D$. flaviventris (GOETGHEBUER), 6, 7 - wing of D. alonensis (STROBL), 8 - radial cells of $D$. mayor (STROBL), 9 - palpus of D. modesta (WINNERTZ), 10 - palpus of D. bilobata KIEFFER, 11 - clypeus of D. modesta (WINNERTZ), 12 - clypeus of D. bilobata KIEFFER, 13 - eyes and frontal sclerite of D. bilobata KIEFFER, 14 - tibial comb of hind leg of $D$. modesta (WINNERTZ).

Abbreviations: $\mathrm{Ar}$ - arculus, an - anal cell, $\mathrm{C}$ - costa, $\mathrm{CuA}_{1}, \mathrm{CuA}_{2}$ - cubital veins, $\mathrm{FV}$ - false veins, $\mathrm{M}_{1}, \mathrm{M}_{2}$ - medial veins, $\mathrm{r}_{1}$ - first radial cell, $\mathrm{r}_{2+3}$ - second radial cell, $\mathrm{r}-\mathrm{m}$ - radial-medial crossvein, $\mathrm{Sc}-$ subcosta. 

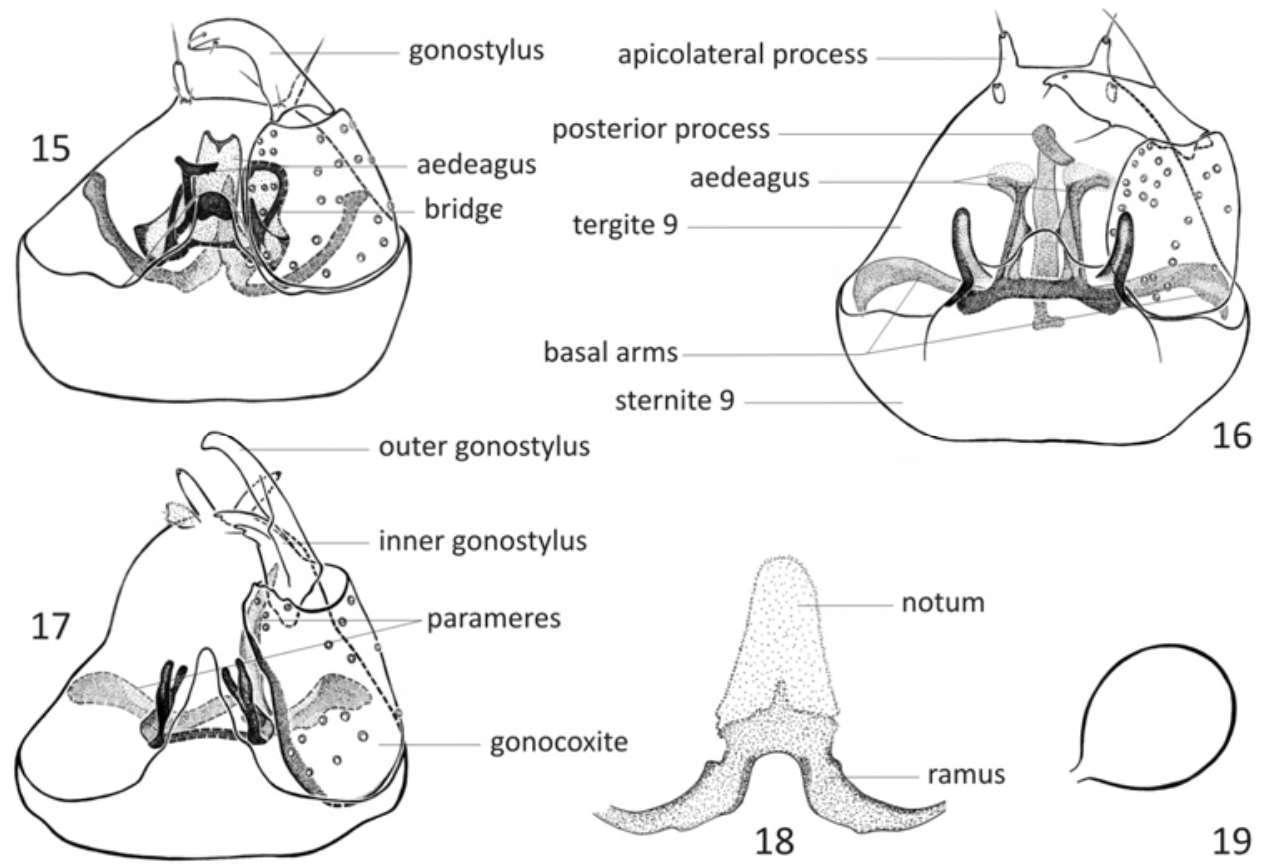

Figs 15-19. Male $(15,16,17)$ and female $(18,19)$ genitalia: 15 - Dasyhelea turanicola Remm \& NAZARmukhamedov, 16 - D. kyrenica Remm, 17 - D. furva Remm, 18, 19 - D. bilobata KiefFer.

Male genitalia (Figs 15, 16, 17). Tergite and sternite of 9th abdominal segment fused. Tergite 9 tapering posteriorly, with blunt or rounded apex and apicolateral processes. Sternite 9 short, posterior margin with shallow excavation, straight or extended - conical, rhomboidal or bifid. Gonocoxite conical or cylindrical. Gonostylus of various shape and size, single (Figs 15, 16) or rarely divided into two parts (Fig. 17). Depending on species, parameres symmetrical, asymmetrical, either divided or fused medially. Aedeagus in the shape of flat or arched plate, with additional median, submedian or lateral projections of various sizes and shapes. Bridge joining aedeagus with sternite 9, well visible if present (Fig. 15).

Female genitalia. Gonapophyses of sternite 9 form subgenital plate, composed of notum and ramus (Fig. 18). Tergite and sternite of abdominal segment 10 fused, with pair of short cerci originating from the vestigial 11th segment. Spermatheca (Fig. 19) usually single, with short neck, elliptical, ovoid, spherical or retort-shaped.

\section{Key to subgenera of adult Dasyhelea}

1. Male genitalia with an envelope covering entire aedeagus (Fig. 114); parameres symmetrical. Two functional spermathecae present (Fig. 115) 
-. Male genitalia without an envelope covering entire aedeagus; parameres symmetrical or asymmetrical. Spermatheca single ..... .2

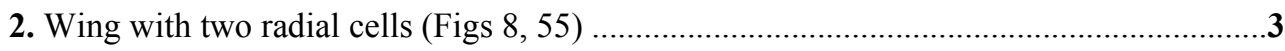

-. Wing with one radial cell ...........................................................................................4

3. Clypeus entire with lateral lobes (Figs 11, 50). Posterior margin of male sternite 9 extended, with divided apex (Fig. 56, 59, 60, 61, 62, 63); apicolateral processes of tergite 9 small

Dicryptoscena

-. Clypeus divided into separate sclerites (Fig. 12), or if entire with lateral lobes then wing with one radial cell. Posterior margin of male sternite 9 without divided apex; apicolateral processes of tergite 9 usually prominent

Pseudoculicoides (in part)

4. Frontal sclerite narrow, elongate, elliptical or slightly club-shaped, evenly sclerotized (Figs 82, 83). Posterior margin of male sternite 9 extended, without divided apex

Pseudoculicoides (in part)

-. Frontal sclerite broad, or if narrower then with lateral borders and distinctly sclerotized in mid-portion (Figs 13, 25, 26, 67, 68). Posterior margin of male sternite 9 otherwise .5

5. Frontal sclerite broader than long, elliptical, pentagonal or slightly heart-shaped, with long slender ventral projection (Fig. 25, 26). Posterior margin of male sternite 9 straight, arch-like or slightly concave (Fig. 37, 46, 47). Female antennal flagellomeres elongate, bottle-shaped, with sculptured reticulations; flagellomere 13 with apical prolongation (Fig. 24)

Dasyhelea s. str.

-. Frontal sclerite longer than broad, ovoid, with ribbon-like lateral borders, distinctly sclerotized in mid-portion (Fig. 13, 67, 68). Posterior margin of male sternite 9 with pincer-like median process (Fig. 74, 77, 78). Female antennal flagellomeres short, spherical, only some are slightly reticulated; flagellomere 13 without apical prolongation (Figs 3, 70)

Prokempia

\section{subgenus Dasyhelea}

\section{Diagnosis}

Frontal sclerite broad, elliptical, pentagonal or slightly heart-shaped, with long, slender projection. Antennal segments sculptured. Apex of last flagellomere with cylindrical prolongation. Thorax with greyish, bluish or greenish pollinose, and usually with brownishred pattern on scutum. Wing with one radial cell.

Male. Apicolateral processes of tergite 9 prominent. Posterior margin of sternite 9 shallow concave, straight or low arched. Gonocoxite often with mesoventral hook. Gonostylus long, nearly straight or slightly curved. Parameres asymmetrical.

Female. All antennal segment elongate, in most species bottle-shaped. Subgenital plate prominent, notum usually triangular, but sometimes arch-shaped with irregular or rounded 


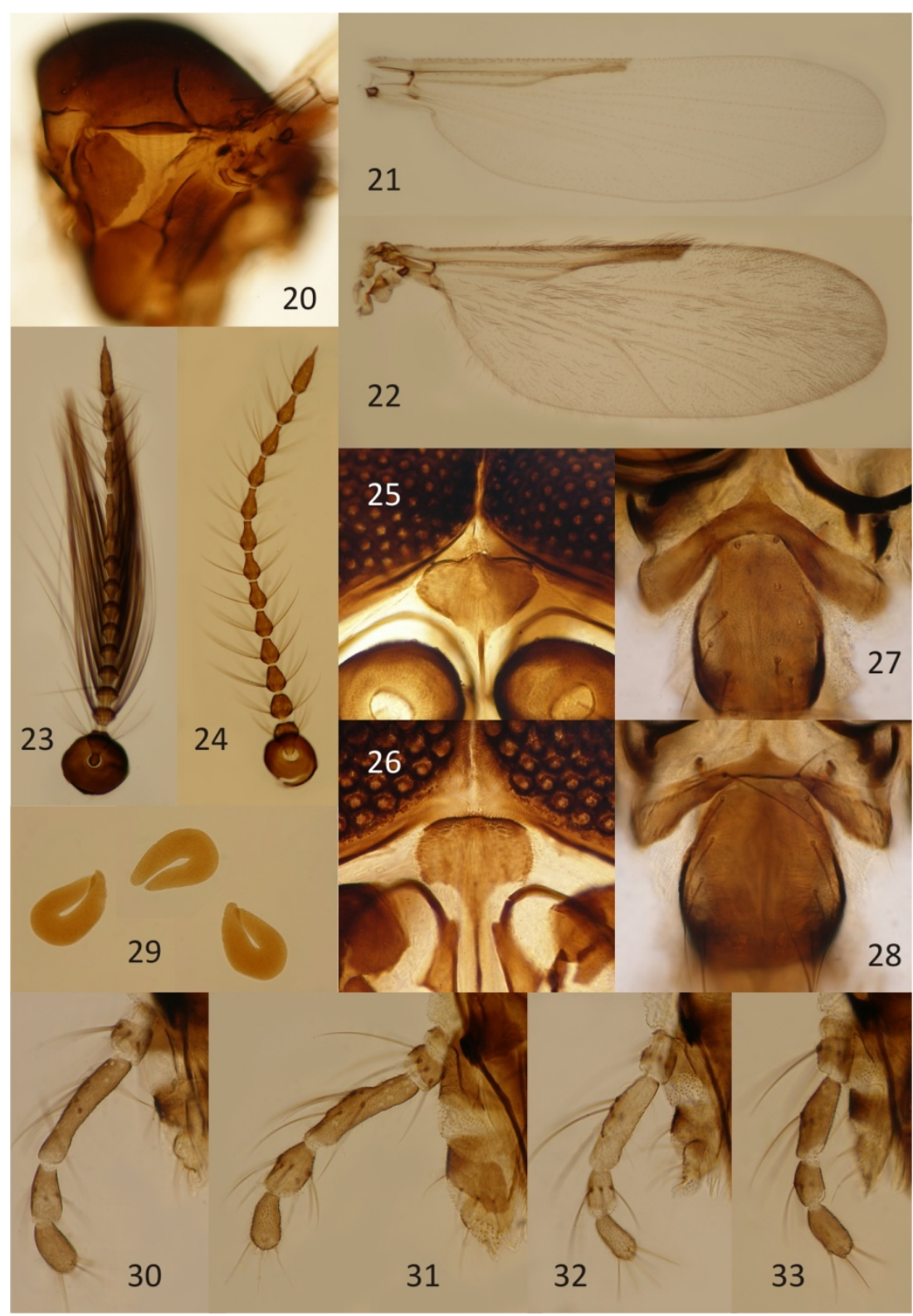

Figs 20-33. Dasyhelea bilineata Goetghebuer, male (23, 28, 30), female (22, 24, 25, 29, 31), D. flavifrons (GUÉRIN), male (32), female (33), D. caesia REMM, male (21, 27), D. malleola REMM, female (20), D. pallidiventris (GOETGHEBUER), female (26).

20 - thorax, 21, 22 - wing, 23, 24 - antenna, 25, 26 - frontal sclerite, 27, 28 - clypeus, 29 - eggs, $30,31,32,33$ - palpus. 
anterior projection. Spermatheca single, pyriform, retort-shaped, rarely subspherical, with short neck.

\section{Discussion}

The subgenus Dasyhelea is identical to the grisea group sensu WIRTH (1952) and WAUGH \& WIRTH (1976). Two groups of species within this subgenus can be distinguished by differences in the shape of their pupal respiratory horns and posterior processes of the anal segment, and male parameres. The first of these include most species in the subgenus Dasyhelea, the known pupae of which have flattened, straight to slightly curved respiratory horns (Fig. 126) with spiracles that are primarily on the apex, and sparse lateral spiracles (Fig. 127). The pupal anal segment bears short, divergent lateral tubercles (Fig. 128). The bases of male parameres are inclined and slightly deflected and the posterior process is wavy or C-shaped (Figs 34, 37, 40, 43), and often fused to the right basal arm. The second group includes three Nearctic species: D. chani WIRTH \& LINLEY, D. pollinosa WIRTH and D. traverae THOMSEN; a Neotropical species: D. paulistana FoRATTINI \& RABELLO; three Palaearctic species: D. caesia REMM, D. stackelbergi REMM and D. unicolor REMM; a species described from Sumatra (as Holoconops sp., MAYER 1934c: 201). Pupae are known only for D. chani, D. caesia (see description of D. lugensis BRODSKAYA), D. paulistana, D. pollinosa, D. traverae and the Sumatran species, as all have sharp-pointed respiratory horns, with spiracles located laterally and the posterior processes of the anal segment are very long, slender and not divergent. The male parameres are usually divided, their bases are not deflected and are situated in the middle of the hypopygium, and the posterior process is nearly straight, and perpendicular to the basal arms (Figs 46, 47).

Six species in the subgenus Dasyhelea have been reported from Poland. In Europe, there are four other species that have not yet been found in Poland: D. baltica REMM, D. bensoni EDWARDS, D. incisurata REMM and D. unicolor REMM.

\section{Key to species}

1. Bases of parameres inclined, slightly deflected; posterior process wavy or C-shaped (Figs 34, 37, 40, 43). Female subgenital plate prominent; notum usually triangular

-. Bases of parameres not deflected, situated in the middle of hypopygium; posterior process straight (Figs 46, 47). Female subgenital plate fine, arch-shaped, sometimes with irregular or rounded anterior projection

2. Palpal segments 3 and 4 with sensilla capitata .pallidiventris

-. Palpal segment 3 with sensilla capitata

3. Apex of gonostylus broad (Fig. 37). Female subgenital plate with leaf-shaped notum (Fig. 39) malleola -. Apex of gonostylus slender. Female subgenital plate with triangular notum (Figs 42, 45) 
4. Male palpal ratio $\mathrm{PR}_{\text {(III) }}$ greater than 4.0. Female subgenital plate elongate; notum in the shape of isosceles triangle, usually weakly sclerotized at base (Fig. 42)

bilineata

-. Male palpal ratio $\mathrm{PR}_{\text {(III) }}$ less than 4.0. Female subgenital plate short; notum in the shape of equilateral triangle, evenly sclerotized (Fig. 45)

flavifrons

5. Submedian projections of aedeagus slender, wavy; posterior margin of sternite 9 extended, with short preapical setae (Fig. 46)

caesia

-. Submedian projections of aedeagus broad, lobate; posterior margin of sternite 9 with shallow excavation, lacking preapical setae (Fig. 47)

stackelbergi

\section{Dasyhelea pallidiventris (GOETGHEBUER, 1931)}

(Figs 26, 34, 35, 36)

Tetraphora pallidiventris GoETGHEBUER, 1931: 211 ((Dasyhelea), description and figure - female, Germany).

Dasyhelea pallidiventris: SzADZIEWSKI 1986: 43 (description and figure - male, female, syn.: = olivacea, Germany, Poland); TÓTHOvÁ et al. 2004b: 146 (Czech Republic); DominiAK \& SZADZIEWSKI 2006: 5 (Poland); DominiAK \& SZADZIEWSKI 2010: 24 (syn.: = olivacea, = versicolor sensu REMM 1979, comments on biology, Finland, North Korea); GwizDALSKAKENTZER 2011: 37 (Poland).

Dasyhelea olivacea REMM, 1962: 117 (description and figure - male, female, Estonia); REMM 1966: 58 (Lithuania); REMm 1967: 16 (Azerbaijan, Georgia); REMM \& ZHoGOLEV 1968: 832 (Ukraine: Crimea); SzADZIEWSKI 1983: 66 (Poland).

Dasyhelea versicolor: REMM 1979: 50 (syn.: = olivacea, Estonia). Nec D. versicolor (WINNERTZ, 1852).

\section{Diagnosis}

Sensilla capitata present on palpal segments 3 and 4. Gonostylus stout, with rounded apex. Female subgenital plate hastate, notum often weakly sclerotized.

\section{Description}

Male. AR 0.85-0.96 $(\mathrm{n}=12)$. Palpal segment 3 elongate, 59-75 $\mu \mathrm{m}$ long $(\mathrm{n}=14), \mathrm{PR}_{\text {(III) }}$ 2.95-3.76 $(\mathrm{n}=13)$; sensilla capitata present on segments 3 and 4. Scutellum yellow or tawny, with 6-9 bristles and some smaller setae. Wing length 1.19-1.49 $\mathrm{mm}(\mathrm{n}=14)$, CR 0.48-0.50 $(\mathrm{n}=14)$. Legs unicoloured or with irregular, dark patches on femora and tibiae. Genitalia (Fig. 34). Posterior margin of sternite 9 arched. Gonocoxite bearing prominent mesoventral hook. Gonostylus rather stocky, with rounded apex. Parameres asymmetrical; posterior process broadly fused to right basal arm. Anterior margin of aedeagus low arched or straight, with a pair of submedian, slightly conical projections.

Female. AR 0.77-0.89 $(n=13)$. Length of 3rd palpal segment 49-66 $\mu \mathrm{m}(\mathrm{n}=12) ; \mathrm{PR}_{(\mathrm{III})}$ 2.48-3.37 $(\mathrm{n}=12)$; sensilla capitata present on segments 3 and 4. Scutellum entirely yellow 
or infuscated on mid-portion, provided with ca 9 bristles and a few smaller setae. Wing length 1.06-1.22 mm $(\mathrm{n}=12)$; CR 0.49-0.53 $(\mathrm{n}=12)$. Coloration of the legs as in male. Genitalia (Figs 35, 36). Notum in subgenital plate hastate, often with weakly sclerotized lateral margins. Spermatheca pyriform or retort-shaped; length 72-88 $\mu \mathrm{m}(\mathrm{n}=13)$, width $46-55 \mu \mathrm{m}(\mathrm{n}=12)$.
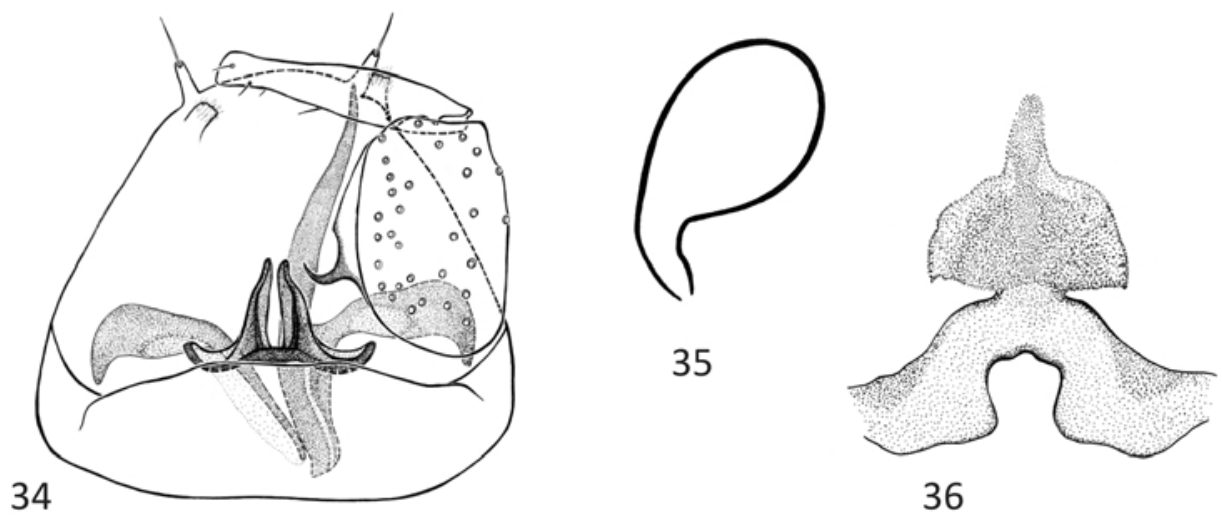

Figs 34-36. Dasyhelea pallidiventris (Goetghebuer): 34 - male genitalia, 35 - spermatheca, 36 - female subgenital plate.

\section{Material examined $(n=63)$}

Poland: Southern Baltic CoAstlands. Gdańsk, Sobieszewska Is., Górki Wschodnie, 27 June 1970, 1 male, leg. B.S.; 3 July 1970, 1 female, leg. B.S.; 3 August 1970, 1 male, leg. B.S.; 4 August 1970, 1 male, leg. B.S.; 5 August 1970, 1 male, leg. B.S.; saline soil, 5 May 1975, ex cult., 1 male, 1 female, leg. R.Sz.; saline soil, 10 June 1977, 1 female, leg. R.Sz.; 18 July 1977, 2 females, leg. R.Sz.; 18 August 1977, 1 female, leg. R.Sz.; saline habitat, 18 August 1977, 1 male, 1 female, leg. R.Sz.; beach, 20 August 2000, net, 2 males, 1 female, leg. E.K.; 29 July 2004, net, 1 female, leg. E.K.; 12 July 2005, net, 2 males, 1 female, leg. E.K. Krzewsk near Elbląg, Lake Drużno, 10 July 1983, 2 males, leg. R.Sz. Mechelinki near Gdynia, beach, sun, $22^{\circ} \mathrm{C}, 27$ August 2006, net, 1 male, 1 female, leg. K.B. Żarnowiec, mud from lake, 10 May 1984, ex cult., 1 male, leg. R.Sz. EASTERN Baltic CoAstLands. Barciany near Kętrzyn, near pond, 12 July 1981, 1 female, leg. R.Sz. Łęknica near Kętrzyn, forest margin, 16 July 1981, 1 male, leg. R.Sz. SouthERn Baltic LaKELANDS. Gdynia Bernadowo, Lęg nad Swelinią Reserve, 27 August 2011, light trap, 1 male, leg. M.G.K.; 28 August 2011, light trap, 1 male, leg. M.G.K. Ocypel near Starogard Gdański, Pimpinella saxifraga L., 31 July 1984, 1 male, leg. J.K. Szubin near Bydgoszcz, 26 July 1993, 2 males, leg. J.K. Inowrocław Mątwy, saline habitat, 15 May 1975, 1 female, leg. R.Sz. EASTERn BAltic LAKELANDS. Kętrzyn, near pond, Apiaceae, 22 July 1981, 2 males, 2 females, leg. R.Sz. Mazurki near Augustów, peat bog forest, 15 May 2005, net, 1 male, leg. P.D. Olsztyn, Apiaceae, 29 July 1990, 1 female, leg. K.P.; 11 August 1990, 1 male, leg. K.P. Piecki near Mragowo, Lake Wagiel, 5 August 1988, 1 female, leg. R.Sz. Płaska near Augustów, 16 August 2007, net, 1 male, leg. P.D. Orłowo near Olecko, on Lake 
Litygajno, Apiaceae, 24 July 2007, net, 1 male, leg. A.B. Silec near Kętrzyn, forest, 2 May 1980, 2 females, leg. R.Sz.; wet-ground forest, 5 July 1981, 2 males, 1 female, leg. R.Sz.; lake, 23 July 1981, 1 male, leg. R.Sz. Solanka near Kętrzyn, near lake, Apiaceae, 15 August 1980, 1 female, leg. R.Sz. Słupie near Suwałki, near the River Czarna Hańcza, peat bog, 5 July 2005, net, 1 male, leg. P.D. PODLASIE. Hamlet of Maruszka on Siemianówka Reservoir near Białystok, Apiaceae, 11 August 2005, net, 1 male, leg. P.D. Kunicha near Augustów, Angelica sylvestris L., 24 August 1984, 1 male, 1 female, leg. J.K.; Anethum graveolens L., 27 August 1984, 1 female, leg. J.K.; Cicuta virosa L., 18 August 1985, 1 male, leg. J.K.; Pimpinella sp., 21 August 1985, 1 female, leg. J.K. Sztabin near Augustów, Peucedanum palustre (L.) MoEnCH, 20 August 1984, 1 male, leg. J.K.; Angelica sylvestris L., 24 August 1985, 1 male, leg. J.K. Central Poland Lowlands. Puszcza Biała, 2 July 1986, 1 male, IZ PAN. SudeTy MTs. Radomierz near Jelenia Góra, Trzcińskie Mokradła, 25 June 1993, 1 male, leg. E.K. Pokrzywna near Głuchołazy, 11 August 1983, net, 1 male, leg. R.Sz. CENTRAL MAŁOPOLSKA UPLAND. Święta Katarzyna near Kielce, meadow on the River Czarna Woda, $50^{\circ} 55.158^{\prime} \mathrm{N} 20^{\circ} 54.136$ 'E, 276 m AMSL, 27 July 2008, net, 1 female, leg. P.D.

\section{Distribution and biology}

Sweden, Finland, Estonia, Lithuania, Germany, Poland, Czech Republic, Ukraine (Crimea), Georgia, Azerbaijan, North Korea (DOMINIAK \& SZADZIEWSKI 2010).

Larvae live in inland saline habitats and the littoral zone of lakes. SZADZIEWSKI (1983) identified $D$. pallidiventris as haloxene. Adults of this species very often visit umbelliferous flowers.

In Poland D. pallidiventris is known from numerous localities. Adult flight period MayAugust (Remm 1962, 1966, 1967, Remm \& ZHogolev 1968, ReMm 1979, Tóthová et al. 2004b, DOMINIAK \& SZADZIEWSKI 2010, GWIZDALSKA-KENTZER 2011, unpublished data).

\section{Discussion}

Among the Eurasian species of the subgenus Dasyhelea only D. pallidiventris and D. bensoni EDWARDS have sensilla capitata on both palpal segments 3 and 4. Dasyhelea bensoni is close to $D$. pallidiventris, but is much darker, has a markedly sclerotized notum in the female subgenital plate, and usually well visible labia.

The holotype female of $D$. pallidiventris is deposited at the Royal Belgian Institute of Natural Sciences in Brussels (SZADZIEWSKI 1986).

\section{Dasyhelea malleola REMM, 1962}

(Figs 20, 37, 38, 39)

Dasyhelea malleolus REMM, 1962: 119 (description - male, female, figure - male, Estonia); HAVELKA 1976a: 75 (Germany); HAVELKA 1976b: 226 (description and figure - male); REMM 1979: 50 (Estonia).

Dasyhelea malleola: DelÉColle 2002: 28 (Andorra); SZADZIEWSKI et al. 2004: 120 (Algeria, Poland); TóthovÁ et al. 2004b: 146 (Czech Republic); DominiaK \& SzADZIEWSKi 2010: 19 (Spain, Ukraine). 


\section{Diagnosis}

Apex of gonostylus broad, bulbous. Subgenital plate elongate, with leaf-shaped notum.

\section{Description.}

Male. AR 0.80-0.87 ( $\mathrm{n}=7)$. Palpal segment 3 short, cylindrical, 38-47 $\mu \mathrm{m}$ long $(\mathrm{n}=6)$, $\mathrm{PR}_{(\mathrm{III})}$ 2.62-3.36 $(\mathrm{n}=6)$. Scutellum yellow or dark, with paler margins, bearing 6 bristles. Wing length 0.87-1.03 mm $(n=5)$, CR 0.48-0.50 $(n=5)$. Legs dark. Genitalia (Fig. 37). Posterior margin of sternite 9 arched. Apex of gonostylus very broad, bulbous. Parameres asymmetrical, posterior process distinctly fused to right basal arm. Aedeagus with anterior margin low arched, bearing two submedian projections.

Female. AR 0.74-0.76 $(\mathrm{n}=3)$. Length of third palpal segment 41-43 $\mu \mathrm{m}(\mathrm{n}=3), \mathrm{PR}_{(\mathrm{III})}$ 2.56-2.87 $(\mathrm{n}=3)$. Scutellum yellow or dark, with paler margins, armed with 6 or 7 bristles. Wing length 0.97-1.04 mm $(\mathrm{n}=2)$, CR 0.50-0.51 $(\mathrm{n}=2)$. Legs unicoloured. Genitalia (Figs $38,39)$. Subgenital plate elongate, notum leaf-shaped. Spermatheca with short neck, retortshaped, 80-100 $\mu \mathrm{m}$ long $(\mathrm{n}=3)$ and 47-56 $\mu \mathrm{m}$ wide $(\mathrm{n}=3)$.

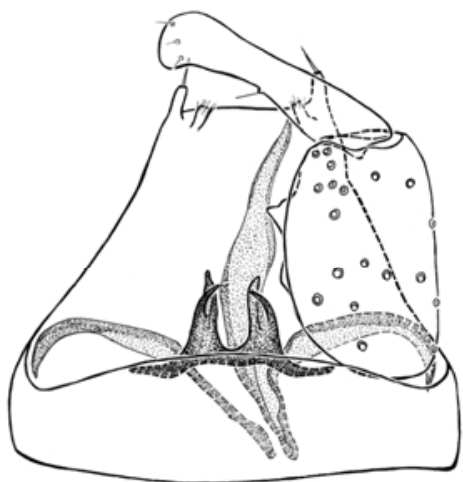

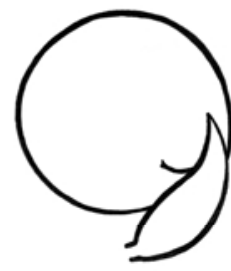

38

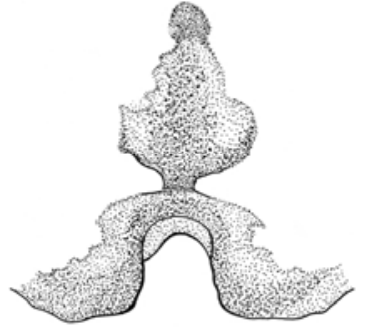

39

\section{7}

Figs 37-39. Dasyhelea malleola Remm: 37 - male genitalia, 38 - spermatheca, 39 - female subgenital plate.

Material examined $(n=8)$

Poland: EASTERn BALtic LaKelands. Słupie near Suwałki, near the River Czarna Hańcza, peat bog, 24 May 2006, net, 2 males, leg. P.D. Central Western CARPATHIAns. Dolina Małej Łąki in the Tatra Mts., 11 July 2003, net, 1 male, leg. M.G.K. Szafranówka in the Pieniny Mts., 24 June 2003, net, 1 male, leg. M.G.K. EASTERn BESKID MTS. Ustrzyki Górne, 23-30 July 1980, 1 female, leg. R.Sz.

Other specimens

Ukraine: Alupka, garden at Vorontsov's Moorish Castle, 21 May 2008, net, 1 female, leg. P.D. Spain: Maitena, Granada Prov., 11 July 1960 - 900 m., J.R. Vockeroth, 2 females, [USNM]. 


\section{Distribution and biology}

Estonia, Germany, Poland, Czech Republic, Ukraine (Crimea), Spain, Andorra, Algeria (DOMINIAK \& SZADZIEWSKI 2010).

Up to now $D$. malleola has been reported from the north-eastern and southern regions of Poland. In Europe adults fly from May until the end of July (REMM 1962, HAVELKA 1976b, REMM 1979, TótHOVÁ et al. 2004b, DOMINIAK \& SZADZIEWSKI 2010).

\section{Discussion}

The original description of the female (REMm 1962) is inadequate. Currently, four female specimens were found in the materials examined. Except for the usual sexual differences, they are similar to males of D. malleola. Among the remaining European species of the subgenus, females of $D$. malleola are readily distinguished by the shape of the subgenital plate. D. stemlerae WAUGH \& WIRTH, known from the Nearctic Region, has similar female genitalia.

The first information about the occurrence of D. malleola in Spain (REMM 1988), subsequently reiterated by DELÉCOLLE (2002), should be treated as a doubtful record.

\section{Dasyhelea bilineata GOETGHEBUER, 1920}

(Figs 22, 23, 24, 25, 28, 29, 30, 31, 40, 41, 42, 126, 127, 128)

Dasyhelea bilineata GoEtGHeBuER, 1920: 45 (description, in key - male, Belgium); GOETGHEBUER 1925: 121 (description and figure - larva, pupa, male, female, breeding site - leaf axils of Common Teasel); THIENEMANn 1925: 105, 107 (in key - larva, pupa); MünCHBERG 1955: 390 (breeding site - leaf axils of Common Teasel, comments, Germany); DiSNEY \& WIRTH 1982: 233 (breeding site - leaf axils of Common Teasel, Great Britain); DOMINIAK \& SZADZIEWSKI 2010: 7 (syn.: = insignipalpis, $=$ saxicola,$=$ geleiana,$=$ montana, $=$ lithotelmatica,$=$ tecticola, = dieuzeidei, $=$ karelica, breeding site - leaf axils of Dipsacus sp., Spain, Norway, Romania, Slovakia, Turkey, Ukraine); GwIZDALSKA-KENTZER 2011: 37 (Poland).

Culicoides versicolor: MUNSTERHJELM 1920: 160 (description and figure - egg, egg mass, breeding site - rock pools, Finland). Nec D. versicolor (WINNERTZ, 1852).

Dasyhelea insignipalpis KIEFFER, 1925c: 62 (as D. versicolor var. insignipalpis, description and figure - male, female, breeding site - stoup, Germany).

Prokempia bilineata: KIEFFER 1925c: 72 (description - male).

Tetraphora saxicola EDWARDS, 1929: 426 (description - male, female, breeding site - rock-holes, Great Britain).

Dasyhelea geleiana ZILAHI-SEBESS, 1931: 321 (description and figure - larva, pupa, male, female, breeding site - rock pools, biology, Hungary); ZILAHI-SEBESS 1936b: 199 (Hungary); ZILAHISEBESS 1940: 48 (description, figure - male, as D. dufouri var. geleiana, syn.: = bilineata, = geleiana, = saxicola?, = sensualis, Slovakia, Hungary); VALKANOV 1941: 201 (breeding site - rock pools, comments on biology, Bulgaria); OHM \& REMMERT 1955: 204 (description and figure - pupa, breeding site - rock pools, France).

Dasyhelea saxicola: EDWARDS 1933: 91 (figure - male, taxonomic comments); ZAVŘEL 1935: 1 ((Tetraphora), breeding site - rock-holes, France); SZADZIEWSKI 1986: 47 (description and 
figure - male, female, syn.: = lithotelmatica, breeding site - rock pools, Austria, Great Britain); KNOZ 1997: 81 (Czech Republic); KNOZ 1998: 118 (Czech Republic); TótHOvÁ et al. 2004b: 146 (Czech Republic); SzADZIEWSKI \& DOMINIAK 2006: 143 (description and figure - male, female, syn.: = lithotelmatica, $=$ geleiana,$=$ tecticola,$=$ karelica, breeding site - sink, rain gutter, rockholes, rock pool, float system in greenhouse, Algeria, France, Germany, Poland, Great Britain, Italy); AsHe et al. 2007: 115 (syn.: = lithotelmatica, = geleiana, = tecticola, = karelica, = montana, breeding site - rock pools, rock-holes, biology, Ireland, Great Britain).

Dasyhelea obscura: ZAVŘEL 1935: 2 (as Dasyhelea obscura GOETG., breeding site - rock pools, Croatia); STORÅ 1939: 17 (breeding site - rock pools, Finland); REMM 1962: 113 (description - male, female, figure - male, Estonia). Nec D. obscura (WINNERTZ, 1852).

Dasyhelea montana ZILAHI-SEBESS, 1940: 48 (description, as D. dufouri var. montana, syn.: = bilineata, $=$ geleiana,$=$ saxicola, , = sensualis, Slovakia, Hungary).

Dasyhelea lithotelmatica STRENZKE in THIENEMANN 1950: 178 (description and figure - larva, pupa, male, female, breeding site - rock pools, Austria); THIENEMANN 1950: 167 (breeding site - rock pools, Austria); TUZET \& ORMIÉRES 1964: 163 (breeding site - rainwater pools, France); DiSNEY 1975: 227 (breeding site - rock pools, Great Britain).

Dasyhelea tecticola REMMERT, 1953: 334 (description - larva, pupa, male, female, figure - pupa, male, female, breeding site - rain gutters, Germany); SANNINO \& ESPINOSA 2004: 32 (description - larva, pupa, male, female, figure - larva, pupa, breeding site - float system in greenhouse, Italy).

Culicoides dieuzeidei Vaillant, 1957: 265 (description and figure - male, female, breeding site - rock pools, France, Algeria).

Dasyhelea karelica GLUKHOVA \& BRODSKAYA, 1997: 443 (description and figure - larva, pupa, male, female, breeding site - rock-hole, Russia: Karelia).

Dasyhelea dufouri: SzADZIEwsKi 1998: 103 (Switzerland). Nec D. dufouri (LABOUlbèNE, 1869).

\section{Diagnosis}

Wing length of male 1.6-1.8 mm, of female 1.5-1.8 mm. Third palpal segment slender in both sexes. Male palpal ratio $\mathrm{PR}_{(\text {(III) }}$ more than 4.0. Female subgenital plate elongate, notum in shape of isosceles triangle, in basal part weakly sclerotized. Femora and tibiae of all legs usually with dark bands.

\section{Description}

Male. AR 0.93-1.04 $(\mathrm{n}=11)$. Palpal segment 3 cylindrical, slender (Fig. 30); length 85-101 $\mu \mathrm{m}(\mathrm{n}=12), \mathrm{PR}_{(\mathrm{III})} 4.14-4.94(\mathrm{n}=12)$. Scutellum pale or dark, with 10-12 bristles and some smaller setae. Wing length 1.57-1.84 mm $(\mathrm{n}=12)$, CR 0.50-0.54 $(\mathrm{n}=12)$. Femora and tibiae with distinct bands or whole dark. Genitalia (Fig. 40). Posterior margin of sternite 9 low, arch-shaped. Gonocoxite usually with conspicuous mesoventral hook. Gonostylus slender, evenly curved, C-shaped. Parameres asymmetrical. Posterior process and right basal arm very often fused. Anterior margin of aedeagus low-arched or nearly straight. Two submedian, posteriorly directed projections of aedeagus present.

Female. AR 0.76-0.86 $(\mathrm{n}=15)$. Palpal segment 3 cylindrical, slightly expanded in the middle (Fig. 31), 75-88 $\mu \mathrm{m}$ long $(\mathrm{n}=15), \mathrm{PR}_{\text {(III) }} 3.07-4.00(\mathrm{n}=14)$. Scutellum yellow, with 
10-14 bristles and numerous setae. Wing with one radial cell (Fig. 22); length 1.49-1.75 $\mathrm{mm}(\mathrm{n}=15)$, CR 0.52-0.57 $(\mathrm{n}=15)$. Colour of the legs the same as in male. Genitalia (Figs $41,42)$. Subgenital plate elongate, notum in shape of isosceles triangle, weakly sclerotized in the basal part. Spermatheca with short neck, retort-shaped, dimensions 84-104 $\mu \mathrm{m}(\mathrm{n}=$ 11) $\times 57-67 \mu \mathrm{m}(\mathrm{n}=11)$.
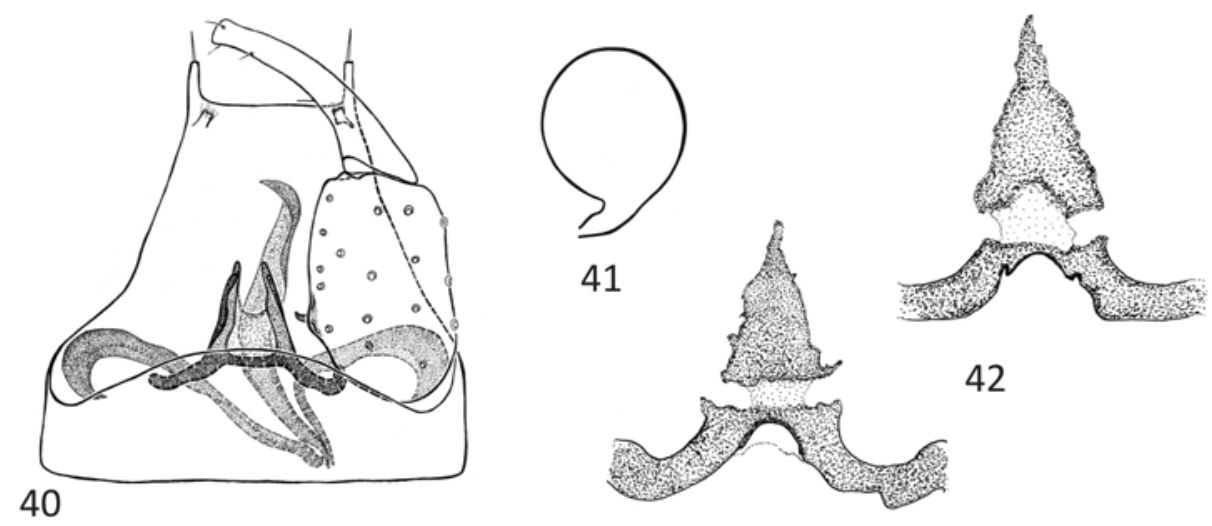

Figs 40-42. Dasyhelea bilineata Goetghebuer: 40 - male genitalia, 41 - spermatheca, 42 - female subgenital plate.

Material examined $(n=128)$

Poland: Southern Baltic Coastlands. Elbląg, greenhouse, 8 June 1982, 1 male, leg. R.Sz. Gdańsk, 8 July 1980, 2 females, leg. R.Sz.; gutter, 8-20 July 1980, ex cult., 8 females, leg. R.Sz.; 8 July -20 August 1980, ex cult., 1 pupa, 3 males, 1 female, leg. R.Sz.; sink in a garden, 7 July 1979, ex cult., 1 female, leg. R.Sz.; 7 October 1979, 4 larvae, leg. R.Sz.; 7 October 1979, ex cult., eggs, 15 October 1979, 1 male, 1 female, leg. R.Sz.; 8 July 1980, ex cult., 18 pupae, 4 males, 12 females, leg. R.Sz.; 20-26 June 1982, ex cult., 3 pupae, 2 males, 2 females, leg. R.Sz.; dish near tap, 7 October 1979, ex cult., 5 males, leg. R.Sz. Gdańsk Brzeźno, beach, 11 June 2000, net, 1 male, leg. E.K. Gdańsk Jelitkowo, beach, 8 July 2000, net, 1 male, 1 female, leg. E.K. Gdynia Orłowo, beach, 28 August 1999, net, 1 female, leg. E.K. Gdynia Wzgórze Św. Maksymiliana, beach, 15 September 2000, net, 1 female, leg. E.K. Mechelinki near Gdynia, beach, sun, $22^{\circ} \mathrm{C}, 27$ August 2006, net, 1 female, leg. K.B. Sopot - Gdańsk Jelitkowo, beach, 6 August 2000, net, 2 males, leg. E.K. Puck, beach, 8 August 2000, net, 1 male, 1 female, leg. E.K.; 28 September 2000, net, 2 females, leg. E.K. Sopot, beach, 28 August 1999, net, 1 male, leg. E.K.; 27 May 2002, net, 1 male, leg. E.K.; 6 July 2002, net, 1 male, 2 females, leg. E.K.; 25 July 2002, net, 1 male, leg. E.K.; 5 June 2004, net, 1 male, leg. E.K.; $23^{\circ} \mathrm{C}$, cloudy, rain, 29 July 2003 , net, 4 males, 1 female, leg. E.K.; $16^{\circ} \mathrm{C}$, windless, 20 September 2003, net, 3 males, leg. E.K. Władysławowo, beach, 27 July 2000, net, 1 female, leg. E.K. Southern Baltic Lakelands. Gdynia Bernadowo, Łęg nad Swelinią Reserve, 25 August 2011, light trap, 2 males, 5 females, leg. M.G.K.; 27 August 2011, light trap, 7 males, 2 females, leg. M.G.K.; 28 August 2011, light trap, 4 males, 1 female, leg. M.G.K.; 3 September 2011, light trap, 1 male, leg. M.G.K.; 5 September 2011, light trap, 1 male, leg. M.G.K.; 10 October 2011, light trap, 
2 males, leg. M.G.K.; 25 October 2011, light trap, 1 male, leg. M.G.K. Gdynia Kacze Buki, 21 July 2003, net, 3 males, leg. M.G.K. Szubin near Bydgoszcz, 26 July 1993, 1 male, leg. J.K. SUdETY MTS. Pokrzywna near Głuchołazy, 10 August 1983, at light, 1 female, leg. R.Sz.

Other specimens

Belgium: holotype D. bilineata, 1 đ̊: R.I.Sc.N.B. 18.073, coll et det., M. Goetghebuer, 354, Uccle, 1-9-17[1917], Lestage [leg. M. Lestage], det. Dasyhelea bilineata Goetgh., Dufouri, type $\widehat{\jmath}$, Goetghebuer, [IRSNB].

Distribution and biology

Norway, Finland, Russia (Karelia), Estonia, Great Britain, Ireland, Belgium, Germany, Poland, Czech Republic, Slovakia, France, Switzerland, Austria, Italy, Croatia, Hungary, Romania, Ukraine (Crimea), Bulgaria, Spain, Gibraltar, Algeria, Turkey (DomINIAK \& SZADZIEWSKI 2010).

Larvae of $D$. bilineata live in small and shallow water bodies like rock pools and their artificial equivalents (gutters, fountains, stoups, etc.), and reservoirs in leaf axils. Preimaginal stages are highly tolerant to desiccation and wide fluctuations in temperature (ZILAhi-SeBeSs 1931, VAlKanOV 1941, Disney 1975). Probably D. bilineata has the same survival strategy under adverse environmental conditions as observed in $D$. thompsoni DE MeILlon and D. necrophila SPINELli \& RoDRIGEZ. In the latter species, the development of first instar larvae, exposed to desiccation, takes place inside the egg shell (RONDEROS et al. 2006), while the larvae of $D$. thompsoni encyst in mucous capsules (CANTRELl \& MCLACHLAN 1982, MCLACHLAN \& LADLE 2001).

This species is known from numerous localities in Poland, especially in the northern part of the county. Adults fly from May to October. The larvae can be found all the year round (ASHE et al. 2007).

\section{Discussion}

Dasyhelea bilineata is closely allied to D. flavifrons (see below). SzADZIEWSKI $\&$ DOMINIAK (2006) mentioned that the males of these two species differ in the shape of the gonostylus and third palpal segment (expressed as the palpal ratio). Currently, only the latter character is considered suitable for the species identification.

In the year 1911 KIEFFER described D. halophila from Croatia, which seems to be very similar if not identical to $D$. bilineata. Larvae of this species were found in rockpools on the seashore. Unfortunately, the type specimens of $D$. halophila have most probably been lost or destroyed. Despite efforts to collect larvae from the locus typicus, no new materials have been obtained. D. halophila is currently treated as nomen dubium (DomINIAK \& SZADZIEWSKI 2010).

\section{Dasyhelea flavifrons (GUÉRIN, 1833)}

(Figs 32, 33, 34, 35, 36, 117, 118, 120, 121, 122, 123, 125)

Ceratopogon flavifrons GUÉRIN, 1833: 165 (description, figure - pupa, male, female, breeding site - sap of elm, France). 
Dasyhelea flavifrons: KIEFFER 1919: 51 (in key); KIEFFER 1925c: 62 (description - male, female); THIENEMANN 1925: 105, 108 (in key - larva, pupa); EDWARDS 1926: 403 (taxonomical comments); SZADZIEWSKI \& DOMINIAK 2006: 140 (description and figure - male, female, syn.: = obscurus, $=$ versicolor,$=$ dufouri, = hippocastani, = brevitibialis, = goetghebueri, = lignicola, = sensualis, = paludicola, $=$ oppress, , = septuosa, breeding site - sap and tree holes of various tree species, Poland, USA); DominiaK \& SZADZIEWSKi 2006: 5 (Poland); DominiaK et al. 2007b: 4 (Poland); DominiaK \& SzADZIEwSKi 2010: 13 (Bulgaria, Spain, Switzerland, Ukraine); GwiZDALSKA-KENTZER 2011: 37 (Poland).

Ceratopogon obscurus WINNERTZ, 1852: 45 (description and figure - female, Germany).

Dasyhelea obscura: KeILIN 1921: 576 (description and figure - egg, larva, pupa, breeding site - sap of elm and horse chestnut, biology, Great Britain, from Edwards, personal communication: decaying roots of Angelica, sap of oak, elm, hornbeam, debris of chestnut tree, stagnant water in tree-hole of oak); KIEFFER 1925c: 69 (description - female); THIENEMANN 1925: 105, 108 (in key - larva, pupa); KEILIN 1927: 369 (breeding site - sap of horse chestnut, biology, Great Britain); GoETGHEBUER 1934: 34 (comparison with versicolor, breeding site - from EDWARDS: decaying roots of Angelica).

Ceratopogon versicolor WINNERTZ, 1852: 45 (description and figure - female, Germany).

Culicoides versicolor: GOETGHEBUER 1914: 181 (description and figure - larva, pupa).

Dasyhelea versicolor: KIEFFER 1919: 50 (in key); GOETGHEBUER 1920: 46 (description and figure, in key - male, female, breeding site - sap of poplar, Belgium); KeILIN 1921: 585 (breeding site humus surrounding roots of meadowsweet, Great Britain); EDWARDS 1926: 403 (description, in key, breeding site - sap of elm and other trees, roots of greater burdock, Great Britain); GoETGHeBUer 1934: 36 (description and figure, in key - male, female, syn.: = goetghebueri, $=$ brevitibialis, $=$ ? flavifrons, = hippocastani); KACZOROWSKA 1996: 49 (description and figure - larva, pupa, male, female, breeding site - sap of horse chestnut and silver birch, syn.: = hippocastani, $=$ brevitibialis, = goetghebueri, Poland); FÜRST VON LIEVEN 1998: 51 (larval mouthparts, breeding site - sap of oak, horse chestnut, poplar, elm, Germany); DOMINIAK 2005: 5 (breeding site - sap and tree holes, Poland).

Ceratopogon dufouri LABOUlBÈNE, 1869: 158 (description and figure - larva, pupa, male, female, breeding site - sap of elm, France).

Dasyhelea dufouri: KIEFFER 1919: 51 (in key); KIEFFER, 1925c: 62 (description - male, female); STRENZKE 1950: 186 (syn.: = sensualis).

Ceratopogon hippocastani MIK, 1888: 185 (description and figure - larva, pupa, male, female, breeding site - sap of horse chestnut, Austria).

Dasyhelea hippocastani: KIEFFER 1919: 50 (in key).

Dasyhelea brevitibialis GoETGHEBUER, 1919: 72 (unnecessary new name for Culicoides versicolor, larva, pupa, breeding site - sap of poplar, Belgium); KIEFFER 1925c: 67 (description - male, female); THIENEMANN 1925: 105, 108 (in key - larva, pupa).

Dasyhelea goetghebueri KIEFFER, 1919: 53 (in key - male, Belgium); KIEFFER 1925c: 64 (description - male).

Dasyhelea lignicola KIEFFER, 1919: 57 (description and figure, in key - male, female, breeding site - tree holes, Czech Republic); Thienemann 1925: 105, 108 (in key - larva, pupa); Goetghebuer 1934: 33 (description - male, female, figure - female).

Dasyhelea sensualis KIEFFER, 1919: 55 (description, in key - male, female, figure - female, Istrian 
Peninsula, Crete); ThiENEMAnN 1925: 105, 108 (in key - larva, pupa); THIENEMANN 1950: 167 (syn.: = dufouri, breeding site - tree holes, Austria).

Dasyhelea paludicola KIEFFER, 1925a: 152 (description - female, Estonia); GOETGHEBUER 1934: 35 (description - female); REMM 1962: 114 (description - male, female, figure - male, Estonia); LUKASHEVA 1987: 28 (breeding site - sap of tree, Russia: Karachay-Cherkessia).

Dasyhelea geleiana: MAYER 1937: 301 (breeding site - sap of elm, Germany). Nec D. geleiana ZILAHI-SEBess, 1940.

Dasyhelea oppressa THOMSEN, 1935: 285 (description and figure - female, USA); WAUGH \& WIRTH 1976: 230 (description and figure - male, female, breeding site - sap of elm and oak, tree holes, USA); GRAVES \& GRAVES 1985: 88 (breeding site - shelf fungi, USA: North Carolina); WiLKENING et al. 1985: 519 (USA: Florida); HRIBAR \& GROGAN 2005: 231 (USA: Florida Keys).

Dasyhelea septuosa BORKENT in BORKENT \& WIRTH 1997: 58 (new name for D. obscura (WINNERTZ, 1852)).

\section{Diagnosis}

Wing length of male 1.1-1.5 mm, of female 1.1-1.4 mm. Third palpal segment short. Male palpal ratio $\mathrm{PR}_{(\mathrm{III})}$ less than 4.0. Subgenital plate evenly sclerotized, with a pair of small lateral projections and notum in the shape of an equilateral triangle.
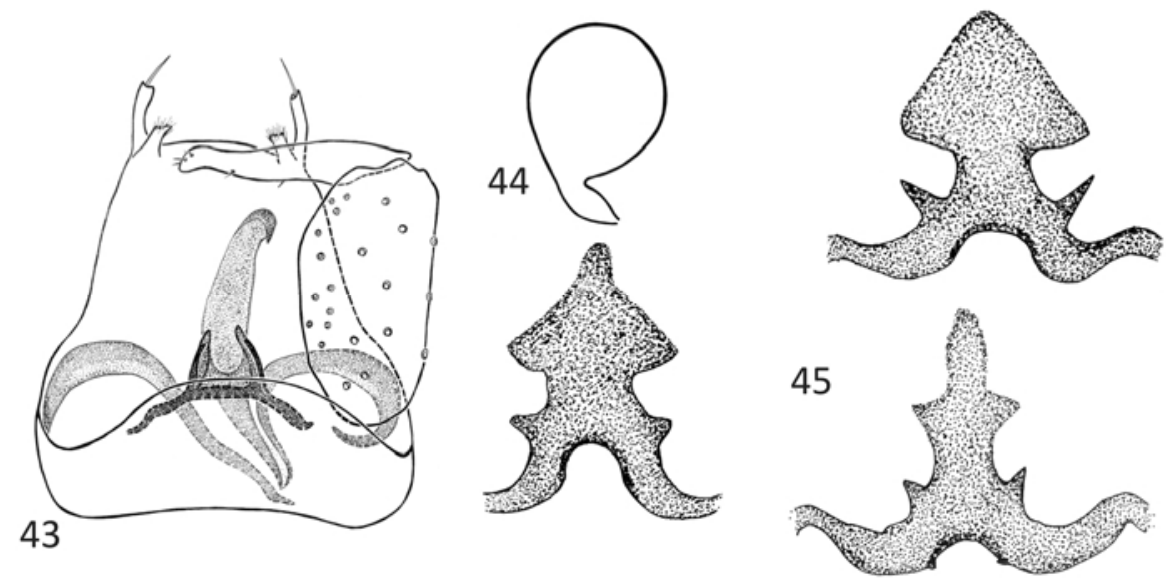

Figs 43-45. Dasyhelea flavifrons (GUÉRIN): 43 - male genitalia, 44 - spermatheca, 45 - female subgenital plate.

\section{Description}

Male. AR 0.89-0.98 $(\mathrm{n}=11)$. Palpal segment 3 short, cylindrical (Fig. 32), 58-70 $\mu \mathrm{m}$ long $(\mathrm{n}=11), \mathrm{PR}_{(\mathrm{III})} 2.42-3.89(\mathrm{n}=11)$. Scutellum dark or yellow, with 8-13 bristles and a few setae (Fig. 121). Wing length 1.14-1.54 mm $(\mathrm{n}=11)$, CR 0.49-0.55 ( $\mathrm{n}=11)$. Legs unicoloured or with some darker bands. Genitalia (Fig. 34). Posterior margin of sternite 
9 low-arched. Small, mesoventral hook on gonocoxite present. Gonostylus slender, usually slightly wavy. Parameres asymmetrical, posterior process very often markedly fused to right basal arm. Aedeagus as in D. bilineata.

Female. AR 0.71-0.86 $(\mathrm{n}=12)$. Palpal segment 3 short and stout (Fig. 33), 49-78 $\mu \mathrm{m}$ long $(\mathrm{n}=12), \mathrm{PR}_{\text {(III) }} 2.33-3.25(\mathrm{n}=12)$. Scutellum dark or yellow, bearing 10-11 bristles and some additional setae. Wing length 1.11-1.41 mm $(\mathrm{n}=12)$, CR 0.51-0.55 $(\mathrm{n}=12)$. Coloration of legs similar as in male. Genitalia (Figs 44, 45). Subgenital plate usually evenly sclerotized, with a pair of small and sharp pointed lateral processes and notum in the shape of an equilateral triangle. Spermatheca retort-shaped, length 78-100 $\mu \mathrm{m}(\mathrm{n}=10)$, width 51-64 $\mu \mathrm{m}(\mathrm{n}=11)$.

Material examined $(n=3112)$

Poland: Southern BaLTiC COASTLAndS. Elbląg, Aesculus hippocastanum L., sap, 30 May 2004, ex cult., 10-18 July 2004, 2 females, leg. P.D.; ex cult., 31 July -4 August 2004, 1 female, leg. P.D. Gdańsk, tree hole, 26 April 1986, ex cult., 5 males, 7 females, leg. R.L. Gdańsk, Betula pendula Rotн, sap, 14 May 1989, 4 larvae, 42 pupae, 25 males, 15 females, leg. R.Sz. Gdańsk Oliwa, 11 June 1983, dead in water in tree hole, 1 female, leg. R.Sz.; tree hole, 27 June 1987, ex cult., 4 males, 10 females, leg. R.L.; Aesculus hippocastanum L., sap, 3 June 2004, ex cult., 4-7 June 2004, 36 males, 68 females, leg. P.D.; ex cult., 11-23 June 2004, 2 males, 4 females, leg. P.D.; ex cult., 2830 June 2004, 1 pupa, 128 males, 144 females, leg. P.D.; ex cult., 30 June - 1 July 2004, 43 males, 72 females, leg. P.D.; ex cult., 7-8 July 2004, 17 males, 20 females, leg. P.D.; ex cult., 10-18 July 2004, 3 males, 2 females, leg. P.D.; ex cult., 19-25 July 2004, 1 exuvia, 2 males, 1 female, leg. P.D.; ex cult., 8-10 August 2004, 1 male, 1 female, leg. P.D.; ex cult., 21-23 August 2004, 1 female, leg. P.D.; ex cult., 24 October 2004, 1 larva, 1 pupa, leg. P.D. Gdańsk Politechnika, Aesculus hippocastanum L., sap, 1 June 2004, ex cult., 8 June 2004, 2 males, leg. P.D.; ex cult., 9-10 June 2004, 5 males, 2 females, leg. P.D.; ex cult., 11-23 June 2004, 12 males, 11 females, leg. P.D.; ex cult., 29-30 June 2004, 1 female, leg. P.D.; 3 July 2004, ex cult., 4-7 July 2004, 1 female, leg. P.D.; ex cult., 10-18 July 2004, 8 males, 8 females, leg. P.D.; ex cult., 19-25 July 2004, 16 males, 16 females, leg. P.D.; ex cult., 19-27 July 2004, 13 males, 14 females, leg. P.D.; ex cult., 27-28 July 2004, 5 males, 6 females, leg. P.D.; ex cult., 30-31 July 2004, 15 males, 15 females, leg. P.D.; ex cult., 31 July -4 August 2004, 8 males, 21 females, leg. P.D.; ex cult., 5-7 August 2004, 2 pupae, 12 males, 7 females, leg. P.D.; ex cult., 8-10 August 2004, 1 pupa, 10 males, 11 females, leg. P.D.; ex cult., 11-13 August 2004, 4 males, 5 females, leg. P.D.; ex cult., 14-16 August 2004, 4 males, 4 females, leg. P.D.; ex cult., 17-20 August 2004, 3 females, leg. P.D.; ex cult., 21-23 August 2004, 1 male, leg. P.D.; ex cult., 24-25 August 2004, 1 male, 1 female, leg. P.D.; ex cult., 2-4 September 2004, 1 female, leg. P.D.; ex cult., 4-6 September 2004, 1 male, leg. P.D.; 9 August 2004, ex cult., 14-16 August 2004, 28 males, 24 females, leg. P.D.; ex cult., 2-4 September 2004, 1 pupa, 23 males, 29 females, leg. P.D.; ex cult., 4-6 September 2004, 22 males, 35 females, leg. P.D.; ex cult., 7-10 September 2004, 1 female, leg. P.D.; ex cult., 22-28 September 2004, 1 pupa, 1 male, 8 females, leg. P.D.; 23 June 2006, ex cult., 1 larva, 58 males, 26 females, leg. P.D.; 23 January 2009, ex cult., 10-13 February 2009, 22 males, 23 females, leg. P.D.; tree hole, 7 July 2004, ex cult., 7 July 2004, 1 female, leg. P.D.; ex cult., 5-7 August 2004, 1 male, leg. P.D.; ex cult., 8 July 2004, 2 males, leg. P.D.; ex cult., 27-28 July 2004, 1 male, 1 female, leg. P.D.; ex cult., 31 July - 4 August 2004, 1 female, leg. P.D.; ex cult., 14-16 August 2004, 1 female, leg. P.D.; 5 April 2005, ex cult., 9 May 
2005, 3 males, 6 females, leg. P.D.; ex cult., 30 May - 1 June 2005, 1 female, leg. P.D.; Tilia platyphyllos SCOP., sap, 1 June 2004, ex cult., 1 June 2004, 1 male, leg. P.D.; ex cult., 2 June 2004, 7 males, 5 females, leg. P.D.; ex cult., 2 June 2004, 2 pupae, 3 June 20042 males, leg. P.D.; ex cult., 2 June 2004 pupa, 4 June 20041 male, leg. P.D.; ex cult., 2-4 June 2004, 4 pupae, 38 males, 50 females, leg. P.D.; ex cult., 4-7 June 2004, 7 pupae, 65 males, 67 females, leg. P.D.; ex cult., 6 June 2004, 1 male, leg. P.D.; ex cult., 9-10 June 2004, 1 pupa, 24 males, 27 females, leg. P.D.; ex cult., 11-23 June 2004, 12 males, 37 females, leg. P.D.; ex cult., 25-27 June 2004, 3 females, leg. P.D. Gdańsk Stocznia, Aesculus hippocastanum L., sap, 18 May 2004, ex cult., 1 June 2004, 3 males, 1 female, leg. P.D.; ex cult., 2 June 2004, 1 male, 1 female, leg. P.D.; ex cult., 3 June 2004, 2 males, 6 females, leg. P.D.; ex cult., 5-6 June 2004, 6 males, 12 females, leg. P.D.; ex cult., 7 June 2004, 2 males, 6 females, leg. P.D.; ex cult., 9-10 June 2004, 4 males, 8 females, leg. P.D.; 7 August 2004, ex cult., 17-30 October 2004, 2 pupae, 3 females, leg. P.D.; 9 August 2004, ex cult., 9-10 August 2004, 5 males, 15 females, leg. P.D.; ex cult., 17-20 August 2004, 21 males, 34 females, leg. P.D.; ex cult., 21-23 August 2004, 35 males, 33 females, leg. P.D.; ex cult., 24-25 August 2004, 8 males, 4 females, leg. P.D.; ex cult., 25-31 August 2004, 27 males, 27 females, leg. P.D.; ex cult., 7-10 September 2004, 1 pupa, 39 males, 34 females, leg. P.D.; ex cult., 11-13 September 2004, 2 pupae, 15 males, 38 females, leg. P.D.; ex cult., 13-19 September 2004, 3 males, 18 females, leg. P.D.; ex cult., 28 September - 10 October 2004, 12 pupae, 2 females, leg. P.D.; ex cult., 10-17 October 2004, 34 pupae, 1 male, 2 females, leg. P.D.; ex cult., 17-24 October 2004, 5 pupae, 2 males, 7 females, leg. P.D.; Tilia platyphyllos SCOP., sap, 9 August 2004, ex cult., 11-13 August 2004, 2 males, 1 female, leg. P.D.; ex cult., 14-16 August 2004, 3 males, leg. P.D.; ex cult., 17-20 August 2004, 3 males, 1 female, leg. P.D.; ex cult., 21-23 August 2004, 13 males, 6 females, leg. P.D.; ex cult., 24-25 August 2004, 5 males, 6 females, leg. P.D.; ex cult., 25-31 August 2004, 1 pupa, 14 males, 5 females, leg. P.D.; ex cult., 30 August - 1 September 2004, 3 pupae, 26 males, 54 females, leg. P.D.; ex cult., 2-4 September 2004, 3 pupae, 12 males, 13 females, leg. P.D.; ex cult., 4-6 September 2004, 5 pupae, 4 males, 13 females, leg. P.D.; ex cult., 7-10 September 2004, 7 males, 6 females, leg. P.D.; ex cult., 11-13 September 2004, 1 male, 6 females, leg. P.D.; ex cult., 13-19 September 2004, 1 pupa, 2 males, 7 females, leg. P.D.; ex cult., 19-22 September 2004, 2 pupae, 4 males, 12 females, leg. P.D.; ex cult., 18 September - 11 October 2004, 1 female, leg. P.D.; ex cult., 11-17 October 2004, 1 female, leg. P.D. Gdańsk Wrzeszcz, Aesculus hippocastanum L., sap, 17-25 May 1995, ex cult., 1 larva, 4 pupae, 1 male, leg. E.K.; 17-29 May 1995, ex cult., 3 males, 1 female, leg. E.K.; 17-25-29 May 1995, ex cult., 2 males, leg. E.K.; 21 May 1995, 1 larva, leg. E.K.; 21-24 May 1995, ex cult., 2 males, leg. E.K.; 21-26 May 1995, ex cult., 4 females, leg. E.K.; 22-24 May 1995, ex cult., 1 female, leg. E.K.; 24-30 May 1995, ex cult., 1 female, leg. E.K.; 17-26-29 June 1995, ex cult., 2 males, leg. E.K.; 22-26 June 1995, ex cult., 3 females, leg. E.K. Gdynia Wzgórze Św. Maksymiliana, Fagus sylvatica L., sap, 5 June 2004, ex cult., 10-23 June 2004, 2 males, 13 females, leg. P.D.; Populus nigra L., sap, 26 May 2004, ex cult., 26 May 2004, 1 female, leg. P.D.; ex cult., 27 May 2004, 1 pupa, 1 male, leg. P.D.; ex cult., 31 May 2004, 2 males, 1 female, leg. P.D.; ex cult., 4 June 2004, 1 male, leg. P.D. Kadyny near Elblag, Acer platanoides L., sap, 30 May 2004, ex cult., 8 June 2004, 1 male, leg. P.D.; ex cult., 28 June 2004, 1 female, leg. P.D.; ex cult., 1-2 July 2004, 1 male, 4 females, leg. P.D.; ex cult., 4-7 July 2004, 3 females, leg. P.D.; ex cult., 10-18 July 2004, 1 male, 1 female, leg. P.D.; Fagus sylvatica L., detritus in trunk cavity, 6 June 2004, ex cult., 4-7 July 2004, 3 males, 2 females, leg. P.D.; ex cult., 7-8 July 2004, 2 pupae, 1 female, leg. P.D.; ex cult., 10-18 July 2004, 1 female, leg. P.D.; sap, 6 June 2004, ex cult., 11-23 June 2004, 1 female, leg. P.D.; 
Populus balsamifera L., sap, 30 May 2004, ex cult., 31 May 2004, 5 females, leg. P.D.; ex cult., 2 June 2004, 1 male, 1 female, leg. P.D.; ex cult., 4 June 2004, 1 male, leg. P.D.; ex cult., 8 June 2004, 1 male, 2 females, leg. P.D.; ex cult., 9-10 June 2004, 10 males, 11 females, leg. P.D.; ex cult., 11-24 June 2004, 7 males, 11 females, leg. P.D.; Quercus petraea (MATT.) LieBL., sap, 6 June 2004, ex cult., 11-23 June 2004, 3 males, 1 female, leg. P.D. Malbork, Acer pseudoplatanus L., sap, 28 May 2004, ex cult., 11-30 June 2004, 10 males, 9 females, leg. P.D.; ex cult., 28 June 2004, 1 male, 3 females, leg. P.D.; ex cult., 30 June - 2 July 2004, 1 female, leg. P.D.; Aesculus hippocastanum L., sap, 25 May 2004, 1 larva, leg. P.D.; ex cult., 25-27 June 2004, 8 males, 5 females, leg. P.D.; ex cult., 29-30 June 2004, 2 males, 12 females, leg. P.D.; ex cult., 30 June - 2 July 2004, 1 male, 1 female, leg. P.D.; ex cult., 4-6 July 2004, 1 female, leg. P.D.; ex cult., 10-18 July 2004, 1 male, 1 female, leg. P.D.; Populus x canadensis MoEnch, sap, 10 July 2004, ex cult., 18 July 2004, 3 pupae, 2 males, 2 females, leg. P.D.; ex cult., 19-25 July 2004, 1 male, 3 females, leg. P.D.; ex cult., 8-10 August 2004, 1 male, leg. P.D.; ex cult., 21-23 August 2004, 1 female, leg. P.D.; ex cult., 30 August 1 September 2004, 1 female, leg. P.D.; ex cult., 7-10 September 2004, 1 male, leg. P.D. Mieroszyno near Władysławowo, Bielawskie Błota, 28 June 1979, 1 female, leg. R.Sz. Piaśnica near Wejherowo, tree hole, 29 June 1986, ex cult., 7 males, 2 females, leg. R.L. Sopot, 19 April 2005, ex cult., 25-28 May 2005, 1 male, leg. P.D. Strzebielino Morskie near Wejherowo, Alnus sp., tree hole, 8 May 1983, ex cult., 20 May 1983, 4 pupae, 1 male, 2 females, leg. R.Sz. Wejherowo, tree hole, 1 June 1986, ex cult., 1 female, leg. R.L.; 17 August 1986, ex cult., 2 pupae, 6 males, 10 females, leg. R.L.; near forester's house Migi, tree hole, 13 May 1986, ex cult., 4 males, 7 females, leg. R.L.; 6 June 1986, ex cult., 1 male, leg. R.L.; 9 June 1986, ex cult., 8 males, 3 females, leg. R.L.; on the Reda River, tree hole, 17 May 1986, ex cult., 1 male, 2 females, leg. R.L.; public garden, tree hole, 24 July 1987, ex cult., 2 males, 3 females, leg. R.L. Southern Baltic LAKELANDS. Gdańsk, Dolina Radości, Betula pendula Rотн, sap, 3-15 April 1989, ex cult., 1 larva, 5 males, 7 females, leg. R.Sz.; 3-20 April 1989, ex cult., 10 males, 14 females, leg. R.Sz.; 3-22 April 1989, 4 larvae, 60 pupae, 21 males, 23 females, leg. R.Sz.; 3-24 April 1989, ex cult., 2 larvae, 1 pupa, 13 males, 15 females, leg. R.Sz.; 4-13 April 1989, ex cult., 2 males, leg. R.Sz.; 4-15 April 1989, ex cult., 1 male, 1 female, leg. R.Sz.; 4-16 April 1989, ex cult., 1 pupa, 3 males, leg. R.Sz. Gdynia Bernadowo, Łęg nad Swelinią Reserve, 9 August 2011, net, 1 female, leg. M.G.K.; 25 August 2011, light trap, 1 male, leg. M.G.K.; 27 August 2011, light trap, 1 male, leg. M.G.K.; 28 August 2011, light trap, 1 male, leg. M.G.K.; 5 September 2011, light trap, 1 male, leg. M.G.K.; 10 October 2011, light trap, 1 male, leg. M.G.K. Głowaczewo near Wałcz, Betula pendula Roth, sap, 23 July 2004, ex cult., 31 July - 4 August 2004, 2 females, leg. P.D.; ex cult., 8-10 August 2004, 1 male, leg. P.D.; ex cult., 11-13 August 2004, 1 female, leg. P.D.; ex cult., 14-16 August 2004, 2 males, 5 females, leg. P.D.; ex cult., 17-20 August 2004, 17 males, 10 females, leg. P.D.; ex cult., 21-23 August 2004, 2 males, 4 females, leg. P.D.; ex cult., 24-25 August 2004, 2 males, 1 female, leg. P.D.; ex cult., 25-31 August 2004, 3 males, 2 females, leg. P.D.; ex cult., 30 August - 1 September 2004, 1 female, leg. P.D.; ex cult., 4-6 September 2004, 1 male, 1 female, leg. P.D.; ex cult., 7-10 September 2004, 5 females, leg. P.D.; ex cult., 11-13 September 2004, 1 male, 1 female, leg. P.D.; ex cult., 13-19 September 2004, 1 male, 1 female, leg. P.D.; ex cult., 22-28 September 2004, 1 male, leg. P.D. Krzeszna, 14 July 2003, net, 1 male, leg. M.G.K. Okole near Starogard Gdański, on the River Wierzyca, 15 June 2004, net, 3 males, leg. M.G.K. Sitnica near Wałcz, $53^{\circ} 06.251^{\prime} \mathrm{N} 15^{\circ} 54.752^{\prime} \mathrm{E}, 2 \mathrm{~m}$ BMSL, 18 June 2005, net, 2 males, leg. P.D. Załom near Wałcz, wet meadow near Lake Ostrowieckie, $53^{\circ} 05.693 \mathrm{~N} 16^{\circ} 00.385^{\prime} \mathrm{E}, 45 \mathrm{~m}$ AMSL, 19 June 2005, net, 1 male, leg. P.D. Szwecja near Wałcz, Acer platanoides L., sap, 22 July 2004, 
ex cult., 31 July - 4 August 2004, 2 males, leg. P.D.; ex cult., 8-10 August 2004, 1 pupa, 1 male, 4 females, leg. P.D.; ex cult., 11-13 August 2004, 1 female, leg. P.D.; ex cult., 14-16 August 2004, 1 male, leg. P.D.; ex cult., 17-21 August 2004, 1 female, leg. P.D.; ex cult., 21-23 August 2004, 1 female, leg. P.D. EAstern Baltic Lakelands. Mazurki near Augustów, peat bog forest, 15 May 2005, net, 1 male, leg. P.D. Wiatrołuża Pierwsza near Suwałki, Ulmus sp., sap, 3 July 2005, ex cult., 9 July 2005, 8 males, 3 females, leg. P.D.; ex cult., 16-17 July 2005, 7 males, 6 females, leg. P.D.; ex cult., 18-20 July 2005, 4 pupae, 12 males, 15 females, leg. P.D.; ex cult., 20-22 July 2005, 3 pupae, 6 males, 6 females, leg. P.D.; ex cult., 25 July -7 August 2005, 1 male, 4 females, leg. P.D. Silec near Kętrzyn, forest, May/2.1980, 5 males, leg. R.Sz. Żytkiejmy near Gołdap, 3 June 1981, 1 female, leg. R.Sz. Central Poland Lowlands. Przasnysz near Warszawa, 16 August 1993, net, 1 male, leg. K.G. PodLASIE. Białowieża, Carpinus betulus L., sap, 2 May 2004, 3 larvae, 2 pupae, leg. P.D.; ex cult., 16 May 2004, 1 female, leg. P.D.; ex cult., 27 May 2004, 6 males, 1 female, leg. P.D.; ex cult., 31 May 2004, 1 pupa, 5 males, 4 females, leg. P.D.; ex cult., 29-30 May 2004, 25 males, 3 females, leg. P.D.; ex cult., 1 June 2004, 4 males, 3 females, leg. P.D.; ex cult., 2 June 2004, 1 female, leg. P.D.; ex cult., 3 June 2004, 1 male, 1 female, leg. P.D.; ex cult., 4 June 2004, 1 male, leg. P.D.; ex cult., 5 June 2004, 1 pupa, 1 male, leg. P.D.; ex cult., 6 June 2004, 1 female, leg. P.D. Białowieża Forest, sector 668Cc, 17 June 1987, Moericke trap, 1 male, IZ PAN. SUDETY MTS. Karpacz, on the River Łomniczka, 11 July 2004, net, 1 female, leg. M.G.K. Pasterka near Kudowa Zdrój, stream near the Pośny Waterfall, 50²9.300'N 16²1.124'E, 641 m AMSL, 26 July 2006, net, 1 male, leg. P.D. Sosnówka Dolna near Jelenia Góra, 4 August 1982, net, 1 female, leg. R.Sz.; forest margin, 13 August 1982, net, 1 male, leg. R.Sz. Silesian-KraKów UPLAND. Ojców near Kraków, 23 June 1988, at light, 1 male, leg. Cz.M.; 24 June 1988, at light, 3 males, 2 females, leg. R.Sz. Central MaŁopolska Upland. Święta Katarzyna near Kielce, 5054.066'N 2053.563'E, 444 m, Abies alba Mill., sap, 29 July 2008, ex cult., 3 pupae, 21 males, 23 females, leg. P.D. Święta Katarzyna near Kielce, meadow on the River Czarna Woda, 50 55.158'N 2054.136'E, 276 m, 27 July 2008, net, 1 male, leg. P.D. Wola Szczygiełkowa near Kielce, near Różańcowe Łąki, 5054.591'N 2056.597'E, 245 m, 29 June 2008, net, 1 female, leg. P.D. Outer Western CARPATHians. Zawoja Składy, 2 June 2006, net, 1 male, leg. P.D. Central Western Carpathians. Czertezik in the Pieniny Mts., 22 June 1988, 6 males, 1 female, leg. R.Sz. Chyżne near Nowy Targ, Las Hamrzyska, 29 July 2006, net, 1 male, leg. P.D. Chyżne near Nowy Targ, Wyżni Młyn on the Jeleśnia Stream, 29 July 2006, net, 1 female, leg. P.D. Czarny Dunajec near Nowy Targ, Czarny Dunajec-Baligówka, 27 and 29 June 2006, Malaise trap, 1 female, leg. P.D. Czarny Dunajec near Nowy Targ, Baligówka peat bog, 26 June 2006, net, 4 males, 6 females, leg. P.D.; 28 June 2006, net, 10 males, 4 females, leg. P.D. Piekielnik near Nowy Targ, meadow near Puścizna Mała, 27 June 2006, net, 1 female, leg. P.D. Sokolica in the Pieniny Mts., 28 June 2003, net, 1 male, leg. M.G.K. Zakopane Huty, 6 August 1981, 1 female, leg. R.Sz. Zakopane Jaszczurówka, Potok Olczyski, 8 August 1981, 2 females, leg. R.Sz. EASTERN BESKID MTS. Muczne near Ustrzyki Górne, Tarnawa Reserve, peat bog, 13 July 2005, net, 1 male, leg. P.D. Wołosate near Ustrzyki Górne, Wołosate Reserve, peat bog, 12 July 2005, net, 1 female, leg. P.D. Ustrzyki Górne, 23-30 July 1980, 9 females, leg. R.Sz.; on the River Wołosaty, 28 July 1980, 2 males, leg. R.Sz.; Apiaceae, 12 July 2005, net, 1 male, leg. P.D.

\section{Distribution and biology}

Estonia, Great Britain, Belgium, Germany, Poland, Czech Republic, France, Austria, Switzerland, Spain, Croatia (Istria), Greece (Crete), Bulgaria, Ukraine (Crimea), Russia (Karachay-Cherkessia), USA (DoMINIAK \& SZADZIEWSKI 2010). 
Most of the specimens used in this study were reared from larvae collected from sap oozing from trees or from tree holes. The preimaginal stages of $D$. flavifrons can be found in Poland all the year round; the adult flight period is from May to October. This species is known from numerous localities in Poland.

Discussion

Dasyhelea flavifrons has been described many times under different names, but the type specimens of most of them have been destroyed or lost (SZADZIEWSKI \& DOMINIAK 2006). The interpretation of two species described by Winnertz, namely D. obscura and D. versicolor, follows Goetghebuer $(1914,1920)$ and KeILIN $(1921)$, who used these names for Dasyhelea midges reared from tree sap.

\section{Dasyhelea caesia REMM, 1993}

(Figs 21, 27, 46)

Dasyhelea caesia Remm, 1993: 193 (description and figure - male, female, Russia: Yakutia).

Dasyhelea lugensis BRODSKAYA, 1995: 9 (description and figure - larva, pupa, male, female, breeding site - small water body, Russia: Leningrad Oblast); PRZHIBORO 2004: 105 (breeding site - lake margin, Russia: Leningrad Oblast); PRZHIBORO 2005: 166 (breeding site - lake margin with Sphagnum and Carex, Russia: Leningrad Oblast); DominiaK \& SzADZIEwski 2008: 149 (Poland); DOMINIAK \& SZADZIEWSKI 2010: 18 (distribution). Syn. n.

\section{Diagnosis}

Posterior process straight, nearly perpendicular to the basal arms, narrow; apical part wedge-shaped, with fine hair. Submedian projections of aedeagus long and thin, wavy. Posterior margin of sternite 9 extended, covered with short preapical hair.

\section{Description}

Male. AR 0.87-0.91 $(\mathrm{n}=4)$. Clypeus bearing 5-9 setae (Fig. 27). Palpal segment 3 short, cylindrical; length 43-53 $\mu \mathrm{m}(\mathrm{n}=6), \mathrm{PR}_{\text {(III) }} 2.05-3.12(\mathrm{n}=6)$. Transverse suture poorly defined. Scutellum paler than scutum, usually tawny, with 7-9 bristles. Wing with one radial cell (Fig. 21); length 1.08-1.21 mm $(\mathrm{n}=6)$, CR 0.45-0.47 $(\mathrm{n}=6)$. Legs paler than thorax, unicoloured, with black knees. Genitalia (Fig. 46). Posterior margin of sternite 9 slightly extended and covered with fine setae. Gonocoxite slender, lacking mesoventral hook. Gonostylus long, with slightly bent and pointed apex. Parameres asymmetrical. Basal arms ribbon-like. Right basal arm and posterior process fused together by hyaline membrane. Posterior process straight, wedge-shaped, covered with short hair in apical part. Aedeagus broad, with two long, thin and wavy submedian projections. Sometimes, a second pair of shorter and much more slender processes is also well visible.

Female. Subgenital plate arch-shaped, sometimes with rounded or irregular, weakly sclerotized notum. Spermatheca single, retort-shaped (REMM 1993, BRODSKAYA 1995). 


\section{Material examined $(n=10)$}

Type material. Russia: holotype male and three paratypes (female and two male genitalia) pinned together, labelled as follows: Yakutia, Tektyur, H. Remm, 21.VI.68, holotypus, paratypus, Dasyhelea caesia Remm, coll. Remm, Das. caesia Holotypus. [TUZ].

Poland: Southern Baltic Coastlands. Krzewsk near Elblagg, 10 July 1983, 1 male, leg. R.Sz. Eastern Baltic Lakelands. Michnowce near Sejny, 15 August 2007, net, 2 males, leg. P.D. Central Poland Lowlands. Czeszów near Wrocław, gravel pit, 30 August 2007, net, 2 males, leg. P.D. EASTERN MAŁOPOLSKA UPLAND. Zwierzyniec near Zamość, backwater of River Wieprz, 5 July 2006, net, 1 male, leg. P.D.

\section{Distribution and biology}

Russia (Leningrad Oblast, Yakutia), Poland (DOMINIAK \& SzADZIEWSKI 2010).

In Russia the preimaginal stages (last instar larvae and pupae) of Dasyhelea caesia were collected in July, from a small water body and the littoral zone of lakes (BRODSKAYA 1995, PRZHIBORO 2005).

So far, this species has been reported from four localities in Poland.

\section{Discussion}

An examination of the types of $D$. caesia showed that the species is identical with D. lugensis BRODSKAYA, a species described from the Leningrad Oblast in Russia. There are no differences in the shape of the male genitalia and female subgenital plate between these two species. I therefore consider these two names conspecific and propose to treat $D$. lugensis as a new junior synonym of $D$. caesia. New synonymy.

46
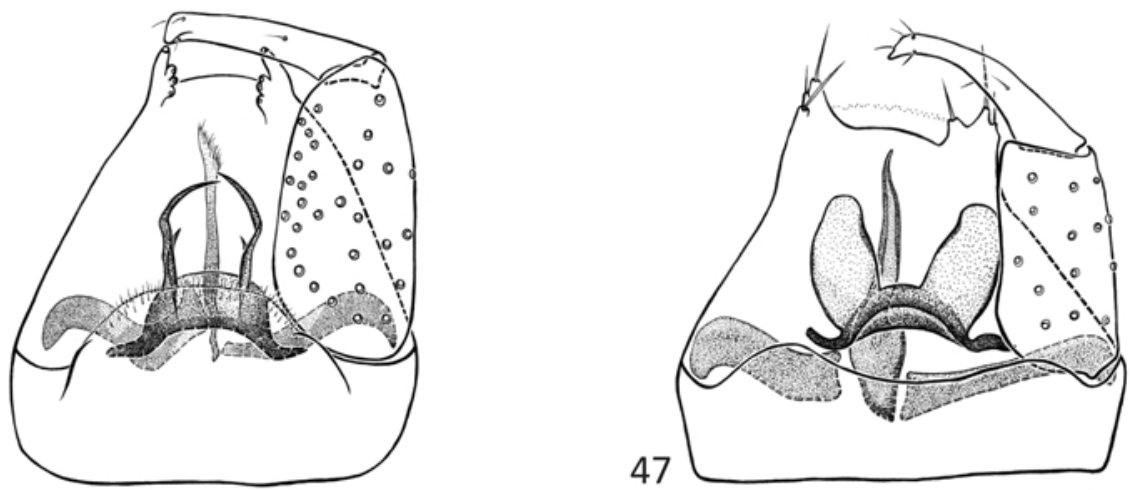

Figs 46-47. Male genitalia: 46 - Dasyhelea caesia Remm; 47 - D. stackelbergi Remm.

\section{Dasyhelea stackelbergi REMM, 1993}

(Fig. 47)

Dasyhelea stackelbergi ReMm, 1993: 194 (description and figure - male, Russia: Leningrad Oblast); DOMINIAK \& SZADZIEWSKI 2006: 5 (Poland); DOMINIAK \& SZADZIEWSKI 2010: 25 (distribution). 


\section{Diagnosis}

Posterior process straight, nearly perpendicular to the basal arms, very stout basally and tapered to a point. Submedian projections of aedeagus lobate. Posterior margin of sternite 9 slightly concave.

\section{Description}

Male. AR $0.89(\mathrm{n}=1)$. Third palpal segment cylindrical, $55 \mu \mathrm{m}$ long $(\mathrm{n}=1), \mathrm{PR}_{(\mathrm{III})} 2.50$ $(\mathrm{n}=1)$. Transverse suture absent. Scutellum dark. Wing length $1.07 \mathrm{~mm}(\mathrm{n}=1), \mathrm{CR} 0.44$ $(\mathrm{n}=1)$. Legs dark, tibiae somewhat paler than femora, knees black. Genitalia (Fig. 47). Apicolateral processes of tergite 9 conical, short and broad. Posterior margin of sternite 9 with shallow excavation. Gonocoxite without mesoventral hook. Gonostylus slightly curved, with sharply pointed apex. Parameres asymmetrical, divided. Posterior process straight, nearly perpendicular to the basal arms, very stout basally but tapering rapidly to a point. Basal arch of aedeagus rather low and narrow. Submedian projections of aedeagus lobate, membranous, well sclerotized only along the margins.

Female. Unknown.

Material examined $(\mathbf{n}=\mathbf{1})$

Poland: EASTERn Baltic LaKelands. Słupie near Suwałki, near the River Czarna Hańcza, peat bog, 5 July 2005 , net, 1 male, leg. P.D.

\section{Distribution and biology}

Russia (Leningrad Oblast), Poland (SZADZIEWSKI \& DOMINIAK 2010).

The record of $D$. stackelbergi is based on one male collected on a peat bog in north-east Poland. In Russia adults fly from June to August (REMM 1993).

\section{Discussion}

REMM (1993) included both sexes in the type series (paratypes: 1 male, 4 females), but he neither described nor illustrated a female of this species. D. stackelbergi is very similar to $D$. unicolor REMM, but differs in the shape of the posterior process of the parameres and the submedian processes of the aedeagus.

Type specimens of $D$. stackelbergi are deposited in the collection of the Zoological Institute of the Russian Academy of Sciences in St. Petersburg (REMM 1993).

\section{subgenus Dicryptoscena}

Dicryptoscena ENDERLEIN, 1936: 51. type species Dasyhelea inclusa KIEFFER, 1918: 188 (by original designation).

\section{Diagnosis}

Frontal sclerite elongate, club-shaped or slightly rhomboid, with lateral lobes and sometimes with additional apicolateral borders. Last flagellomere with conical 


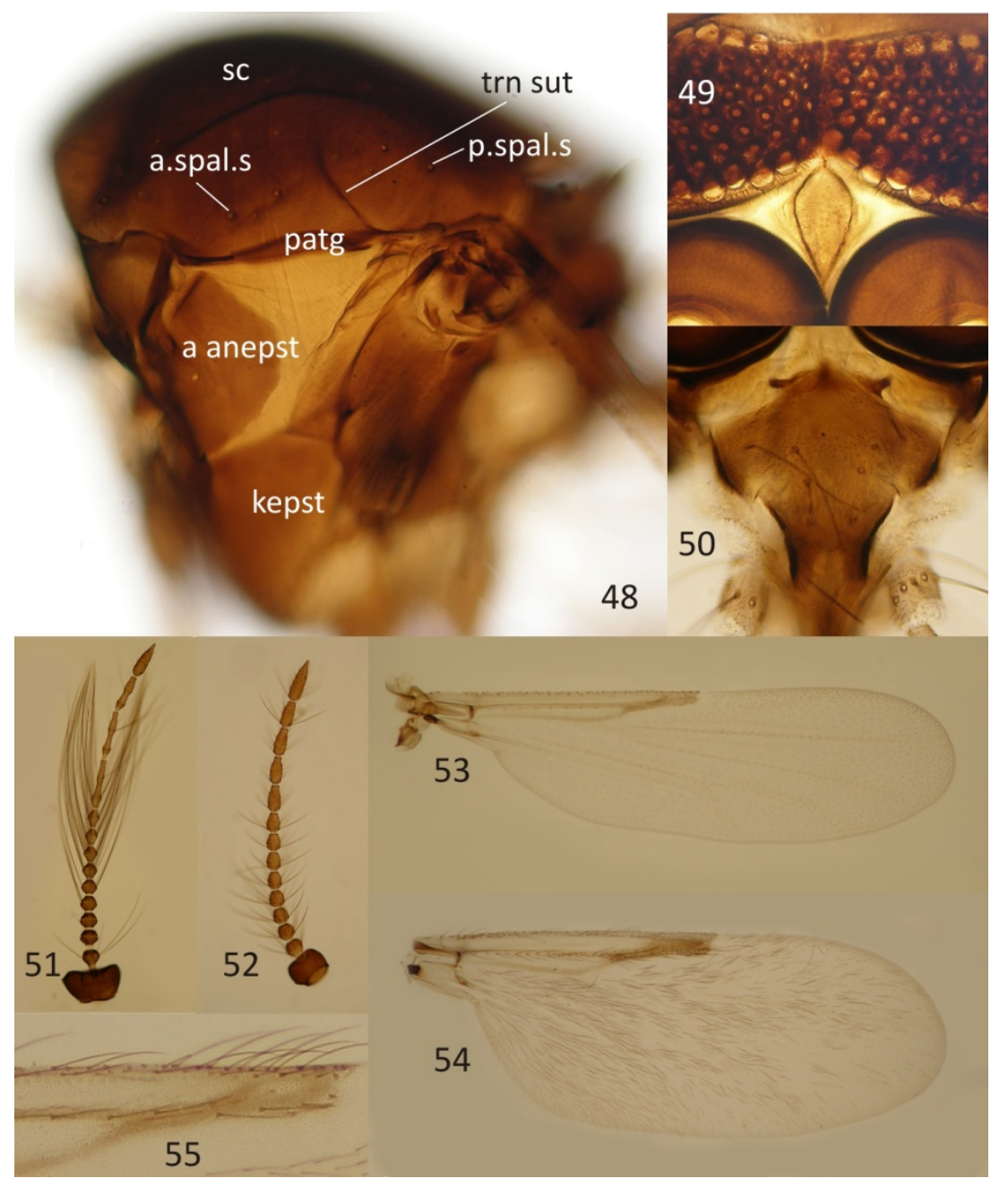

Figs 48-55. Dasyhelea lucida Remm, male (55), D. modesta (WinnerTz), female (52, 54), D. stellata Remm, male $(49,50)$, D. thienemanni Spătaru \& DAMiAn-GeorgESCU, male $(48,51,53)$.

48 - thorax, 49 - frontal sclerite, 50 - clypeus, 51, 52 - antenna, 53, 54 - wing, 55 - radial cells.

Abbreviations: a anepst - anteroanepisternite, patg - paratergite, sc - scutum, a.spal.s - anterior supraalar setae, p.spal.s - posterior supraalar setae, trn sut - transverse suture.

prolongation. Clypeus short, in the form of single sclerite with lobes, nearly pentagonal. Third palpal segment slender, as long as or longer than the next two combined. Transverse suture well defined. Wing with two radial cells, with first cell $r_{1}$ narrow and slit-like. 
Male. Apicolateral processes of tergite 9 short. Posterior margin of sternite 9 extended, bilobed, with horn-like submedian processes, fused to aedeagus. In some species apex of sternite 9 markedly bent inwards. Gonostylus long, slightly curved. Parameres asymmetrical. Posterior process usually long, fused to right basal arm.

Female. Antennal segments without reticulation, distal flagellomeres elongate. Subgenital plate arch-shaped, with club-shaped, conical or irregular notum. The latter very often with weakly sclerotized base. Spermatheca single, subspherical or ovoid, with short neck or without neck.

\section{Discussion}

Some species of the traverae group (WIRTH 1952) and the leptobranchia group (WAUGH \& WIRTH 1976) belong to the subgenus Dicryptoscena. The pupae of Dicryptoscena midges have very long and annulated respiratory horns, with numerous small spiracles. The posterior processes of the anal segment are short, flattened and divergent.

The subgenus Dicryptoscena is represented by six species in the Polish fauna. The two other European species are D. fulvosa REMM and D. kurensis REMM (DOMINIAK \& SZADZIEWSKI 2010).

\section{Key to species}

1. Fifth palpal segment 1.5 times longer than fourth palpal segment, with two rows of long setae (Fig. 9) modesta

-. Fifth palpal segment as long as fourth palpal segment, with single row of long setae ......2

2. Gonostylus with slender apex (Fig. 56, 59, 60, 61) . .3

-. Gonostylus with bulbous apex (Fig. 62, 63

3. Posterior margin of male sternite 9 with two short and divergent horn-like projections. Posterior process distinctly stout mesally (Fig. 59) notata

-. Posterior margin of male sternite 9 bent inwards. Posterior process tapering to a point (Fig. 60, 61) .. .4

4. Posterior process stout, sometimes with slightly recurved tip. Aedeagus with short, finger-like median projections (Fig. 60) lucida

-. Posterior process much more slender, markedly twisted. Aedeagus with long, strongly expanded distally submedian projections (Fig. 61)

5. Posterior margin of male sternite 9 with short projections. Submedian projections of aedeagus expanded distally (Fig. 62)

glukhovae

-. Posterior margin of male sternite 9 with very long projections. Submedian projections of aedeagus sharp (Fig. 63)

thienemanni 


\section{Dasyhelea modesta (WINNERTZ, 1852)}

(Figs 9, 11, 14, 52, 54, 56, 57, 58, 119)

Ceratopogon modestus WinNERTZ, 1852: 43 (description and figure - female, Germany).

Dasyhelea modesta: KIEFFER 1919: 50 (in key); KIEFFER 1925c: 65 (description, in key - male, female, figure - male); GOETGHEBUER 1927: 95 (figure - female, Belgium); GOETGHEBUER 1932: 125 (description - male); GOETGHEBUER 1934: 34 (description, in key - male, female, figure female); MAYER 1934a: 225 (description - larva, pupa, figure - pupa, breeding site - salt water, Germany); Goetghebuer \& Timon David 1937: 411 (France); Zilahi-Sebess 1940: 51 (description, in key - male, female, figure - male, syn.: = strobli, Hungary); DE MEIJERE 1946: 8 (the Netherlands); THIENEMANN 1950: 166 (breeding site - lakes, ponds, mud, swamps, Austria); THIENEMANN 1954: 614 (syn.: = longipalpis, = halophila); REMM 1962: 110 (description - male, female, figure - male, syn.: = strobli, Estonia); ReMm 1966: 58 (Lithuania); ReMm 1967: 13 (Georgia, Russia: North Ossetia); Remm 1969: 208 (in key, figure - male); Remm 1973b: 354 (Hungary); HAVELKA 1976a: 75 (Germany); HAVELKA 1976b: 226 (description and figure female); ReMm 1979: 49 (Estonia); ReMm 1981: 29 (syn.: = aestivus, = densipilosa); SZADZIEWSKI 1983: 66 (Poland); SZADZIEWSKI 1986: 38 (description and figure - male, syn.: $=$ aestivus, $=$ longipalpis, $=$ strobli,$=$ pratensis, $=$ bihamata,$=$ moascari,$=$ ?densipilosa, breeding site - saline soil, Algeria, Belgium, Egypt, Iran, Poland, neotype for D. bihamata KIEFFER, 1923); DeléCOlle \& Rieb 1990: 184 (France); Gosseries 1991: 42 (syn.: = aestiva, = pratensis); DelÉCOLle \& Rieb 1993: 111 (Spain); KNOZ 1996: 87 (the Netherlands); KNOZ 1997: 81 (Czech Republic); KNOZ 1998: 118 (Czech Republic); CHANDLER 1998: 55 (= holosericea sensu EDWARDS 1926, syn.: = aestiva, Great Britain); SZADZIEWSKI 1998: 103 (Switzerland); PRZHiBORO 1999: 130 (breeding site - lake margin, Russia: Karelia); HAgAN et al. 2000: 471 (Norway); DelÉCOLLE 2002: 28 (syn.: = aestiva, = longipalpis, = strobli, = pratensis, = bihamata, $=$ moascari,$=$ densipilosa, Andorra); PRZHIBORO 2004: 105 (breeding site - lake margin, Russia: Leningrad Oblast); TótнovÁ et al. 2004a: 309 (Czech Republic); TótноvÁ et al. 2004b: 146 (Czech Republic); Yu et al. 2006: 324 (description and figure - male, China); ChandLer et al. 2008: 84 (figure - male, comments, breeding site - mud, Great Britain); DoMINIAK \& SZADZIEWSKI 2010: 22 (syn.: $=$ aestiva,$=$ longipalpis, $=$ inclusa,$=$ strobli, $=$ pratensis, = bihamata, = holosericea sensu EDWARDS 1926, = moascari, = densipilosa, Afghanistan, Bulgaria, Spain); GwizDALSKA-KentZer 2011: 37 (Poland).

Ceratopogon aestivus WINNERTZ, 1852: 42 (description and figure - female, Germany).

Dasyhelea aestiva: KIEFFER 1919: 50 (in key); KIEFFER 1925c: 64 (description, in key - female); EDWARDS 1929: 425 (= holosericea sensu EDWARDS 1926); GOETGHEBUER 1934: 30 (description and figure, in key - female); GoetgheBUER \& TIMON DAVID 1937: 411 (France); Zilahi-SEbess 1940: 47 (description, in key - female, Hungary); DE MEIJERE 1946: 8 (the Netherlands); ReMm 1962: 110 (description - male, female, figure - male, syn.: = ?pratensis, Estonia); ReMm 1967: 14 (Azerbaijan, Georgia); ReMm \& ZHoGolev 1968: 831 (Ukraine: Crimea); DAMIAN-GEORGESCU 1973: 453 (description - male, figure - male, female, Romania); REMM 1973b: 354 (Hungary); HAVELKa \& AGUILAR 1999: 36 (syn.: = modestus, = longipalpis, $=$ densipilosa,$=$ ? bihamata) .

Dasyhelea longipalpis KIEFFER, 1913c: 37 (description - male, Germany); KIEFFER 1915: 65 (description - male); RIETH 1915: 424, 425 (description and figure - pupa, breeding site - salt water, peat bog, Germany, Sweden); THIENEMANN 1915: 446 (breeding site - saline habitats, peat bog, comments, Germany, Sweden); Thienemann 1925: 103 (breeding site - salt water, 
comments, Germany); GoETGHEBUER 1934: 33 (description and figure, in key - male).

Dasyhelea inclusa KIEFFER, 1918: 188 (description, in key - male, Czech Republic); GoETGHEBUER 1934: 33 (description, in key - male).

Dasyhelea strobli KIEFFER, 1919: 63 (new name for Ceratopogon versicolor var. obscurus STROBL nec WinNertz, description and figure, in key - female, Spain); Goetghebuer 1934: 36 (syn.: = versicolor var. obscurus STROBL nec WINNERTZ, description and figure, in key - female).

Dasyhelea pratensis GOETGHEBUER, 1920: 44 (description and figure, in key - male, Belgium); KIEFFER 1925c: 66 (description, in key - male); GOETGHEBUER 1934: 35 (description, in key - male).

Dasyhelea bihamata KIEFFER, 1923: 667 (description and figure, in key - male, Algeria); GOETGHEBUER 1934: 31 (description - male).

Dasyhelea holosericea: EDWARDS 1926: 402 (description - male, female, figure - female, syn.: $=$ ?aestiva, = pratensis, Great Britain). Nec D. holosericea (MEIGEN, 1804).

Dasyhelea tarsalis: KARL 1940: 30 (Poland). Nec D. tarsalis KIEFFER, 1919.

Dasyhelea moascari MACFIE, 1943: 153 (description, in key - male, female, figure - male, Egypt); BOORMAN \& VAN HARTEN 2002: 445 (description and figure - male, Yemen).

Dasyhelea densipilosa ToKUNAGA, 1963: 41 (description - male, female, Japan).

\section{Diagnosis}

The last palpal segment 1.5 times longer than fourth palpal segment, with two rows of long setae. Posterior margin of male sternite 9 with two horn-like projections separated by deep and moderately long furrow. Anterior part of female subgenital plate nearly triangular or spatula-like. Spermatheca large.

\section{Description}

Male. AR 1.04-1.17 $(\mathrm{n}=10)$. Clypeus entire, with 7-10 long setae (Fig. 11). Palpal segment 3 slender, 103-123 $\mu \mathrm{m}$ long $(\mathrm{n}=14), \mathrm{PR}_{\text {(III) }}$ 5.71-7.06 $(\mathrm{n}=14)$. Palpal segment 5 ca 1.5 times longer than palpal segment 4 , with additional row of long setae in the middle part (Fig. 9). Scutellum pale or dark, with 9-13 bristles and some smaller setae. Wing length 1.18-1.54 mm $(\mathrm{n}=13)$, CR 0.49-0.53 $(\mathrm{n}=13)$. Legs dark, especially knees, but tarsi paler. Genitalia (Fig. 56). Posterior margin of sternite 9 divided in the apical part into two hornlike processes, separated from each other by wedge-shaped, deep and moderately long furrow. Gonostylus with slender apex. Parameres asymmetrical. Posterior process tapering evenly to a point. Apicolateral processes of aedeagus conical, sometimes scrolled and then visible as a thin lamella.

Female. Antenna with distal flagellomeres elongate (Fig. 52), AR 0.89-1.05 $(\mathrm{n}=19)$. Palpal segment 3 with sensilla capitata (Fig. 119), length 83-122 $\mu \mathrm{m}(\mathrm{n}=20), \mathrm{PR}_{(\mathrm{III})} 4.30$ $5.60(\mathrm{n}=20)$. Palpal segment 5 as in male. Scutellum yellow, with 8-11 bristles and a few small setae. Wing with two radial cells, cell $r_{1}$ slit like, $r_{2}$ occluded (Fig. 54). Wing length 1.14-1.38 mm $(\mathrm{n}=19)$, CR 0.50-0.55 $(\mathrm{n}=19)$. Legs entirely dark, only tarsi paler. Genitalia (Figs 57, 58). Subgenital plate with notum weakly sclerotized at its base. Anterior part of notum nearly triangular or spatula-like. Spermatheca with short neck, ovoid, large, dimensions 87-146 $\mu \mathrm{m}(\mathrm{n}=11) \times 53-119 \mu \mathrm{m}(\mathrm{n}=14)$. 


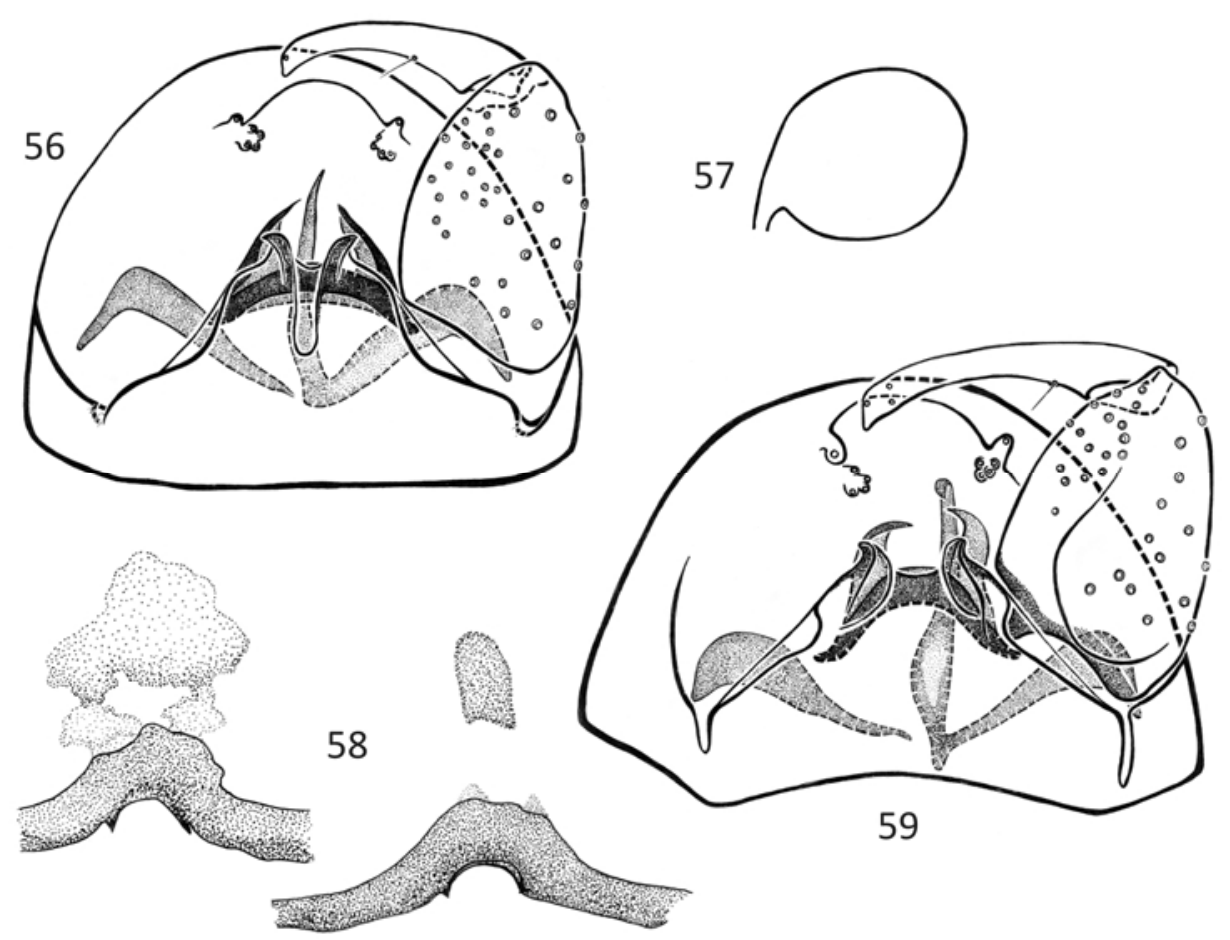

Figs 56-59. Dasyhelea modesta (WinNerTZ) $(56,57,58)$ and D. notata Goetghebuer (59): 56,59 - male genitalia, 57 - spermatheca, 58 - female subgenital plate.

Material examined $(n=43)$

Poland: Southern Baltic Coastlands. Gdańsk, in house, June 1977, 1 male, leg. R.Sz. Gdańsk, Sobieszewska Is., Górki Wschodnie, salt soil, 5 May 1975, ex cult., 2 males, 3 females, leg. R.Sz.; 25 May 1977, ex cult., 1 male, 1 female, leg. R.Sz.; on the River Wisła Śmiała, salt mud, 5-18 May 1977, ex cult., 1 male, leg. R.Sz.; beach, 29 July 2004, net, 1 male, leg. E.K.; 12 July 2005, net, 1 male, 1 female, leg. E.K. Mechelinki near Gdynia, saline habitats, 20 August 2006, 1 female, leg. K.B. Sopot, beach, 20 September 2003, net, 1 male, leg. E.K. Wierzchucino near Żarnowiec, 25 April 1984, 2 males, 2 females, leg. R.Sz. Żarnowiec, 1 August 1982, 1 male; 25 April 1984, 1 male, leg. R.Sz.; 4 July 1989, 1 male. Southern Baltic Lakelands. Babi Dół near Kartuzy, 13 May 1989, 3 males, leg. R.Sz. Gdynia Bernadowo, Łęg nad Swelinią Reserve, 3 September 2011, light trap, 1 male, leg. M.G.K.; 10 September 2011, light trap, 1 male, leg. M.G.K. Lake Otalżyno near Kartuzy, 5 July 1979, 1 female, leg. R.Sz. Miastko near Bytów, 28 July 1990, 1 male, leg. A.W. EASTERN Baltic LaKelands. Krzywe near Suwałki, Apiaceae, 1 July 2005, net, 2 males, leg. P.D. Łopuchowo near Suwałki, 29 May 2006, net, 1 male, leg. P.D. Słupie near Suwałki, near River Czarna Hańcza, peat bog, 24 May 2006, net, 1 male, leg. P.D. CenTRAL Poland Lowlands. Czeszów near Wrocław, gravel pit, 30 August 2007, net, 7 males, 2 females, leg. P.D. PoDLASIE. Kunicha near Augustów, Anthriscus sylvestris (L.) HofFM., 7 June 1985, 1 female, leg. J.K. PoLESIE. Libiszów near Włodawa, 19/20 May 1964, at light, 1 male, leg. Z.B. 


\section{Distribution and biology}

Norway, Sweden, Russia (Karelia, Leningrad Oblast, North Ossetia), Estonia, Lithuania, Great Britain, Poland, Germany, the Netherlands, Belgium, Czech Republic, France, Switzerland, Austria, Hungary, Romania, Ukraine (Crimea), Bulgaria, Andorra, Spain, Georgia, Azerbaijan, Afghanistan, Iran, China, Japan, Algeria, Egypt, Yemen (DOMINIAK \& SZADZIEWSKI 2010).

Preimaginal stages of $D$. modesta are found in the littoral zone of lakes, in ponds, mud, swamps, peat-bogs as well as in saline habitats (THIENEMANN 1915, MAYER 1934a, ThienEmanN 1950, SzADZIEWSKi 1986, PRzhiboro 1999, Chandler et al. 2008). SZADZIEWSKI (1983) included this species among the haloxenes. Final-instar larvae of D. modesta are known to spin tubular cases (THIENEMANN 1915).

Dasyhelea modesta is listed from numerous localities in Poland, where adults were collected from April to September.

Discussion

Because the type materials of $D$. modesta and most of its synonyms do not exist, interpretation of this species follows those by ZILAHI-SEBESS (1940) and REMM (1962) (SZADZIEWSKI 1986).

The armature of the female genitalia and palpus in D. modesta resemble those in D. wirthi ClastriER, a species known from the Afrotropical Region. These two species differ in the colouration of the antennal segments, which are entirely dark in $D$. modesta and bicoloured in $D$. wirthi. It is very probable that these two names are conspecific.

The records of $D$. modesta from Finland (HACKMAN 1980), Italy (BOORMAN et al. 1995) and Denmark (PETERSEN \& ACHIM 2001) are treated as doubtful (DOMINIAK \& SZADZIEWSKI 2010).

\section{Dasyhelea notata GOETGHEBUER, 1920}

\section{(Fig. 59)}

Dasyhelea notata GOETGHeBuer, 1920: 47 (description, in key - male, female, figure - female, Belgium); KIEFFER 1925c: 65 (description - male, female, figure - female); GOETGHEBUER 1934: 34 (description, in key - male, female, figure - female); SZADZIEWSKI 1986: 40 (description and figure - male, female, syn.: = semistriata, = sziladyi, Belgium, Poland); DelÉCOLLE \& RieB 1990: 184 (France); SzADZIEWSKi 1998: 103 (Switzerland); SzADZIEWSKi 2001: 273 (Poland); TóthovÁ et al. 2004a: 309 (Czech Republic); DominiaK \& SzADZIEwski 2006: 5 (Poland); TóthovÁ \& KNOZ 2006: 106 (comments, Czech Republic); DominiaK et al. 2007b: 4 (Poland); DominiAK \& SzADZIEWSKI 2010: 23 (syn.: = semistriata, = sziladyi, Algeria, Finland, North Korea, Romania, Sweden).

Dasyhelea semistriata GoetgheBuer, 1921: 176 (description and figure - male, Belgium); KiefFER 1925c: 68 (description - male); GOETGHEBUER 1934: 35 (description and figure, in key - male); SAHUQuillo HerRÁiz \& Gil Collado 1982: 747 (Spain).

Dasyhelea sziladyi ZILAHI-SEBESS, 1936a: 42 (description - male, female, figure - female, Hungary); ZILAHI-SEBESS 1940: 54 (description - male, female, figure - male, Hungary); REMM 1962: 111 
(description, figure - male, Estonia); REMM 1966: 58 (Lithuania); REMM 1967: 14 (Georgia); REMM 1969: 207 (in key, figure - male); REMM 1971: 198 (Russia: Ussuri Land); REMM 1973b: 354 (Hungary); REMM 1979: 49 (Estonia).

\section{Diagnosis}

Posterior margin of male sternite 9 with a pair of short, slightly divergent and horn-like projections. Posterior process distinctly stout mesally.

\section{Description}

Male. AR 0.92-0.99 $(n=10)$. Length of third palpal segment 84-110 $\mu \mathrm{m}(\mathrm{n}=11), \mathrm{PR}_{\text {(III) }}$ 4.94-6.11 $(\mathrm{n}=11)$. Colour of scutellum varies from yellow to brown; scutellum bears 7-8 bristles. Wing length $1.00-1.20 \mathrm{~mm}(\mathrm{n}=11)$, CR 0.50-0.55 $(\mathrm{n}=11)$. Genitalia (Fig. 59). Posterior margin of sternite 9 with a pair of horn-like projections, slightly divergent and quite distant one from another. Gonostylus slender, evenly curved. Parameres asymmetrical. Posterior process fused to right basal arm, distinctly swollen mesally and tapering distally. Middle part of this extension very often weakly sclerotized. Apicolateral processes of aedeagus flame-shaped or if scrolled then visible as a thin lamella.

Female. Anterior margin of subgenital plate rectangular. Spermatheca single, ovoid, with short neck, dimension 42-54x36 $\mu \mathrm{m}$ (SZADZIEWSKI 1986).

\section{Material examined $(n=14)$}

Poland: Southern Baltic Coastlands. Gdańsk, forests, 16 June 1980, 1 male. Eastern BALTiC LAKELANDS. Słupie near Suwałki, near River Czarna Hańcza, riparian forest, 5 July 2005, net, 1 male, leg. P.D. Michnowce near Sejny, 15 August 2007, net, 1 male, leg. P.D. Rosochaty Róg near Suwałki, 3 July 2005, net, 1 male, leg. P.D. PodLASIE. Kunicha near Augustów, Pimpinella sp., 21 August 1985, 1 male, leg. J.K.; Angelica sylvestris L., 24 August 1985, 1 male, leg. J.K. Pogorzelce near Białowieża, forest margin, 4 June 1981, 1 male, leg. R.Sz. SudETY MTS. MTS. Sosnówka Dolna near Jelenia Góra, 10 August 1982, 1 male, leg. R.Sz.; meadows near fish ponds, 3 August 1982, 1 male, leg. R.Sz. Central Western Carpathians. Czarny Dunajec near Nowy Targ, Baligówka peat bog, 28 June 2006, net, 3 males, leg. P.D. EASTERn BeSKID MTs. Brzegi Górne near Ustrzyki Górne, Apiaceae, 11 July 2005, net, 1 male, leg. P.D. Muczne near Ustrzyki Górne, riparian forest near Litmirz Reserve, 13 July 2005, net, 1 male, leg. P.D.

\section{Distribution and biology}

Sweden, Finland, Estonia, Lithuania, Poland, Belgium, France, Switzerland, Czech Republic, Hungary, Romania, Spain, Georgia, Russia (Ussuri Land), North Korea, Algeria (DOMINIAK \& SZADZIEWSKI 2010).

In Poland this species has been found in a few localities in the north of the country and in the mountains, where adults were collected from June to August. Dasyhelea notata is known to visit umbelliferous flowers.

\section{Discussion}

The records from Great Britain (EDWARDS 1926, 1929), Iceland (GOETGHEBUER \& LiNDROTH 1931), Poland (MAYER 1934b) and Germany (HAVELKA 1976a), which were based on females, are considered doubtful. The paper by ZILAHI-SEBESS (1940) mentions 
D. notata (as D. semistriata) from Slovakia, but according to the illustration of the male genitalia, this record is more likely to refer to D. thienemanni SPĂTARU \& DAMIANGEORGESCU (see below).

Type specimens of $D$. notata are deposited in the collection of the Royal Belgian Institute of Natural Sciences in Brussels (SZADZIEWSKI 1986).

\section{Dasyhelea lucida REMM, 1968}

(Figs 55, 60)

Dasyhelea lucida Remm in Remm \& Zhogolev, 1968: 831 (description and figure - male, female, Azerbaijan, Georgia (i.a. Abkhazia) - locus typicus, Ukraine: Crimea); REMM 1967: 14 (Azerbaijan, Georgia); REMM 1979: 49 (Estonia); SZADZIEWSKI 1991: 106 (Poland); BERNOTIENE 2002: 290 (Lithuania); CHANDLER et al. 2008: 84 (figure - male, comments, Great Britain); DOMINIAK \& SZADZIEWSKI 2010: 18 (Algeria, Spain, Pakistan, Romania).

\section{Diagnosis}

Posterior margin of male sternite 9 bent inwards. Posterior process stout, sometimes with slightly recurved tip. Aedeagus with a pair of short and finger-like median projections.
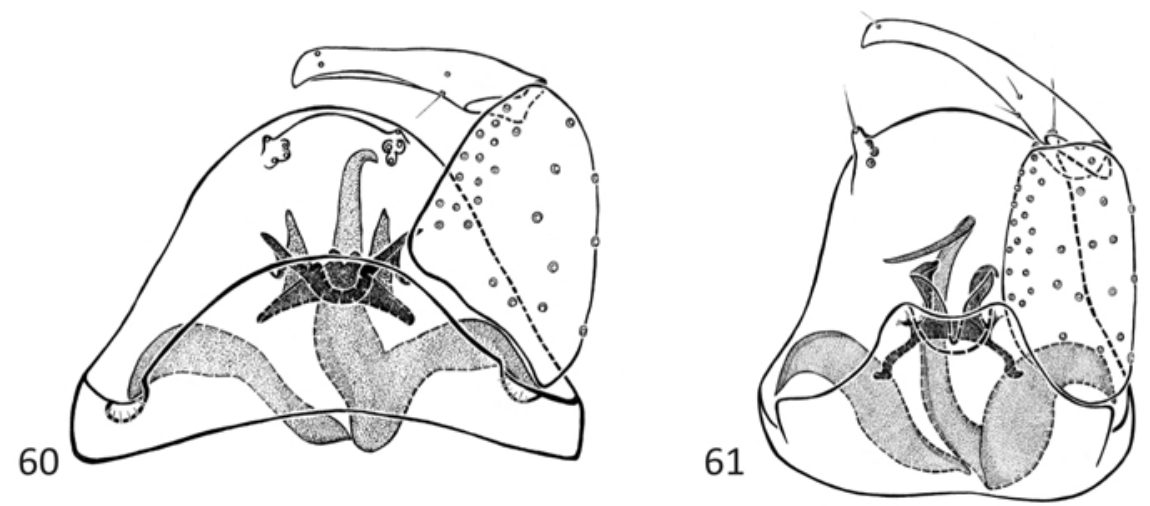

Figs 60-61. Male genitalia: 60 - Dasyhelea lucida Remm, 61 - D. stellata Remm.

\section{Description}

Male. AR 0.94-1.03 $(\mathrm{n}=10)$. Third palpal segment 58-76 $\mu \mathrm{m}$ long $(\mathrm{n}=11), \mathrm{PR}_{(\mathrm{III})} 3.94-$ $5.14(\mathrm{n}=11)$. Scutellum varies from entirely yellow to entirely dark, armed with 7-8 bristles and a few additional setae. Radial cell $r_{1}$ slit-like (Fig. 55); wing length 0.71-1.02 mm ( $\mathrm{n}=13)$, CR 0.45-0.49 $(\mathrm{n}=13)$. Genitalia (Fig. 60). Apical part of sternite 9 bent inwards, fused to aedeagus by horn-like posterior projections. Gonostylus slender, slightly arched to nearly straight. Parameres asymmetrical. Posterior process fused to right basal arm, stout, sometimes with slightly recurved tip. Basal arch of aedeagus low. One pair of conical submedian projections and one pair of median finger-like projections present. 
Female. Subgenital plate arch-shaped, with conical extension on anterior margin. Spermatheca subspherical, dimension 64x52 $\mu \mathrm{m}$ (REMM \& ZHOGOLEV 1968).

Material examined $(n=4)$

Poland: EAstern Baltic Lakelands. Bachanowo near Suwałki, 1 July 1993, 1 male, leg. J.K. PodLASIE. Kunicha near Augustów, Anthriscus sylvestris (L.) HofFM., 7 June 1985, 1 male, leg. J.K. Sudety MTs. MTs. Sosnówka Dolna near Jelenia Góra, 10 August 1982, 1 male, leg. R.Sz. CenTRAL Western CarPathians. Szafranówka in Pieniny Mts., 24 June 2003, net, 1 male, leg. M.G.K.

Distribution and biology

Great Britain, Estonia, Lithuania, Poland, Ukraine (the Eastern Carpathians, Crimea), Romania, Spain (Iberian Peninsula, Canary Is.), Georgia (i.a. Abkhazia), Azerbaijan, Pakistan, Algeria (DOMINIAK \& SZADZIEWSKI 2010).

Dasyhelea lucida is known from the north-eastern regions of Poland and the Sudety Mountains. The flight season in our country is from June to August; the biting midges can be found on white umbelliferous flowers as well as on spurges (DOMINIAK \& SZADZIEWSKI 2010).

\section{Discussion}

The German record of D. lucida (HAVELKA \& AGUILAR 1999) is considered doubtful (DOMINIAK \& SZADZIEWSKI 2010).

Type specimens of this species were deposited in the Tartu Ülikooli Loodusmuuseum in Estonia (REMM \& ZHOGOLEV 1968), but at present there are only two empty pins with "paratypus" labels in the collection.

\section{Dasyhelea stellata REMM, 1968}

(Figs 49, 50, 61)

Dasyhelea stellata REMm in REMM \& ZHOGOLEV, 1968: 831 (description and figure - male, female, Ukraine: Crimea, Russia: North Ossetia - locus typicus); REMM 1967: 14 (Russia: North Ossetia); DeléCOlle \& Rieb 1990: 184 (France); DeléCOlle et al. 1991: 256 (France); TÓTHOVÁ \& KNOZ 2006: 106 (Czech Republic); SzADZIEWSKI 2007: 64 (Poland); CHANDLER et al. 2008: 86 (figure male, comments, Great Britain); DOMINIAK \& SZADZIEWSKI 2010: (distribution).

\section{Diagnosis}

Posterior margin of male sternite 9 bent inwards. Posterior process slender, markedly twisted in distal part. Submedian projections of aedeagus elongate and strongly expanded distally.

\section{Description}

Male. AR 0.98-1.11 $(\mathrm{n}=6)$. Frontal sclerite elliptical or club-shaped, with lateral lobes (Fig. 49). Clypeus entire, with 6-8 setae (Fig. 50). Length of third palpal segment 95-117 $\mu \mathrm{m}(\mathrm{n}=6), \mathrm{PR}_{(\mathrm{III})}$ 5.27-6.93 $(\mathrm{n}=6)$. Scutellum dark yellow to slightly brown, bearing 8-10 bristles. Wing length 1.12-1.34 mm $(\mathrm{n}=6)$, CR 0.47-0.51 $(\mathrm{n}=6)$. Genitalia (Fig. 61). 
Posterior margin of sternite 9 bent inwards, fused to aedeagus with a pair of horn-like, slightly divergent processes. Gonostylus slender and evenly curved. Parameres asymmetrical. Posterior process long, fused to right basal arm, markedly twisted in distal part. Aedeagus arched. Submedian projections of aedeagus elongate, strongly expanded in apical half.

Female. Subgenital plate elongate, with conical notum. Spermatheca single, subspherical, length $75 \mu \mathrm{m}$ (REMM \& ZHOGOLEV 1968)

Material examined $(n=6)$

Poland: Southern BALtic CoAStLAnds. Sopot, beach, 3 June 1999, net, 1 male, leg. E.K. Southern Baltic LaKelands. Jaźwiny near Wałcz, on the River Korytnica, 12 June 2004, net, 2 males, leg. P.D.; 16 June 2004, net, 1 male, leg. P.D. Szwecja near Wałcz, on the River Piława, 20 June 2004, net, 1 male, leg. P.D. Silesian-Kraków Upland. Dolina Będkowska near Kraków, 10 July 1984, 1 male, leg. W.K.

Distribution and biology

Great Britain, Poland, France, Czech Republic, Ukraine (Crimea), Russia (North Ossetia) (DOMINIAK \& SZADZIEWSKI 2010).

The species is known from four localities in Poland. Adults of this biting midge were collected in June and July, mainly from plants growing near rivers. In Scotland a single male specimen of D. stellata was found in a saltmarsh (CHANDLER et al. 2008).

Discussion

The record from Germany (HAVELKA \& AGUILAR 1999) is considered invalid.

The type specimens of $D$. stellata are deposited in the Tartu Ülikooli Loodusmuuseum in Estonia.

\section{Dasyhelea glukhovae BRODSKAYA, 1996}

(Fig. 62)

Dasyhelea glukhovae BRODSKAYA, 1996: 193 (description and figure - larva, pupa, male, female, breeding site - small pit with moss, Carex and aquatic plants on a river bank, Kyrgyzstan); DOMINIAK \& SZADZIEWSKI 2010: 16 (Poland, Ukraine).

\section{Diagnosis}

Apex of gonostylus distinctly swollen. Posterior margin of male sternite 9 with short, slightly divergent finger-like processes. Submedian projections of aedeagus expanded distally, cornet-like.

\section{Description}

Male. AR 1.00-1.06 $(\mathrm{n}=3)$. Third palpal segment 89-93 $\mu \mathrm{m}$ long $(\mathrm{n}=3), \mathrm{PR}_{(\text {(III) }} 5.47$ $(\mathrm{n}=2)$. Scutellum pale or dark, with 8 bristles. Wing length $1.02-1.06 \mathrm{~mm}(\mathrm{n}=2), \mathrm{CR}$ 0.48-0.49 ( $\mathrm{n}=2$ ). Genitalia (Fig. 62). Posterior margin of sternite 9 extended, with short 
and slightly divergent finger-like processes. Gonostylus evenly curved, very broad in apical half. Parameres asymmetrical and stout, posterior process and right basal arm fused. Posterior process broad in the middle part and tapering distally. Aedeagus high arched, with a pair of distally expanded, cornet-like submedian projections.

Female. Subgenital plate arch-shaped, with irregular anterior border. Spermatheca small (length ca. $65 \mu \mathrm{m}$ ), subspherical, lacking neck (BRODSKAYA 1996).

Material examined $(n=2)$

Poland: EAstern Baltic LaKelands. Bachanowo near Suwałki, 1 July 1993, 1 male, leg. J.K. SudETy MTS. Sosnówka Dolna near Jelenia Góra, net, 4 August 1982, 1 male, leg. R.Sz.

\section{Distribution and biology}

Poland, Ukraine, Kyrgyzstan (DOMINIAK \& SzADZIEWSKI 2010).

The larvae of D. glukhovae were found in a small pit with moss, Carex and aquatic plants on a mountain river bank in Kyrgyzstan (BRODSKAYA 1996). In Poland the species is known from only two localities. Adult males were collected at the beginning of July and August.

\section{Discussion}

Males of D. glukhovae and D. thienemanni have very similar gonostyles. These two species differ in the shape of the submedian projections of sternite 9 and the armature of the aedeagus.

Type specimens of D. glukhovae are deposited in the collection of the Zoological Institute of the Russian Academy of Sciences in St. Petersburg (BRODSKAYA 1996).

62

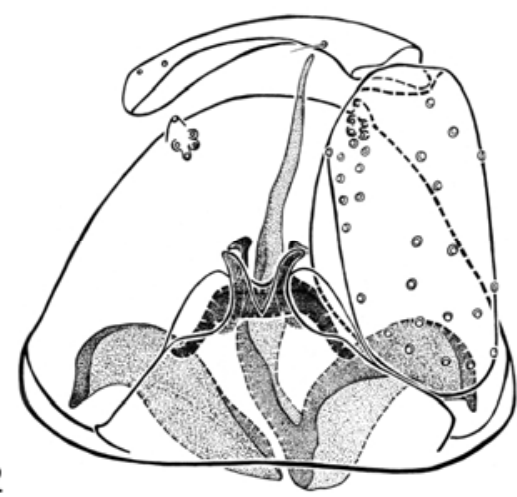

63

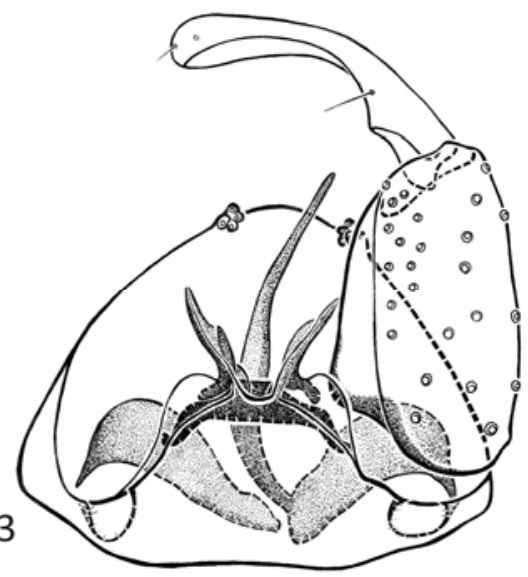

Figs 62-63. Male genitalia: 62 - Dasyhelea glukhovae BrodSKAYA, 63 - D. thienemanni SPĂTARU \& DAMIAN-Georgescu. 


\section{Dasyhelea thienemanni SPĂTARU \& DAMIAN-GEORGESCU, 1970}

(Figs 48, 51, 53, 63)

Dasyhelea thienemanni SPĂTARU \& DAMIAN-GEORGESCU, 1970: 425 (description and figure - larva, pupa, male, female, breeding site - spring and puddle, Romania); SZADZIEWSKI 1986: 42 (= notata sensu ReMm, Bulgaria, Poland); DominiaK \& SZADZIEWSKI 2010: 26 (distribution, = notata sensu REMM, = semistriata sensu ZILAHI-SEBESS).

Dasyhelea notata: REMM 1962: 111 (description, figure - male, Estonia); REMM 1966: 58 (Lithuania); REMm 1967: 14 (Azerbaijan, Georgia); REMM 1973b: 354 (Hungary); ReMm 1979: 49 (Estonia). Nec D. notata GoetGHeBuer, 1920.

Dasyhelea semistriata: ZILAHI-SEBESS 1940: 52 (description and figure, in key - male, Slovakia). Nec D. semistriata GOETGHEBUER, 1921.

\section{Diagnosis}

Apex of gonostylus bulbous. Male sternite 9 with very long posterior projections. Submedian projections of aedeagus sharp.

\section{Description}

Male. Flagellomeres 10-12 with two rows of setae (Fig. 51); AR 0.93-1.10 $(\mathrm{n}=7)$. Third palpal segment 78-105 $\mu \mathrm{m}$ long $(\mathrm{n}=8), \mathrm{PR}_{(\mathrm{III})} 4.94-6.56(\mathrm{n}=8)$. Transverse suture well visible (Fig. 48). Scutellum usually dark, with ca 8 bristles. Wing with two radial cells (Fig. 53); wing length 0.91-1.15 mm $(\mathrm{n}=7)$, CR 0.46-0.48 $(\mathrm{n}=7)$. Genitalia (Fig. 63). Posterior margin of sternite 9 extended, with a pair of long and sharp projections. Gonostylus arched, with swollen apex. Parameres asymmetrical. Posterior process fused to right basal arm, fairly stout mesally and tapering to a point. Basal arch of aedeagus low. Submedian projections of aedeagus sharp.

Female. Spermatheca with neck, subspherical (SPĂTARU \& DAMIAN-GEORGESCU 1970).

Material examined $(\mathbf{n}=\mathbf{8})$

Poland: Southern Baltic Lakelands. Głusk near Wałcz, riparian forest, $53^{\circ} 04.727^{\prime} \mathrm{N}$ $15^{\circ} 55.607 ' \mathrm{E}, 14 \mathrm{~m}$ below sea level, 18 June 2005, net, 1 male, leg. P.D. Jaźwiny near Wałcz, on the River Korytnica, 12 June 2004, net, 1 male, leg. P.D. Załom near Wałcz, riparian forest near the Delta Płocicznej Reserve, 19 June 2005, net, 1 male, leg. P.D. EASTERn BAltic LAKELANDS. Poszeszupie near Suwałki, Chaerophyllum sp., 3 July 1993, 3 males, leg. J.K. Wiatrołuża Pierwsza near Suwałki, riparian forest on the River Wiatrołuża, 2 July 2005, net, 1 male, leg. P.D. CENTRAL WeStern CARPATHIANS. Czarny Dunajec near Nowy Targ, Baligówka peat bog, 28 June 2006, net, 1 male, leg. P.D.

\section{Distribution and biology}

Estonia, Lithuania, Poland, Slovakia, Hungary, Romania, Bulgaria, Georgia, Azerbaijan (DOMINIAK \& SZADZIEWSKI 2010).

Preimaginal stages of $D$. thienemanni were collected in a spring and the puddle around it (SPĂTARU \& DAMIAN-GEORGESCU 1970).

In Poland adults of this species have been reported in June and July, mainly in riparian forest. $D$. thienemanni is known from a few localities in the northern part of the country and from a peat bog in the Orawa-Nowy Targ Basin. 


\section{Discussion}

Because the original description of a female of $D$. thienemanni is inadequate (SPĂTARU \& DAMIAN-GEORGESCU 1970), only the records based on males are currently treated as valid. Thus, the German report (HAVELKA 1976a) was omitted in the summary of the geographical distribution of this species. The records from Latvia and Moldova (REMM 1988) are also considered doubtful.

This species was discovered 30 years before its description. D. thienemanni was mentioned for the first time from Slovakia under the name D. semistriata (ZILAHI-SEBESS 1940). In the papers by REMM $(1962,1966,1967,1973 \mathrm{~b}, 1979,1988)$ it was listed as D. notata.

\section{subgenus Prokempia}

Prokempia KIEFFER, 1913a: 163, 179. Type species Dasyhelea ornaticornis KIEFFER, 1913a: 180 (designated by WIRTH, 1973: 358).

\section{Diagnosis}

Frontal sclerite broad, elliptical or subspherical, with lateral ribbon-like borders and distinctly sclerotized in the centre part. Last flagellomere without apical prolongation. Clypeus composed of two separate sclerites. All palpal segments fairly short. Thorax without transverse suture. Wing with one radial cell.

Male. Apicolateral processes of tergite 9 usually minute. Posterior margin of sternite 9 elongate, with pincer-like projection. Parameres asymmetrical, posterior process often fused to the right basal arm.

Female. Antennal segments short, subspherical, slightly reticulated. Notum of subgenital plate usually barely visible, spatula-like, subcircular or heart-shaped. Rami often with a pair of sharp, well-sclerotized caudomedian projections. Spermatheca single, ovoid, with short neck.

\section{Discussion}

Most of the descriptions of females of this subgenus are based on inadequate characters. It appears that only close examination of the subgenital plate enables valid identification to species level. Both rami and the caudomedian processes are usually well-sclerotized, but the shape of the notum becomes visible only when the subgenital plate is dissected.

All biting midges of the subgenus Prokempia have wings with only one radial cell. However, a poorly defined radial cell $\mathrm{r}_{1}$ can occur in some female specimens.

So far, three species of Prokempia have been reported from Poland. Other European species of this subgenus are D. alonensis (STROBL), D. biunguis KIEFFER, D. decoratissima (Strobl), D. erici Havelka, D. lutea Remm and D. taurica Remm. Among them, $D$. biunguis has the widest distribution, and it is very probable that this species will be found in Poland. 


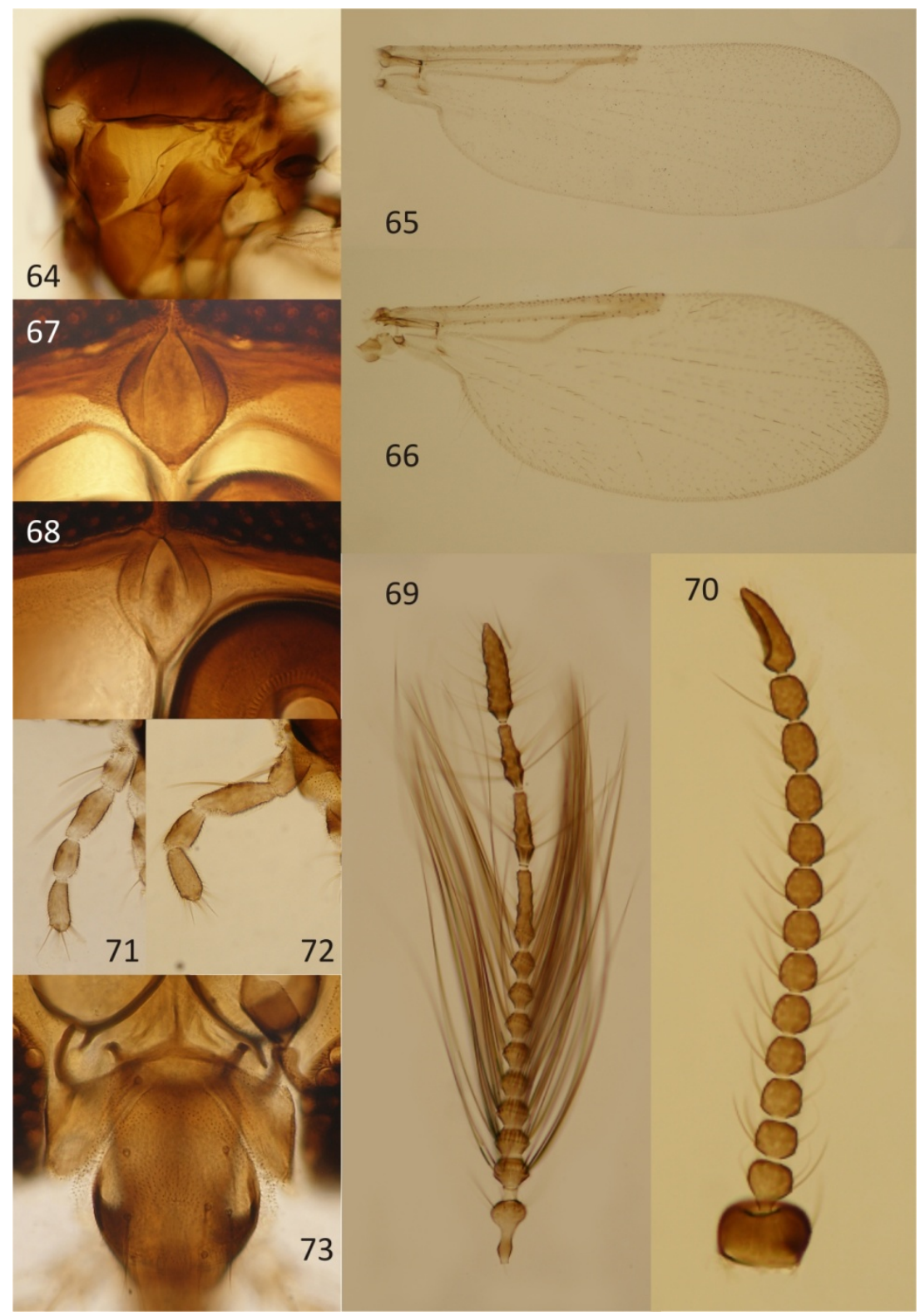

Figs 64-73. Dasyhelea bilobata KIEFFER, male $(65,71)$, female $(64,66,67,70,72,73)$, D. flaviventris (Goetghebuer), male $(68,69)$.

64 - thorax, 65, 66 - wing, 67, 68 - frontal sclerite, 69, 70 - antenna, 71, 72 - palpus, 73 - clypeus. 


\section{Key to species}

1. Apicolateral processes of male tergite 9 prominent; posterior process long and markedly twisted (Fig. 74). Female subgenital plate quite well visible, with spatula-shaped notum (Fig. 76)

bilobata

-. Apicolateral processes of male tergite 9 minute; posterior process short and rather straight (Fig. 77, 78). Female subgenital plate very weakly sclerotized, with rounded notum .

.2

2. Gonostylus with simple apex; submedian processes of aedeagus S-shaped (Fig. 77) .dampfi

-. Gonostylus with divided apex; submedian processes of aedeagus broad, funnel-shaped (Fig. 78)

flaviventris

\section{Dasyhelea bilobata KIEFFER, 1915}

(Figs 3, 10, 12, 13, 18, 19, 64, 65, 66, 67, 70, 71, 72, 73, 74, 75, 76)

Dasyhelea bilobata KiefFER, 1915: 65 (description - male, France); GoetghebUER 1934: 27 (description, in key - male); DOMINIAK \& SZADZIEWSKI 2010: 8 (syn.: = luteiventris $=$ spiralis).

Prokempia bilobata: KIEFFER 1925c: 71 (description and figure - male).

Dasyhelea luteiventris GoETGHeBUER, 1934: 90 (description and figure - female, Austria); SZADZIEWSKI 1986: 49 (description and figure - female, syn.: = spiralis, Poland); DELÉCOLLE et al. 1991: 256 (France); HAGAN et al. 2000: 471 (Norway); DelÉcolle 2002: 28 (syn.: = spiralis, Andorra); DominiaK \& SZADZIEWSKI 2006: 6 (breeding site - peat bogs, Poland); KNOZ et al. 2004: 84 (Czech Republic); ChANDleR et al. 2008: 87 (figure - male, comments, Great Britain).

Dasyhelea spiralis REMM, 1966: 60 (description and figure - male, female, Lithuania - locus typicus, Estonia, Russia: Leningrad Oblast); REMM 1967: 21 (Georgia); HAVELKA 1976a: 76 (Germany); HAVELKA 1976b: 227 (description and figure - male); REMM 1979: 50 (Estonia); PrZHibOro 1999: 133 (breeding site - lake littoral zone, Russia: Karelia); PrZHIBOro 2005: 167 (breeding site - water margin zone in lake, Russia: Karelia).

\section{Diagnosis}

Posterior margin of male sternite 9 with pincer-like projection. Apicolateral processes of tergite 9 prominent. Gonostylus hook-like, very stout in basal part and strongly tapering towards apex. Posterior process long, distinctly twisted. Female subgenital plate elongate, with spatula-like notum.

\section{Description}

Male. AR 0.86-1.03 $(\mathrm{n}=10)$. Palpal segment 3 cylindrical (Fig. 10, 71), 38-48 $\mu \mathrm{m}$ long $(\mathrm{n}=12), \mathrm{PR}_{(\mathrm{III})} 2.41-3.14(\mathrm{n}=12)$. Scutellum yellow or dark, bearing 6 bristles. Wing (Fig. 65) 0.81-1.02 mm long $(\mathrm{n}=11)$, CR 0.44-0.48 $(\mathrm{n}=11)$. Genitalia (Fig. 74). 
Apicolateral processes of tergite 9 prominent, cylindrical, with slightly extended apex. Posterior margin of sternite 9 with a pair of small triangle submedian projections and long, pincer-like median prolongation. Base of the latter with additional lobate protuberance. Gonostylus single, hook-shaped, with very stout basal part and distinctly narrow apex. Parameres asymmetrical, divided. Left basal arm longer than right one. Posterior process long and twisted, covered with tiny hairs in apical half. Aedeagus high arched, $\mathrm{H}$-shaped. Submedian processes slightly convergent.

Female. Antennal segments short, subspherical or subcylindrical (Fig. 3, 70); AR 0.74$0.92(\mathrm{n}=7)$. Frontal sclerite elliptical with lateral borders (Fig. 13, 67). Clypeus composed of two sclerites, short, with 7-8 setae (Figs 12, 73). Palpal segment 3 cylindrical (Fig. 72), 38-50 $\mu \mathrm{m}$ long $(\mathrm{n}=7), \mathrm{PR}_{\text {(III) }}$ 2.29-2.78 $(\mathrm{n}=7)$. Transverse suture absent (Fig. 64). Scutellum yellow, bearing 6 bristles. Wing with one radial cell (Fig. 66); wing length 0.77$0.84 \mathrm{~mm}(\mathrm{n}=5)$, CR 0.48-0.50 $(\mathrm{n}=5)$. Genitalia (Figs 18, 19, 75, 76). Subgenital plate elongate, notum spatula-like, weakly sclerotized. Spermatheca ovoid, with short neck, length 59-67 $\mu \mathrm{m}(\mathrm{n}=6)$ and width $43-50 \mu \mathrm{m}(\mathrm{n}=6)$.
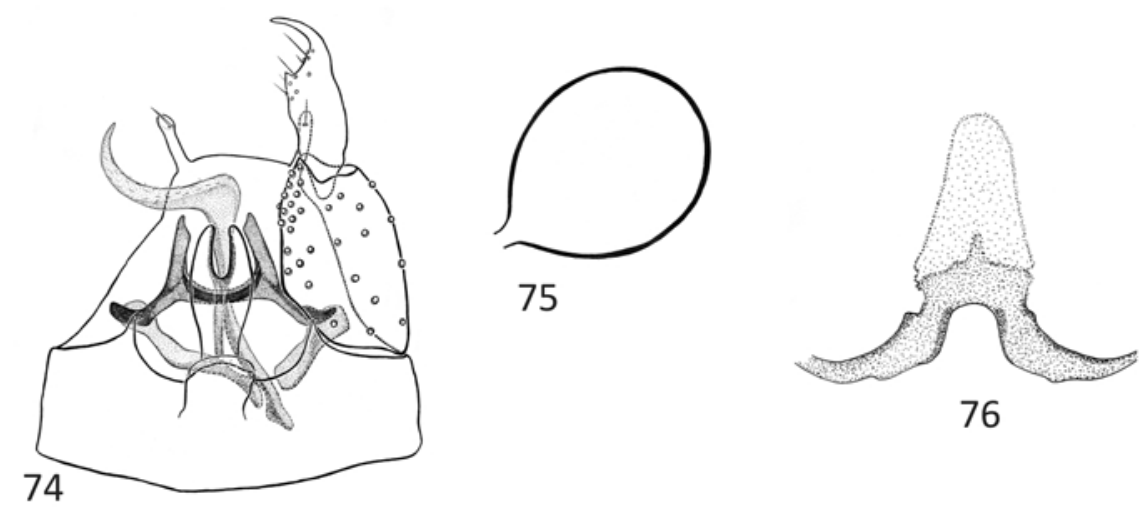

Figs 74-76. Dasyhelea bilobata KIEFFER: 74 - male genitalia, 75 - spermatheca, 76 - female subgenital plate.

\section{Material examined $(n=21)$}

Poland: Southern Baltic Lakelands. Babi Dół near Kartuzy, Chaerophyllum hirsutum L., 15 June 1985, 1 male, 1 female, leg. J.K. Mirachowo near Kartuzy, Apiaceae, 9 June 2005, net, 7 males, leg. P.D. Żakowo near Sulęczyno, 1 June 1992, 1 male. EASTERn Baltic LaKelands. Giżycko, Apiaceae, 2 June 1981, 2 females, leg. R.Sz. Solanka near Kętrzyn, near lake, Apiaceae, 15 August 1980, 2 males, leg. R.Sz. PodLASIE. Kunicha near Augustów, Angelica sylvestris L., 24 August 1985, 1 female, leg. J.K. Popiołówka near Białystok, peat bog, 4 June 1981, ex cult., 1 female, leg. R.Sz. Sztabin near Augustów, Cicuta virosa L., 25 August 1985, net, 1 male, leg. J.K. SudETy MTS. Sosnówka Dolna near Jelenia Góra, 4 August 1982, net, 1 male, leg. R.Sz.; 3 August 
1982, 1 female, leg. R.Sz. Central MąopolsKa Upland. Święta Katarzyna near Kielce, meadow on the Czarna Woda River, 50 55.158'N 2054.136'E, 276 m, 27 July 2008, net, 1 male, leg. P.D. Central Western Carpathians. Dolina Strążyska in the Tatra Mts., Apiaceae, 19 July 1993, 1 male, leg. J.K.

\section{Distribution and biology}

Norway, Estonia, Russia (Karelia, Leningrad Oblast), Lithuania, Great Britain, Germany, Poland, Czech Republic, France, Austria, Andorra, Georgia (DomINIAK \& SZADZIEWSKI 2010).

The larvae of $D$. bilobata are known to inhabit peat bogs and the littoral zone of lakes, where they live among mosses and liverworts. In Poland and Russia preimaginal stages were collected in June (PrZhiboro 2005, DominiaK \& SzADZIEWSKi 2006). D. bilobata has been reported from numerous localities in Poland. Adult males and females visit umbelliferous flowers.

\section{Discussion}

The original description (KIEFFER 1915) of Dasyhelea bilobata does not provide detailed information on the sampling site and the number of specimens examined. Male or males were collected in the Dauphiné, a former province in south-east France. Type specimens of $D$. bilobata probably do not exist. The holotype of $D$. luteiventris is housed in the Naturhistorisches Museum Wien (SzADZIEWSKI 1986), while the types of D. spiralis are deposited in the Tartu Ülikooli Loodusmuuseum (REMM 1966).

\section{Dasyhelea dampfi KIEFFER, 1925}

(Fig. 77)

Dasyhelea dampfi KIEFFER, 1925a [March 1925]: 150 (description, in key - female, Estonia); GoetGHeBuer 1934: 32 (description, in key - female); Remm 1962: 127 (description and figure male, syn.: = turfacea, = ?estonica, Estonia); REMM 1966: 60 (Lithuania); REMM 1969: 208 (in key, figure - male); REMm 1979: 50 (syn.: = turfacea, Estonia); DomINIAK \& SzADZIEWSKI 2006: 5 (Poland); TÓтHOvÁ \& KNOZ 2006: 106 (Czech Republic); DominiaK \& SzADZIEwSKI 2010: 11 (syn.: = estonica, distribution).

Dasyhelea turfacea KIEFFER, 1925a: 151 (description, in key - male, female, figure - male, Estonia); GOETGHEBUER 1934: 36 (description, in key - male, female).

Dasyhelea estonica KIEFFER, 1925b [December 1925]: 409 (description - male, Estonia).

\section{Diagnosis}

Posterior margin on male sternite 9 with pincer-like extension. Gonostylus rather short, stout in basal part, with simple apex. Aedeagus arched with two S-shaped submedian projections.

\section{Description}

Male. AR 0.90-0.95 $(\mathrm{n}=3)$. Frontal sclerite and clypeus typical of the subgenus, the latter with 8-9 setae. Length of third palpal segment 37-40 $\mu \mathrm{m}(\mathrm{n}=3), \mathrm{PR}_{(\mathrm{III})} 2.64-2.86(\mathrm{n}=$ 
3). Scutellum dark yellow, bearing 6 bristles. Wing length $0.80-0.94 \mathrm{~mm}(\mathrm{n}=3), \mathrm{CR} 0.42$ $0.43(\mathrm{n}=3)$. Genitalia (Fig. 77). Posterior margin of sternite 9 extended, with two, slightly divergent submedian processes and a single, pincer-like median projection. Gonostylus short, broader in its basal half, not divided in the apical half. Parameres asymmetrical. Posterior process moderately long and fused to right basal arm. Aedeagus evenly curved, with a pair of S-shaped submedian projections.

Female. Imprecise descriptions.

Material examined $(\mathbf{n}=\mathbf{3})$

Poland: Eastern Baltic Lakelands. Słupie near Suwałki, near the River Czarna Hańcza, peat bog, 5 July 2005, net, 1 male, leg. P.D. Central Western Carpathians. Czarny Dunajec near Nowy Targ, Baligówka peat bog, 28 June 2006, net, 1 male, leg. P.D. Chyżne near Nowy Targ, Puścizna Jasiowska peat bog, 29 June 2006, net, 1 male, leg. P.D.

Distribution and biology

Estonia, Lithuania, Poland, Czech Republic (DOMINIAK \& SZADZIEWSKI 2010).

Adult males of this species were collected during the summer months, on peat bogs in north-eastern and southern Poland.

\section{Discussion}

Dasyhelea dampfi was mentioned from Germany by HAVELKA \& AGUILAR (1999). This record, for which no locality data is given, as well as those from Hungary (ZILAHI-SEBESS 1940), Spain (SAHUquillo HerráIz \& Gil Collado 1982) and Russia (Krivosheina 1957), which were based on females, are here considered doubtful.

The type specimens of $D$. dampfi and its junior synonyms probably do not exist.
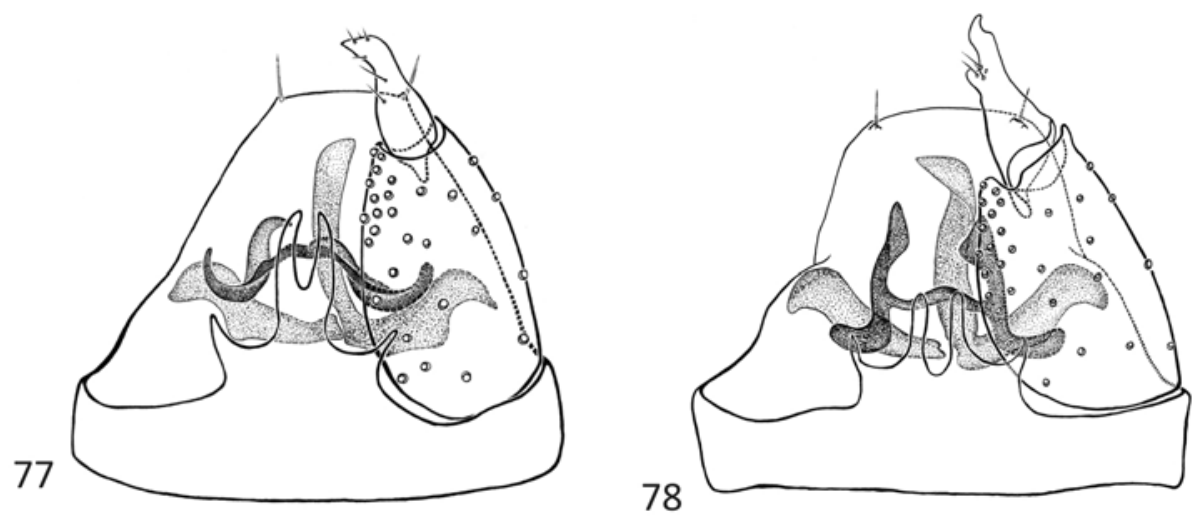

Figs 77-78. Male genitalia: 77 - Dasyhelea dampfi KIEFFER, 78 - D. flaviventris (GOETGHEBUER). 


\section{Dasyhelea flaviventris (GOETGHEBUER, 1910)}

(Figs 4, 5, 68, 69, 78)

Culicoides flaviventris GOETGHEBUER, 1910: 96 (description - female, Belgium).

Dasyhelea flaviventris: KIEFFER 1919: 49 (in key); GOETGHEBUER 1920: 41 (description and figure, in key - male, female, Belgium); GoETGHEBUER 1933a: 365 (syn.: = halobia); GOETGHEBUER 1934: 28 (description, in key - male, female, syn.: = scutellatus WINNERTZ nec MEIGEN, = halobia); THIENEMANN 1950: 167 (syn.: = halobia, breeding site - moss on wood log in water margin zone, Austria); RemM 1962: 127 (description - male, female, figure - male, Estonia); REMm 1966: 60 (Lithuania); REMM 1967: 22 (Azerbaijan, Georgia); REMM 1969: 208 (in key, figure - male); REMM 1973b: 355 (Hungary); HAVELKA 1976a: 62 (Germany); HAVELKA 1976b: 225 (description and figure - male, female, syn.: = scutellata WINNERTZ nec MEIGEN, = halobia); HAVELKA 1978: 176 (Germany, syn.: = scutellata WinNERTZ nec MEIGEN, = halobia); REMM 1979: 50 (Estonia); SAhuquillo Herráiz \& Gil Collado 1982: 748 (Spain); Gil Collado \& Sahuquillo Herráiz 1983: 44 (in key - male, female); DeLÉCOlle \& RieB 1990: 184 (France); SzADZIEWSKI 1991: 106 (Poland); KNOZ 1997: 81 (Czech Republic); KNOZ 1998: 118 (Czech Republic); TóthovÁ et al. 2004a: 309 (Czech Republic); TóthovÁ et al. 2004b: 146 (Czech Republic); DominiAK et al. 2007b: 4 (Poland); DominiaK 2009: 200 (Poland); DominiaK \& SzADZIEwSKI 2010: 15 (comments on synonymy, biology and distribution, Algeria, Bulgaria, North Korea, Morocco, Romania).

Prokempia halobia Kieffer, 1924: 12 (description - male, female, breeding site - saline habitats, Germany).

Prokempia flaviventris: KIEFFER 1925c: 72 (description - male, female, figure - female).

\section{Diagnosis}

Posterior process of male sternite 9 relatively short, in the form of two finger-like appendages. Apex of gonostylus bilobate. Aedeagus H-shaped, with submedian projections apically somewhat expanded.

\section{Description}

Male. Antennal flagellomeres 10-12 with single row of long setae; AR 1.03-1.14 $(\mathrm{n}=14)$ (Figs 4, 5, 69). Frontal sclerite elliptical (Fig. 68). Palpal segment 3 cylindrical, 30-50 $\mu \mathrm{m}$ long $(\mathrm{n}=19), \mathrm{PR}_{(\mathrm{III})} 2.31-3.36(\mathrm{n}=17)$. Scutellum yellow or dark, with 6 bristles. Wing length 0.76-1.04 mm $(n=17)$, CR 0.42-0.46 $(n=17)$. Genitalia (Fig. 78). Posterior margin of sternite 9 with a pair of triangular submedian processes and relatively short posterior process. The latter in the form of two finger-like appendages. Gonostylus short, with bilobed and irregular apex. Parameres asymmetrical. Posterior process fused to right basal arm, moderately long and sometimes bent apically. Aedeagus H-shaped, with a pair of broad, funnel-shaped posterior projections.

Female. Imprecise descriptions.

Material examined $(\mathbf{n}=\mathbf{1 8})$

Poland: Southern Baltic Coastlands. Gdańsk Oliwa, soil in corral, 14 July 1979, ex cult., 1 male, leg. R.Sz. Żarnowiec, 25 April 1984, 1 male, leg. R.Sz. Southern BaLtic LaKelandS. 
Mirachowo near Kartuzy, Apiaceae, 9 June 2005, net, 1 male, leg. P.D. Mrowiniec near Tuchola, 30 July 1993, 1 male, leg. E.K. Miechucino near Kartuzy, Staniszewskie Błoto Reserve, sector 209i, peat bog, 17 August 2005, Barber trap, 1 male, leg. M.C. EASTERn BALTiC LAKELANDS. Olsztyn, Apiaceae, 5 August 1991, 2 males, leg. K.P. Piecki near Mragowo, by lake, 5 August 1988, 1 male, leg. R.Sz. Silec near Kętrzyn, wet-ground forest, 3-14 August 1979, net, 1 male, leg. R.Sz. SudETY MTS. Sosnówka Dolna near Jelenia Góra, 4 August 1982, net, 1 male, leg. R.Sz.; meadow near ponds, 3 August 1982, 1 male, leg. R.Sz. Central Western CARPathians. Dolina Strążyska in the Tatra Mts., Apiaceae, 19 July 1993, 1 male, leg. J.K. Przysłop Miętusi in the Tatra Mts., 21 July 1993, 1 male, leg. J.K. EASTERN BESKID MTs. Moczarne near Wetlina, 22 June 2004, net, 1 male, leg. M.G.K.

Other specimens

Belgium: Destelbergen, 21.5.14 [1914], Collection M. Goetghebuer, Ex-Typis, đ̂, 348, cf. Mem. Mus. roy. Hist. Nat. T. VIII, fasc. 3, p. 41, sec. Goetghebuer \& Lenz in E. Lindner Flieg. pal. Reg. iii, 13a, 1933, p. 28: D. (Prokempia) flaviventris Goetgh., Dasyhelea flaviventris Goetgh., dét. M. Goetghebühr 1920, [IRSNB]. Destelbergen, 21.5.14 [1914], Collection M. Goetghebuer, ExTypis, ㅇ, cf. Mem. Mus. roy. Hist. Nat. T. VIII, fasc. 3, p. 41, sec. Goetghebuer \& Lenz in E. Lindner Flieg. pal. Reg. iii, 13a, 1933, p. 28: D. (Prokempia) flaviventris Goetgh., Dasyhelea flaviventris Goetgh., dét. M. Goetghebühr 1920, [IRSNB]. Destelbergen, 21.5.14 [1914], Coll. et det. M. Goetghebuer, Ex-Typis, ㅇ, cf. Mem. Mus. roy. Hist. Nat. T. VIII, fasc. 3, p. 41, sec. Goetghebuer \& Lenz in E. Lindner Flieg. pal. Reg. iii, 13a, 1933, p. 28: D. (Prokempia) flaviventris Goetgh., Dasyhelea flaviventris Goetgh., dét. M. Goetghebühr 1920, [IRSNB]. Schelderode, 25.5.42 [1942], M. Goetghebuer, TYPE ㅇ, M. Goetghebuer, Coll. et det. M. Goetghebuer, Dasyhelea flaviventris Goetg., R.I.Sc.N.B. 18.073, [IRSNB].

Distribution and biology

Estonia, Lithuania, Germany, Poland, Belgium, Czech Republic, France, Austria, Hungary, Romania, Bulgaria, Spain, Georgia, Azerbaijan, North Korea, Morocco, Algeria (DOMINIAK \& SZADZIEWSKI 2010).

Larvae of $D$. flaviventris were found among mosses sprouting on a wet log on a lake margin (THIENEMANN 1950). They are also known to live in moist soil.

This species has been reported from several localities in northern and southern Poland. Adults of $D$. flaviventris were collected in April, June, July and August.

\section{Discussion}

GoetGHEBUER described the species from a single female collected in Ghent (French: Gand) on $7^{\text {th }}$ September 1910. Unfortunately, the type material has probably been lost. Only specimens from East Flanders (Destelbergen and Schelderode) were found in the collection of the IRSNB.

The records from Zaire (GOETGHEBUER 1933b) and Great Britain (EDWARDS 1926), based on females, are here considered doubtful. ZILAHI-SEBESS (1940) listed Dasyhelea flaviventris from Hungary. Although his description of the species is inadequate and no illustration of the male genitalia is provided either, this record can be treated as valid, because the occurrence of $D$. flaviventris in Hungary was later confirmed by REMM (1973b). 


\section{subgenus Pseudoculicoides}

Pseudoculicoides MALloch, 1915: 309. Type species Ceratopogon mutabilis CoQuILletT, 1901: 602 (by original designation).

\section{Diagnosis}

Frontal sclerite narrow, elongate, elliptical or rhombic, evenly sclerotized, sometimes with additional ribbon-like borders in apical half. Last antennal segment without apical prolongation. Clypeus composed of two sclerites, rarely entire with lateral lobes. Wing usually with one radial cell, exceptionally radial cell $r_{1}$ also present.

Male. Apicolateral processes of tergite 9 prominent. Posterior margin of sternite 9 extended, very often fused to aedeagus by well-sclerotized bridge. Gonostylus single or composed of two parts. Parameres symmetrical or asymmetrical.

Female. Subgenital plate usually rounded or reniform, with spherical, reniform or heartshaped opening, rarely arch-shaped or with elongate notum. Spermatheca with short neck, single, subspherical, ovoid or retort-shaped. Females of some species have very long cerci.

Discussion

All Dasyhelea of the mutabilis group (WIRTH 1952) and the borgmeieri group (WIRTH \& WAUGH 1976) belong to the subgenus Pseudoculicoides, as do some species classified by WAUGH \& WIRTH (1976) in the leptobranchia group. It is the most species-rich and at the same time a very diverse subgenus of Dasyhelea midges. Both the similarities and the unique features appear in various combinations among the males of the particular Pseudoculicoides species. Moreover, because the preimaginal stages and females are poorly known, the relationships between the species of this subgenus are still unclear.

To date, 33 species of Pseudoculicoides have been reported from Europe, 14 of which are known to occur in Poland.

\section{Key to species}

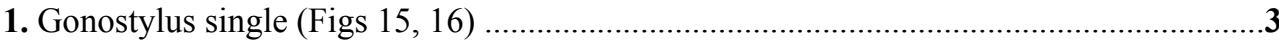

-. Gonostylus composed of two parts (Fig. 17) ....................................................................

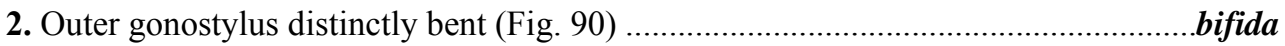

-. Outer gonostylus straight to slightly curved (Figs 17, 91) ........................................ furva

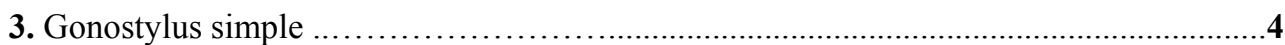

-. Gonostylus divided or with ventral finger-like projection at its base ...........................10

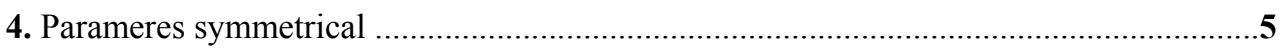

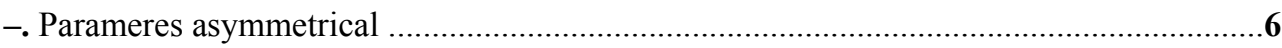

5. Parameres fused, posterior process short. Male sternite 9 conical, fused to aedeagus by conspicuous bridge (Figs 100, 101). Clypeus composed of two sclerites 
-. Parameres divided, posterior process long and slender. Male sternite 9 otherwise. Clypeus entire with lateral lobes

6. Male sternite 9 with rounded apex, joined with aedeagus with conspicuous bridge (Figs $104,105)$ .13

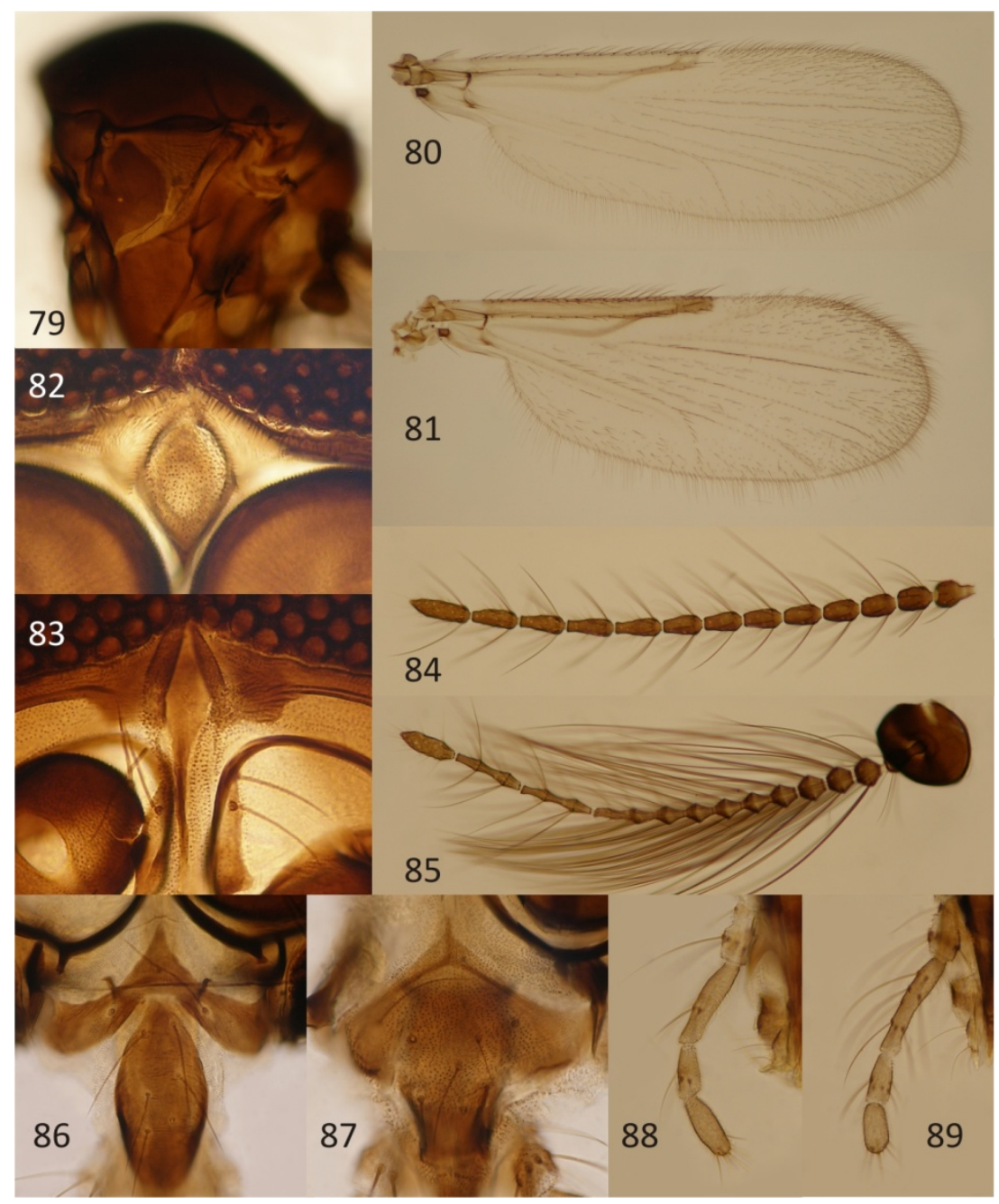

Figs 79-89. Dasyhelea arenivaga MACFIE, male (85, 89), D. bifida Zilahi-SeBeSS, male (80), D. corinneae Gosseries, male $(86,88)$, female $(81,83,84)$, D. flavoscutellata (ZETTERSTEDT), male (82), D. kyrenica ReMm, male $(79,87)$.

79 - thorax, 80, 81 - wing, 82, 83 - frontal sclerite, 84,85 - antenna, 86, 87 - clypeus, 88,89 palpus. 
-. Male sternite 9 otherwise, without bridge joining aedeagus with sternite, or if bridge present then apex of sternite 9 conical, very narrow (Fig. 96)

7. Male sternite 9 elongated distally, with divided or roof-like apex (Figs 92, 93) ...........8

-. Male sternite 9 rather short, lacking such projection 9

8. Male sternite 9 with divided apex. Posterior process fused to left basal arm (Fig. 92) calycata

-. Male sternite 9 with roof-like apex. Posterior process fused to right basal arm (Fig. 93)

corinneae

9. Submedian processes of aedeagus broad, C-shaped. Posterior margin of sternite 9 narrow, conical, fused to aedeagus by a bridge. Apex of gonostylus bulbous. Posterior process fused to left basal arm (Fig. 96) auli

-. Submedian processes of aedeagus pincer-like. Posterior margin of sternite 9 very broad, not joined to aedeagus. Apex of gonostylus slender. Posterior process fused to right basal arm (Fig. 97)

bicrenata

10. Gonostylus long, with ventral, finger-like projection at its base. Apicolateral processes of tergite 9 cornet-like. Posterior process slender, with distinctly bent apex (Fig. 98)

leptoclada

-. Gonostylus short, pincers-like. Apicolateral processes of tergite 9 conical. Posterior process broad, with expanded apex (Fig. 99) unguistyla

11. Median projection of aedeagus with truncated apex (Figs 15, 100) ................turanicola -. Median projection of aedeagus tapering to a point (Fig. 101) ..............................turficola

12. Male sternite 9 with two submedian, finger-like processes (Figs 16, 102)...........kyrenica

-. Male sternite 9 without such processes, concave (Fig. 103) similaris

13. Apicolateral processes of tergite 9 cylindrical, with slightly expanded, conical apex; seta located near apex of the processes (Fig. 104) arenivaga

-. Apicolateral processes of tergite 9 triangular, tapering sharply to a point; seta located in the middle of the processes (Fig. 105)

flavoscutellata

\section{Dasyhelea bifida ZILAHI-SEBESS, 1936}

(Figs 80, 90)

Dasyhelea fasciigera KIEFFER var. bifida, ZILAHI-SEBESS 1936a: 44 (description and figure - male, Hungary); ZILAHI-SEBESS 1940: 48 (description and figure - male, Hungary).

Dasyhelea bifida: Remm 1967: 21 (figure - male, Azerbaijan); Damian-Georgescu 1975: 97 (description and figure - male, Romania); NAVAI 1994: 364 (description and figure - male, Afghanistan); YU et al. 2006: 237 (description and figure - male, China); DOMINIAK et al. 2007a: 260 (figure - male, Israel); DOMINIAK \& SzADZIEWSKI 2010: 7 (comments on synonymy with D. furva REMM, 1967 and distribution, Poland, Ukraine).

Dasyhelea excellentis BORKENT, 1997: 91 (description and figure - male, female, USA: Hawaii). Syn. n. 


\section{Diagnosis}

Gonostylus in male genitalia composed of two elements. Outer gonostylus distinctly bent at midlength, inner gonostylus divided into two unequal and slightly divergent parts. Apicolateral processes of tergite 9 finger-like and close to one another.
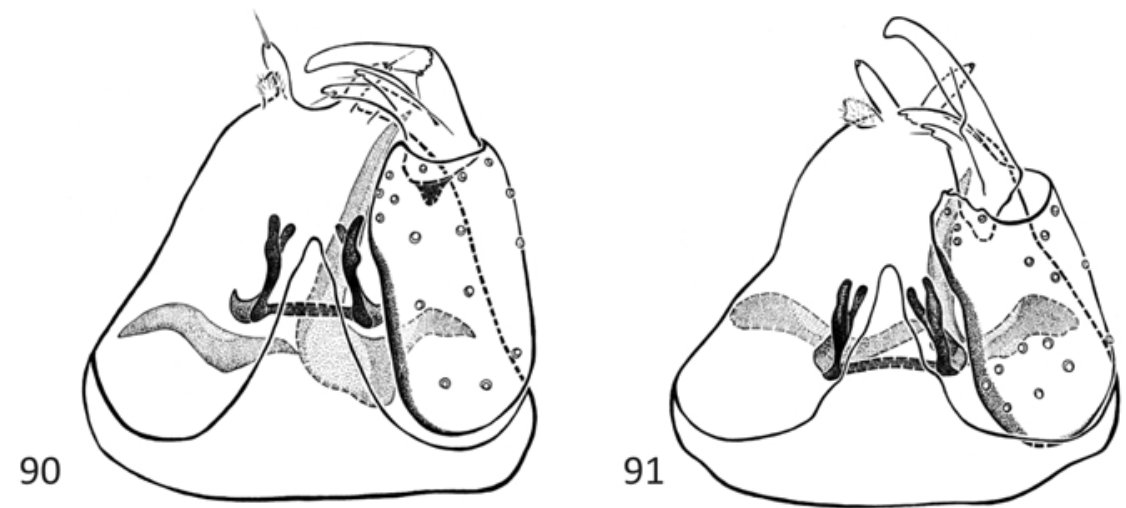

Figs 90-91. Male genitalia: 90 - Dasyhelea bifida ZILAHI-SEBESS, 91 - D. furva REMM.

\section{Description}

Male. AR 0.81-0.91 $(\mathrm{n}=10)$. Clypeus with 7-8 setae. Palpal segment 3 slender, 54-69 $\mu \mathrm{m}$ long $(\mathrm{n}=13), \mathrm{PR}_{(\mathrm{III})} 4.14-4.93(\mathrm{n}=12)$. Transverse suture absent. Scutellum yellow or dark, with 6 bristles and 1-2 setae. Wing with one radial cell (Fig. 80); wing length 0.78-0.91 mm $(n=12)$, CR 0.45-0.48 $(n=12)$. Genitalia (Fig. 90). Posterior margin of tergite 9 with a pair of finger-like, slightly divergent and adjoining apicolateral processes. Posterior margin of sternite 9 elongate, conical. Gonostylus composed of two parts. Outer gonostylus distinctly bent at midlength. Inner gonostylus divided into two wedge-shaped and unequal parts that do not fully overlap one another. Parameres asymmetrical. Posterior process broadly fused to right basal arm, stout basally and tapering to a point. Lateral projections of aedeagus forked in apical half.

Female. Notum broad and oblate, with elliptical lumen. Spermatheca single, spherical (BORKENT 1997).

Material examined $(\mathbf{n}=\mathbf{5})$

Poland: Southern Baltic Lakelands. Inowrocław Mątwy, Apiaceae, 19 August 2009, net, 4 males, leg. P.D. Central Western Carpathians. Krościenko on the River Dunajec, 12 August 1988, at light, 1 male, leg. Cz.M.

Distribution and biology

Poland, Hungary, Romania, Ukraine (Crimea), Azerbaijan, Israel, Afghanistan, China (DOMINIAK \& SZADZIEWSKi 2010).

In Poland D. bifida has been reported from saline habitats in the northern part of the 
country and one locality in the Carpathians. The adult males were collected in the middle of August, at light and from umbelliferous flowers.

\section{Discussion}

The unique shape of the male gonostyli distinguishes D. bifida from all other species of the fasciigera group. According to the original description (BORKENT 1997), the Hawaiian species $D$. excellentis has the same male genital armature. Moreover, other morphological characters, except antennal ratio (AR 0.51-0.57), seem to be identical to those in D. bifida. I consider these two taxa to be conspecific, so $D$. excellentis is proposed here as a new junior synonym of $D$. bifida. New synonymy.

In spite of the fact that both sexes of D. bifida were present in the materials from the Caucasus (REMM 1967), only the male genitalia of this species were illustrated. The first detailed description of the female was provided by BORKENT (1997).

The records from the Catalogue of Palaearctic Diptera (REMM 1988) and Checkliste der Dipteren Deutschlands (HAVELKa \& AGUILAR 1999), in which no precise localities are cited, have been omitted in the summary of the geographical distribution of D. bifida.

\section{Dasyhelea furva REMM, 1967}

(Figs 17, 91)

Dasyhelea furva REMM, 1967: 21 (description and figure - male, female, Estonia, Russia: North Ossetia - locus typicus); REMM 1979: 51 (Estonia); DominiaK \& SZADZIEWSKI 2010: 16 (comments on synonymy with D. bifida ZILAHI-SEBESS, 1936).

Dasyhelea bifida: BORKENT \& FORSTER 1986: 1283 (description and figure, in key - male, syn.: = furva, Canada); SzAdZIEWSKi 1991: 106 (Poland). Nec D. bifida Zilahi-SEBESS, 1936.

Dasyhelea fasciigera: REMM 1962 (partim): 129 (figure - male, Estonia). Nec D. fasciigera KiEFFER, 1925.

\section{Diagnosis}

Gonostylus in male genitalia bipartite. Outer gonostylus straight to slightly curved, inner gonostylus divided into two unequal but overlapping parts. Apicolateral processes of tergite 9 finger-like and close to one another.

\section{Description}

Male. AR 0.90-1.00 $(\mathrm{n}=3)$. Palpal segment 3 slender, length 58-70 $\mu \mathrm{m}(\mathrm{n}=3), \mathrm{PR}_{(\mathrm{III})}$ 3.62-4.38 ( $\mathrm{n}=3)$. Transverse suture absent. Wing length 0.72-0.91 mm $(\mathrm{n}=3)$, CR 0.42$0.48(\mathrm{n}=3)$. Genitalia (Figs 17, 91). Apicolateral processes of tergite 9 finger-like, divergent and close to one another. Posterior margin of sternite 9 conical. Gonostylus consists of outer and inner gonostylus, the former straight to slightly curved, the latter divided into two unequal parts that overlap one another. Parameres asymmetrical. Posterior process fused to right basal arm, tapering distally. Aedeagus with two lateral projections, similar to those in D. bifida. 
Female. Notum subcircular with heart-shaped opening. Spermatheca one, subspherical, length $68 \mu \mathrm{m}$ (REMM 1967).

Material examined $(\mathbf{n}=\mathbf{1})$

Poland: PODLASIE. Kunicha near Augustów, Angelica sylvestris L., 24 August 1984, 1 male, leg. J.K.

Distribution and biology

Estonia, Poland, Russia (North Ossetia), Canada (DOMINIAK \& SZADZIEWSKI 2010).

The record from north-east Poland is based on a single male collected from umbelliferous flowers at the end of August. In Estonia adults of $D$. furva were observed in June and August (REMM 1967).

\section{Dasyhelea calycata REMM, 1972}

(Figs 92, 124)

Dasyhelea calycata REMm, 1972: 74 (description and figure - male, female, Russia: Buryatia, Tuva locus typicus); REMm 1973a: 173 (Mongolia); PRZHIBORO \& BRODSKAYA 2006: 181 (breeding site - salt lake, Ukraine: Crimea); DOMINIAK \& SZADZIEWSKI 2010: 9 (comments on systematic position, biology and distribution, Bosnia and Herzegovina, Bulgaria).

Dasyhelea neobifurcata: SzADZIEWSKi 1983: 66 (Poland); CHANDLER et al. 2008: 88 (figure - male, comments, breeding site - saline habitats, Great Britain). Nec D. neobifurcata WIRTH, 1976

Dasyhelea bifurcata: REMM 1966: 60 (Lithuania); REMM 1967: 17 (Russia: North Ossetia); ReMm \& Zhogolev 1968: 832 (Ukraine: Crimea); REMM 1969: 208 (in key, figure - male); DAMIANGEORGESCU 1973: 451 (description and figure - male, Romania); REMM 1973b: 355 (Hungary); HAVELKA 1979: 65 (Spain). Nec D. bifurcata WIRTH, 1952.

Dasyhelea falvoscutellata: ZILAHI-SEBESS 1940: 49 (description - male, female, figure - male, syn.: = egens, $=$ flaviscapula, $=$ ?alonensis, $=$ halobia, $=$ heracleae, Slovakia, Hungary); MAYER 1959: 97 (figure - male, breeding site - saline habitats, Spain). Nec D. flavoscutellata (ZETTERSTEDT, 1850).

\section{Diagnosis}

Apex of posterior margin of male sternite 9 forked. Posterior process elongate, sickleshaped and fused to left basal arm.

\section{Description}

Male. AR 0.79-1.02 $(\mathrm{n}=10)$. Palpal segment 3 slender, 56-76 $\mu \mathrm{m}$ long $(\mathrm{n}=10), \mathrm{PR}_{(\mathrm{III})}$ 3.47-4.44 $(\mathrm{n}=10)$. Transverse suture absent. Scutellum with 6-8 bristles. Wing length 0.80 $1.04 \mathrm{~mm}(\mathrm{n}=10)$, CR 0.44-0.50 $(\mathrm{n}=10)$. Legs entirely dark. Genitalia (Fig. 92). Apicolateral processes of tergite 9 cylindrical and moderately long. Posterior margin of sternite 9 extended, its apical part forked. Gonostylus slightly curved. Parameres asymmetrical. Posterior process broadly fused to left basal arm fused, long and sickleshaped, with minute hair in distal half. Basal arch of aedeagus low. Apicolateral projections of aedeagus elongate, with slightly scrolled lateral margins and recurved tips.

Female. See below. 
Material examined $(n=651)$

Poland: Southern Baltic CoAstlands. Brzyno near Żarnowiec, Apiaceae, 2 June 1982, 1 male, leg. R.Sz. Gdańsk, Sobieszewska Is., Górki Wschodnie, saline mud, 5 May 1977, ex cult., 18 May 1977, 1 male, leg. R.Sz. Żarnowiec, mud from lake margin, 20 July 1982, ex cult., 1 male, leg. R.Sz. SOUTHERN BALtic LAKELANDS. Inowrocław Mątwy, saline habitat, 6 July 1973, net, 3 males, leg. R.Sz.; 3 August 1973, 11 males, leg. R.Sz.; 15 May 1975, 16 males, leg. R.Sz.; 4 August 1975, 4 males, leg. R.Sz.; 6 August 1975, 7 males, leg. R.Sz.; 25 April 2008, ex cult., 29 April - 20 May 2008, 7 pupae, 174 males, leg. P.D.; ex cult., 11-13 May 2008, 1 male, leg. P.D.; 19 August 2009, net, 74 males, leg. P.D.; ex cult., 8 September - 12 October 2009, 168 males, leg. P.D.; soil, 1 August 1973, ex cult., 1 male, leg. R.Sz.; Salicornietum, soil, 5 March - 15 April 1975, ex cult., 25 males, leg. R.Sz.; Salicornietum, 1 August 1974, 6 males, leg. R.Sz.; 10 August 1974, 1 male, leg. R.Sz.; 1 March 1975, ex cult., 6 males, leg. R.Sz.; 5 March - 15 April 1975, ex cult., 4 males, leg. R.Sz.; 15 May 1975, 3 males, leg. R.Sz.; 12 July 1975, 10:00 - 12:00, 65 males, leg. R.Sz.; mud, 1 August 1974, ex cult., 8 males, leg. R.Sz.; Salicornia europaea L., Triglochin sp., 15 May 1975, 8 males, leg. R.Sz.; Triglochin sp., 4 August 1975, 23 males, leg. R.Sz.; Festucetum, 15 May 1975, 3 males, leg. R.Sz.; 4 August 1976, 1 male, leg. R.Sz.; Apiaceae, 19 August 2009, net, 1 male, leg. P.D. Janikowo near Inowrocław, saline habitat, 14 May 1975, 8 males, leg. R.Sz.; Salicornietum, 14 May 1975, 4 male, leg. R.Sz.; saline habitat, soil, 14 May 1975, ex cult., 3 males, leg. R.Sz. Miastko near Bytów, 13 August 1991, 1 male, leg. A.W. EASTERn BAltic LaKelands. Aleksiejówka near Sejny, Lake Pomorze, 15 August 2007, net, 1 male, leg. P.D. Poszeszupie near Suwałki, Chaerophyllum sp., 3 July 1993, 1 male, leg. J.K. CENTRAL Poland Lowlands. Chylice near Warszawa, meadow, 27 May 1982, net, 1 male, IZ PAN; 16 September 1982, net, 1 male, IZ PAN. Młochów near Warszawa, 30 April - 13 May 1991, 1 male, IZ PAN. Puszcza Biała, sector 34f, 19 June - 3 July 1986, 1 male, IZ PAN. PodLASIE. Hamlet of Maruszka on Siemianówka Reservoir near Białystok, Apiaceae, 11 August 2005, net, 3 males, leg. P.D. Kunicha near Augustów, Cicuta virosa L., 18 August 1985, 1 male, leg. J.K. Sudety MTs. Przełęcz Łącznik in the Izerskie Mts., 26 August 2007, net, 1 male, leg. P.D. Sosnówka Dolna near Jelenia Góra, Apiaceae, 2 August 1982, 1 male, leg. R.Sz.

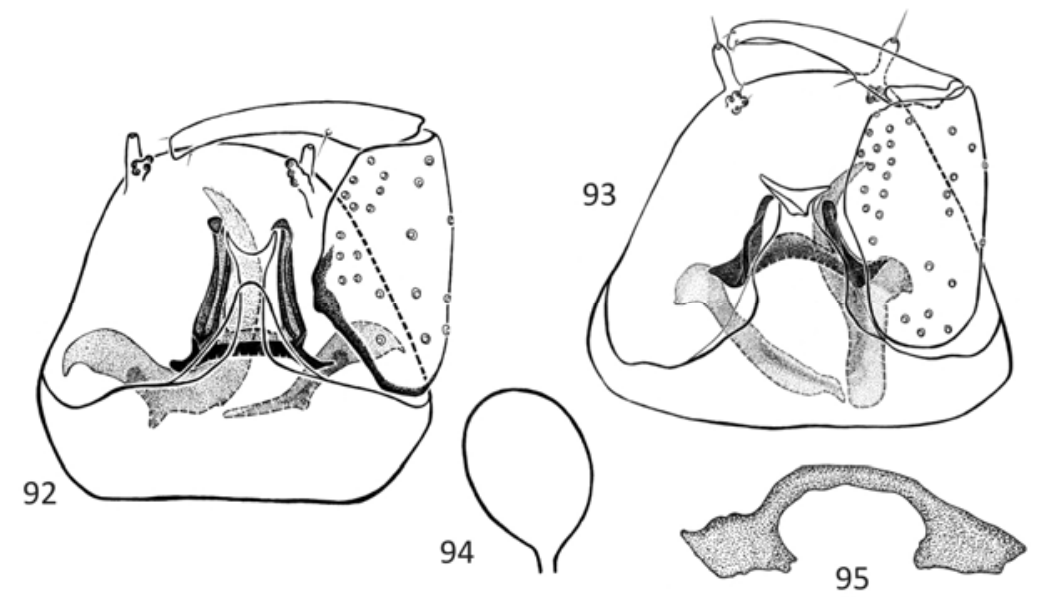

Figs 92-95. Dasyhelea calycata Remm (92) and D. corinneae Gosseries (93, 94, 95): 92, 93 - male genitalia, 94 - spermatheca, 95 - female subgenital plate. 


\section{Distribution and biology}

Great Britain, Lithuania, Poland, Slovakia, Bosnia and Herzegovina, Ukraine (Crimea), Romania, Bulgaria, Spain, Hungary, Russia (North Ossetia, Tuva, Buryatia), Mongolia (DOMINIAK \& SZADZIEWSKI 2010).

The larvae of $D$. calycata live in both fresh and salt water habitats. This halophilous species is known from many localities in Poland, and it is common especially on inland saline meadows. In Poland the flight period of adults is from May to September. Imagines reared from larvae emerged as early as April.

Discussion

It is very probable that the sexes of $D$. calycata have been incorrectly associated (see REMm 1974). The female with long cerci on sternite 10 belongs to another species, most likely to D. unguistyla ReMM, 1972 (DominiaK \& SZADZIEWSKI 2010).

It was impossible to find the type materials of $D$. calycata in the collection of the Tartu Ülikooli Loodusmuuseum.

\section{Dasyhelea corinneae GOSSERIES, 1991}

(Figs 81, 83, 84, 86, 88, 93, 94, 95)

Ceratopogon scutellatus MeIGEN, 1830: 262 (description - female, Europe).

Dasyhelea scutellata: KIEFFER 1919: 50 (in key); EDWARDS 1926: 402 (description, in key - male, Great Britain); Goetghebuer 1934: 28 (description - male); Zilahi-Zebess 1940: 52 (description - male, female, figure - male, Hungary); REMM 1967: 18 (Russia: North Ossetia); REMM 1971: 200 (figure - male, Russia: Ussuri Land); REMM 1973b: 355 (Hungary); WaugH \& WIRTH 1976: 236 (description and figure - male, female, Canada, USA).

Dasyhelea corinneae GosserIES, 1991: 42 (new name for C. scutellatus MEIGEN, 1830); CHANDLER 1998: 55 (syn.: = scutellata); DOMINIAK \& SZADZIEWSKI 2006: 6 (syn.: = scutellatus, Poland); DOMINIAK \& SZADZIEWSKI 2010: 10 (syn.: = chonetus, distribution, North Korea, Sweden, Ukraine). Dasyhelea chonetus Yu \& Zou in Yu et al. 2006: 279 (description and figure - male, China).

\section{Diagnosis}

Male sternite 9 produced distally, with roof-like apex. Posterior process broadly fused to right basal arm. Subgenital plate low-arched.

\section{Description}

Male. AR 0.96-0.98 $(\mathrm{n}=10)$. Clypeus with 7-11 setae (Fig. 86). Third palpal segment (Fig. 88) with sensilla capitata, 53-61 $\mu \mathrm{m}$ long $(\mathrm{n}=11), \operatorname{PR}_{(\mathrm{III})} 3.35-4.36(\mathrm{n}=11)$. Transverse suture short. Scutellum entirely yellow or with darker margins, bearing 6 bristles. Wing length 0.79-1.00 mm $(\mathrm{n}=11)$, CR 0.47-0.52 $(\mathrm{n}=11)$. Genitalia (Fig. 93). Apicolateral processes of tergite 9 long and finger-like. Posterior margin of sternite 9 markedly produced distally, with apical roof-like fold. Gonostylus long, nearly straight or slightly arched. Parameres asymmetrical. Posterior process curved, broadly fused to right 
basal arm. The latter very short. Aedeagus with a pair of moderately long lateral projections, tapering to a recurved tip.

Female. All flagellomeres elongate (Fig. 84), lacking reticulation; AR 0.78-0.81 $(\mathrm{n}=2)$. Frontal sclerite rhombic, with apicolateral borders (Fig. 83). Clypeus bearing 8-11 setae. Third palpal segment 44-50 $\mu \mathrm{m}$ long $(\mathrm{n}=2), \mathrm{PR}_{\text {(III) }} 2.59-2.78(\mathrm{n}=2)$. Transverse suture well visible. Scutellum yellow, with 6 bristles. Wing with one radial cell (Fig. 81), length $0.76-0.88 \mathrm{~mm}(\mathrm{n}=2)$, CR 0.50-0.51 ( $\mathrm{n}=2)$. Genitalia (Figs 94, 95). Subgenital plate lowarched. Spermatheca ovoid, with short neck, length $45 \mu \mathrm{m}(\mathrm{n}=1)$ and width $27-33 \mu \mathrm{m}(\mathrm{n}=2)$.

Material examined $(n=3)$

Poland: EASTERN BESKID MTs. Wołosate near Ustrzyki Górne, Wołosate Reserve, peat bog, 12 July 2005, net, 1 male, leg. P.D.

Other materials. Ukraine: Brusnicya near Chernivtsi, 48²1.449'N 2538,532'E, 170 m AMSL, 28 May 2008, net, 1 female, leg. P.D. USA: Maryland: Colesville, 15.VI.1977, W.W. Wirth, light trap, 1 female.

Distribution and biology

Great Britain, Sweden, Poland, Hungary, Ukraine, Russia (North Ossetia, Ussuri Land), China, North Korea, Canada, USA (DomINIAK \& SZADZIEWSKI 2010).

The record from Poland is that of a single male collected in the Bieszczady N.P.

Discussion

Some of the old reports of $D$. corinneae (STOR $\AA 1937$, ToKUNAGA 1940), as well as records based on females (SAHUQuillo HerRÁiz \& Gil Collado 1982) or those not provided with detailed locality data (Gosseries 1991, PETERSEN \& ACHIM 2001), are here regarded as doubtful.

\section{Dasyhelea auli REMM, 1962}

(Fig. 96)

Dasyhelea auli Remm, 1962: 123 (description and figure - male, female, Estonia); REMm 1979: 51 (Estonia); DominiaK \& SZADZIEWSKI 2010: 6 (Poland, Romania).

\section{Diagnosis}

Posterior margin of male sternite 9 narrow, joined with aedeagus by well-sclerotized bridge. Apex of gonostylus distinctly swollen. Posterior process broadly fused to left basal arm, elongate and bent in apical half. Submedian projections of aedeagus broad, C-shaped.

\section{Description}

Male. AR 0.96-0.97 $(\mathrm{n}=2)$. Clypeus with 7 long setae. Length of third palpal segment 47-49 $\mu \mathrm{m}(\mathrm{n}=2), \mathrm{PR}_{\text {(III) }}$ 3.77-3.92 $(\mathrm{n}=2)$. Transverse suture absent. Scutellum yellow or brownish, with 6 bristles. Wing length $0.73-0.82 \mathrm{~mm}(\mathrm{n}=2)$, CR 0.42-0.44 $(\mathrm{n}=2)$. Genitalia (Fig. 96). Apicolateral processes of tergite 9 long, slightly irregular at apex. Posterior margin of sternite 9 produced, conical, joined to the aedeagus by strongly 
sclerotized bridge. Gonostylus evenly arched, stout, bulbous in apical half. Parameres asymmetrical and fused. Posterior process broadly fused to left basal arm, elongate, markedly bent apically. Aedeagus low-arched, with single, conical median projection and a pair of stout, somewhat C-shaped submedian processes.

Female. Subgenital plate with ring-like notum. Spermatheca with short neck, subspherical (REMM 1962).

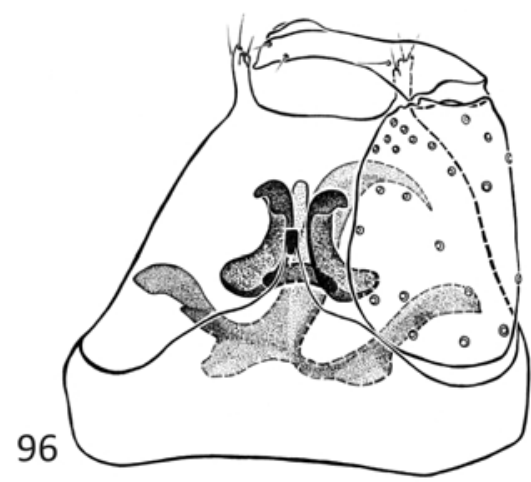

97

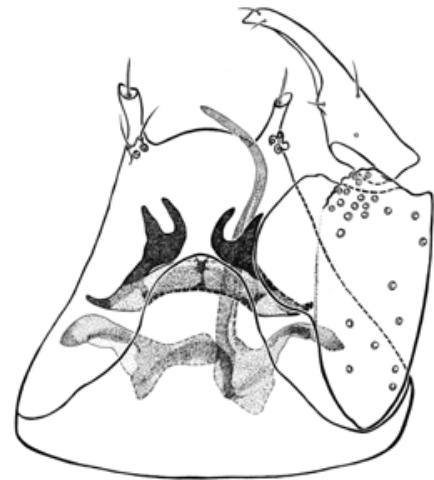

Figs 96-97. Male genitalia: 96 - Dasyhelea auli REMM, 97 - D. bicrenata KIEFFER.

Material examined $(\mathbf{n}=\mathbf{1})$

Poland: Eastern Baltic LaKelands. Poszeszupie near Suwałki, on Chaerophyllum sp., 3 July 1993, 1 male, leg. J.K.

Distribution and biology

Estonia, Poland, Romania (DOMINIAK \& SZADZIEWSKI 2010).

Dasyhelea auli is known from a single locality in north-east Poland. A single male was collected from umbelliferous flowers at the beginning of July. In the Eastern Carpathians in Romania this species was found in June (DOMINIAK \& SZADZIEWSKI 2010), while in Estonia adults were observed in late June - early July (REMM 1962).

\section{Discussion}

The male genitalia of $D$. auli are very distinctive. Like the species of the flavoscutellata group and the turficola group, there is a well-sclerotized bridge joining sternite 9 to the aedeagus. But the unique armature of the hypopygium separates this species from any other Palaearctic Pseudoculicoides. Dasyhelea auli most closely resembles the Nearctic species D. thomsenae WIRTH.

Dasyhelea auli was mentioned from Germany by HAVELKA \& AGUILAR (1999), and from Siberia by REMM (1988), but both of these records are treated as doubtful.

The holotype of $D$. auli is deposited in the collection of the Tartu Ülikooli Loodusmuuseum. 


\section{Dasyhelea bicrenata KIEFFER, 1923}

(Fig. 97)

Dasyhelea bicrenata KIEFFER, 1923: 668 (description and figure, in key - male, Algeria); GOETGHEBUER 1934: 31 (description and figure - male); REMM 1967: 19 (description and figure male, Georgia); SAhuquillo Herráiz \& Gil Collado 1982: 747 (Spain); Ramos Ramis \& SAhuquillo Herráiz 1983: 421 (description and figure - female, Spain); Delécolle \& Rieb 1993: 110 (Spain); SzAdZIEwSKi \& BORKENT 2003: 256 (Bulgaria, Poland); DominiaK \& SZADZIEWSKI 2010: 7 (distribution, Ukraine).

\section{Diagnosis}

Apicolateral processes of tergite 9 funnel-like. Gonostylus tapered distally. Posterior process slender and wavy, broadly fused to right basal arm. Submedian projections of aedeagus pincer-like.

\section{Description}

Male. AR 0.88-1.04 $(\mathrm{n}=10)$. Palpal segment 3 slender, 51-71 $\mu \mathrm{m}$ long $(\mathrm{n}=11), \mathrm{PR}_{(\mathrm{III})}$ 3.86-4.73 $(\mathrm{n}=11)$. Transverse suture absent. Scutellum yellow or dark, with 6-7 bristles and some fine setae in middle part. Wing length $0.67-0.86 \mathrm{~mm}(\mathrm{n}=11), \mathrm{CR} 0.39-0.47$ $(\mathrm{n}=11)$. Genitalia (Fig. 97). Apicolateral processes of tergite 9 elongate, with small apical prolongation, funnel-like. Posterior margin of sternite 9 extended, with slightly rounded or flattened apex. Gonostylus slender. Parameres asymmetrical, fused. Posterior process elongate, slightly wavy, broadly fused to right basal arm. Aedeagus with a pair of broad, pincer-like submedian projections.

Female. Spermatheca with short neck, small (RAMOS RAMIS \& SAHUQUILLO HERRÁIZ 1983).

Material examined $(n=4)$

Poland: Southern Baltic Coastlands. Puck, beach, 4 September 2001, net, 1 male, leg. E.K. Southern Baltic Lakelands. Starogard Gdański, Heracleum sibiricum L., 3 September 1987, 1 male, leg. J.K. EAStern MaŁopolska Upland. Brody Małe near Szczebrzeszyn, 10 July 2009, net, 1 male, leg. P.D. Kawęczyn near Szczebrzeszyn, 10 July 2009, net, 1 male, leg. P.D.

Distribution and biology

Poland, Ukraine (Crimea), Bulgaria, Georgia, Spain, Algeria (DOMINIAK \& SZADZIEWSKI 2010).

Up to now D. bicrenata has been reported from four localities in northern and southeastern Poland, where males of this species were found in July and September, i.a. on umbelliferous flowers.

\section{Discussion}

Dasyhelea bicrenata resembles the species of the fasciigera group (i.a. D. bifida, D. furva), but differs in having a single, not a divided gonostylus. In 1980 REMM described $D$. noctuabunda from Tajikistan, a species which seems to be very close to D. bicrenata. 
Dasyhelea bicrenata has been mentioned from Moldova and Siberia (REMm 1988), and also from Germany (HAVELKa \& AGUILAR 1999). These three records, devoid of locality data, have been omitted in the summary of the species' distribution.

\section{Dasyhelea leptoclada REMM, 1967}

(Fig. 98)

Dasyhelea leptoclada REMM, 1967: 19 (description and figure - male, Azerbaijan - locus typicus, Russia: Dagestan).

Dasyhelea leptoclada: GARCía SÁNCHEZ \& SAHUQuIllo HERRÁIZ 1986: 95 (description and figure female, Spain); SzADZIEWSKI 1983: 66 (Poland); DominiaK \& SZADZIEWSKI 2010: 18 (comments on biology and distribution).

\section{Diagnosis}

Apicolateral processes of male tergite 9 cornet-like. Gonostylus elongate, with short ventral projection at its base. Parameres fused. Posterior process slender, markedly curved in distal part.

\section{Description}

Male. AR 0.92-0.98 $(\mathrm{n}=11)$. Clypeus with 6-10 setae. Length of third palpal segment 57-65 $\mu \mathrm{m}(\mathrm{n}=11), \mathrm{PR}_{(\mathrm{III})} 3.59-4.57(\mathrm{n}=11)$. Transverse suture absent. Scutellum entirely yellow or with brownish margins, bearing 6 bristles. Wing length 0.83-0.94 $\mathrm{mm}(\mathrm{n}=10)$, CR 0.44-0.45 ( $\mathrm{n}=10)$. Genitalia (Fig. 98). Posterior margin of tergite 9 with two long and cornet-shaped apicolateral processes. Posterior margin of sternite 9 extended, conical. Gonostylus long, evenly arched, with short, finger-like process in basal part. Parameres asymmetrical and fused. Posterior process slender and distinctly bent apically, broadly fused to right basal arm. Basal arch of aedeagus low, apicolateral projections slender, with slightly recurved tips.

Female. Spermatheca with short neck, ovoid, length $69.3 \mu \mathrm{m}$ (GARCÍA SÁNCHEZ \& SAHUQUILLO HERRÁIz 1986).

Material examined $(n=43)$

Poland: SOUTHERn BALTiC COASTLANDS. Gdańsk, Sobieszewska Is., Górki Wschodnie, saline habitats, 10 June 1977, 3 males, leg. R.Sz. Southern BALtic LAKELANDS. Inowrocław Mątwy, saline habitat, Festucetum, 13 June 1975, 21 males, leg. R.Sz.; 4 August 1976, 1 male, leg. R.Sz.; Salicornietum, soil, 5 March - 15 April 1975, ex cult., 1 male, leg. R.Sz.; Salicornia europaea L., 4 August 1975, 1 male, leg. R.Sz.; Triglochin sp., 4 August 1975, 1 male, leg. R.Sz.; Apiaceae, 19 August 2009, net, 14 males, leg. P.D. Janikowo near Inowrocław, saline habitat, 14 May 1975, 1 male, leg. R.Sz.

Distribution and biology

Poland, Spain, Russia (Dagestan), Azerbaijan (DOMINIAK \& SZADZIEWSKI 2010).

Dasyhelea leptoclada is a halobiont. In Poland this species has been reported from saline habitats on the Gulf of Gdańsk and in inland saline meadows near Inowrocław. Adults of $D$. leptoclada are known to visit umbelliferous flowers. They have also been found on tamarisk flowers (REMM 1967). 


\section{Discussion}

The record from Germany (HAVELKA \& AGUILAR 1999) is treated here as doubtful.

The holotype of $D$. leptoclada is probably lost. The male specimen was deposited in the Tartu Ülikooli Loodusmuuseum in Estonia (REMM 1967), but recently only an empty pin, labelled holotypus, was found in the collection.
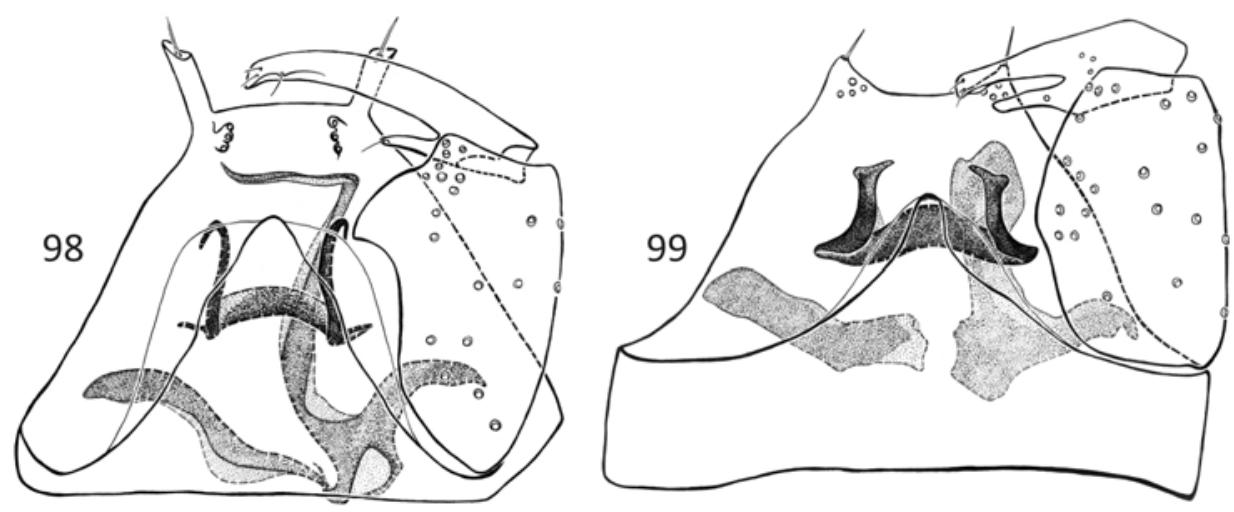

Figs 98-99. Male genitalia: 98 - Dasyhelea leptoclada Remm, 99 - D. unguistyla Remm.

\section{Dasyhelea unguistyla REMM, 1972}

(Fig. 99)

Dasyhelea unguistyla REMm, 1972: 79 (description and figure - male, Russia: Tuva, Zabaykalsky Krai, Yakutia - locus typicus); Remm 1973a: 173 (Mongolia).

Dasyhelea unguistyla: SzADZIEwSKI 1983: 66 (saline habitats, Poland); DOMINIAK \& SzADZIEwSKI 2010: 27 (comments on biology and distribution).

\section{Diagnosis}

Apicolateral processes of male tergite 9 conical. Gonostylus short and divided, pincerlike. Posterior process fused to right basal arm, expanded in the apical part, nearly clubshaped.

\section{Description}

Male. AR $0.91(\mathrm{n}=1)$. Clypeus elongate, with 8 setae. Palpal segment 3 cylindrical, $79 \mu \mathrm{m}$ long $(\mathrm{n}=1), \mathrm{PR}_{(\mathrm{III})} 4.16(\mathrm{n}=1)$. Scutellum dark, bearing 8 bristles. Wing length ca $0.96 \mathrm{~mm}(\mathrm{n}=1)$, CR ca $0.46(\mathrm{n}=1)$. Genitalia (Fig. 99). Apicolateral processes of tergite 9 short and conical, with one apical seta and a few additional small setae in basal half. Posterior margin of sternite 9 extended. Gonostylus stout, divided, in the form of crab pincers. Parameres asymmetrical. Posterior process broadly fused to right basal arm, nearly club-shaped, with apical part distinctly swollen. Aedeagus low arched, with two stout apicolateral projections. 
Female. See below.

Material examined $(\mathbf{n}=\mathbf{1})$

Poland: Southern Baltic Lakelands. Aleksandrów Kujawski, saline habitat, 4 August 1974, 1 male, leg. R.Sz.

Distribution and biology

Poland, Russia (Tuva, Zabaykalsky Krai, Yakutia), Mongolia (DOMINIAK \& SZADZIEWSKI 2010).

Dasyhelea unguistyla is a halobiont (SzADZIEWSKI 1983). The record from Poland is based on a single male collected in the first half of August from inland saline habitats near Inowrocław.

\section{Discussion}

A female described by REMM (1972) as D. calycata probably belongs to this species (DOMINIAK \& SZADZIEWSKI 2010).

\section{Dasyhelea turanicola REMM \& NAZARMUKHAMEDOV, 1969}

(Figs 15, 100)

Dasyhelea turanicola REMM \& NAZARMUKHAMEDOv, 1969: 56 (description and figure - male, female, Kazakhstan - locus typicus, Kyrgyzstan, Uzbekistan - in a burrow of great gerbil, Tajikistan, Turkmenistan); SZADZIEWSKI 1985a: 92 (description and figure - male, female, Germany); DominiaK \& SzADZIEwSKi 2008: 149 (Poland); DominiaK \& SzADZIEWSKi 2010: 26 (syn.: = serrata, distribution).

Dasyhelea serrata NAVAI, 1994: 383 (description and figure - male, Afghanistan).

\section{Diagnosis}

Posterior margin of male sternite 9 fused to aedeagus by well-sclerotized bridge. Gonostylus distinctly arched, with broad apex. Parameres fused. Median projection of aedeagus with truncated apex.

\section{Description}

Male. AR 0.80-0.84 $(n=2)$. Length of third palpal segment 49-59 $\mu \mathrm{m}(\mathrm{n}=3), \mathrm{PR}_{(\mathrm{III})}$ 3.28-4.08 $(\mathrm{n}=3)$. Scutellum yellow, with 6 bristles. Wing length $0.69-0.84 \mathrm{~mm}(\mathrm{n}=3)$, CR $0.42-0.43(\mathrm{n}=3)$. Genitalia (Figs 15, 100). Posterior margin of sternite 9 rounded, fused to aedeagus by conspicuous bridge. Gonostylus markedly arch-shaped, swollen in apical part. Parameres fused, nearly or completely symmetrical. Apicolateral projections of aedeagus long, bent mesally, loop-like, with recurved tips. Median process of aedeagus broad, with truncated apex.

Female. Subgenital plate in the form of a flattened ring with reniform lumen. Spermatheca with short neck, subspherical, large, length 94-100 $\mu \mathrm{m}$ and width $72 \mu \mathrm{m}$ (REMM \& NAZARMUKHAMEDOV 1969, SZADZIEWSKI 1985a). 

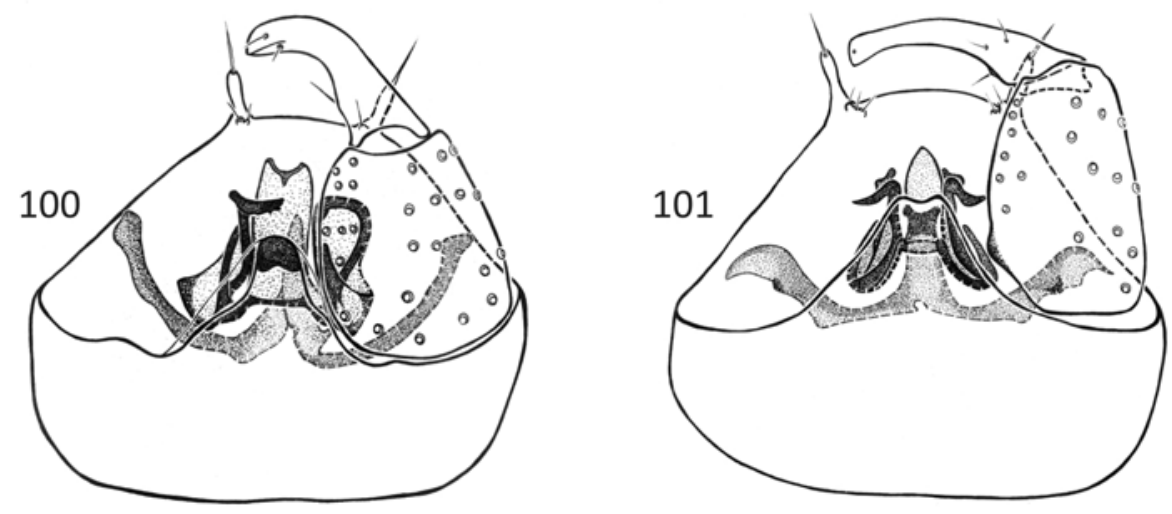

Figs 100-101. Male genitalia: 100 - Dasyhelea turanicola Remm \& NAZARMukhamedov, 101 - D. turficola KIEFFER.

Material examined $(\mathrm{n}=\mathbf{2})$

Poland: Central Poland Lowlands. Czeszów near Wrocław, gravel pit, 30 August 2007, net, 2 males, leg. P.D.

\section{Distribution and biology}

Germany, Poland, Kazakhstan, Uzbekistan, Turkmenistan, Kyrgyzstan, Tajikistan, Afghanistan (DOMINIAK \& SZADZIEWSKI 2010).

To date this species is known from a single locality in south-west Poland. Teneral males of $D$. turanicola were found at the end of August near a small gravel pit pond. In Asia adults of the species were collected from May to August, i.a. from tamarisk flowers (REMM \& NAZARMUKHAMEDOV 1969).

\section{Discussion}

The holotypes of D. turanicola were deposited in the Tartu Ülikooli Loodusmuuseum (REMM \& NAZARMUKHAMEDOV 1969), but only one paratype male has been found in the collection.

\section{Dasyhelea turficola KIEFFER, 1925}

(Fig. 101)

Dasyhelea turficola KIEFFER, 1925a: 152 (description and figure, in key - male, Estonia); KIEFFER 1929: 296 (description - female, on Bog Bilberry, Russia: Kaliningrad Oblast); GoETGHEBUER 1934: 36 (description, in key - male); REMM 1962: 124 (description - male, female, figure male, Estonia); REMm 1966: 60 (Lithuania); REMm 1967: 17 (Georgia); Remm 1969: 208 (in key, figure - male); REMM 1971: 200 (Russia: Ussuri Land); REMM 1979: 51 (Estonia); SzADZIEWSKI 1983: 66 (Poland); SZADZIEWSKI 1985a: 80 (description and figure - male, female, syn.: 
= grenieri, breeding site - saline habitats, moist soil, Algeria, Belgium, Poland, Spain: Canary Is.); KNOZ 1997: 81 (Czech Republic); KNOZ 1998: 118 (Czech Republic); DelÉCOLle 1998 (1999): 137 (Spain); KNOZ et al. 2004: 84 (Czech Republic); DOMINIAK \& SZADZIEWSKI 2006: 7 (breeding site - peat bogs, Poland); DominiaK et al. 2007a: 261 (figure - male, Israel); CHANDLER et al. 2008: 87 (= flavoscutellata sensu EDWARDS, figure - male, breeding site - saline habitats, Great Britain, Ireland); DOMINIAK \& SzADZIEWSKI 2010: 26 (= flavoscutellata sensu EDWARDS, syn.: = grenieri, $=$ malibui, Morocco, Sweden).

Dasyhelea flavoscutellata: EDWARDS 1926: 402 (syn.: = egens, Great Britain). Nec D. flavoscutellata (ZETTERSTEDT, 1850).

Dasyhelea grenieri ClASTRIER, 1966: 703 (description and figure - male, female, Tenerife).

Dasyhelea malibui Yu, 2008: 165 (description and figure - male, France).

\section{Diagnosis}

Bridge joining male sternite 9 with aedeagus conspicuous. Gonostylus slender. Parameres fused, symmetrical. Median projections of aedeagus tapering to a point, conical.

\section{Description}

Male. AR 0.93-1.08 $(\mathrm{n}=10)$. Palpal segment 3 slender, 59-80 $\mu \mathrm{m}$ long $(\mathrm{n}=10), \mathrm{PR}_{(\mathrm{III})}$ 4.12-5.50 $(\mathrm{n}=10)$. Transverse suture absent. Scutellum yellow or dark, with 6-9 bristles and a few smaller setae. Wing length 0.84-1.14 mm $(n=13)$, CR 0.43-0.48 $(n=13)$. Legs dark, especially knees, but tarsi paler. Genitalia (Fig. 7b). Posterior margin of sternite 9 extended, fused to aedeagus by conspicuous bridge. Gonostylus slightly curved, rather slender. Parameres fused and symmetrical, with short, triangular posterior process. Aedeagus with two pairs of apicolateral projections, both with recurved tips. Median process of aedeagus in the shape of an irregular cone.

Female. Subgenital plate with reniform opening. Spermatheca ovoid, with short neck, length 69-86 $\mu \mathrm{m}$, width 48-52 $\mu \mathrm{m}$ (REMM 1962, SZADZIEWSKI 1985a).

Material examined $(\mathbf{n}=\mathbf{3 7})$

Poland: Southern Baltic Coastlands. Gdańsk, on Gulf of Gdańsk, Triglochin sp., grasses, 25 July 1975, 1 male, leg. R.Sz. Gdańsk, Sobieszewska Is., Górki Wschodnie, saline soil, 5 May 1975, ex cult., 2 males, leg. R.Sz.; 25 May 1977, ex cult., 3 males, leg. R.Sz.; 29 May - 6 June 1977, ex cult., 3 males, leg. R.Sz.; 6 August 1977, ex cult., 1 male, leg. R.Sz.; beach, 13 May 2006, net, 1 male, leg. P.D. Gdańsk, Sobieszewska Is., Przegalina, soil, 19 June 1982, ex cult., 7 males, leg. G.O. Kiezmark, 16 July 1975, 1 male, B.S. Lisewo near Tczew, Apiaceae, 28 May 1982, 1 male, leg. R.Sz.; 28 June 1982, 2 males, leg. G.O. Puck, saline habitat, before rain, 22 August 2003, net, 1 male, leg. E.K. Wierzchucino near Żarnowiec, peat bog, 30 June 1983, ex cult., 1 male, leg. R.Sz. SOUthern BALtic LaKelands. Aleksandrów Kujawski, saline habitat, 16 July 1973, 2 males, leg. R.Sz. Inowrocław Mątwy, saline habitat, 25 April 2008, ex cult., 29 April 2008, 1 male, leg. P.D.; ex cult., 2 May 2008, 1 male, leg. P.D.; Salicornia europaea L., 4 August 1975, 1 male, leg. R.Sz. Toruń Barbarka, pine forest, 9 July 1981, 2 males, leg. R.Sz. EASTERN BALTiC LAKELANDS. Ełk, 13/14 August 1965, at light, 1 male, leg. Z.B. Kętrzyn, near pond, Apiaceae, 22 July 1981, 3 males, leg. R.Sz. Silec near Kętrzyn, woodland, Apiaceae, 16 August 1980, 1 male, leg. R.Sz. PodLASIE. Kunicha near Augustów, Pimpinella sp., 21 August 1985, 1 male, leg. J.K. 


\section{Distribution and biology}

Great Britain, Ireland, Sweden, Estonia, Lithuania, Russia (Kaliningrad Oblast, Ussuri Land), Poland, Czech Republic, Belgium, France, Spain (Iberian Peninsula, Canaries: Tenerife), Georgia, Morocco, Algeria, Israel (DominiAK \& SZADZIEWSKi 2010).

The larvae of $D$. turficola are known to inhabit peat bogs and moist soil along both fresh and salt water bodies. SZADZIEWSKI (1983) designated this species as a haloxene.

Dasyhelea turficola is probably widely distributed in Poland. It has been collected from several localities, from May to August. Adults visit umbelliferous flowers.

Discussion

The records of D. turficola from Germany (HAVELKA \& AGUILAR 1999) and the Netherlands (KNOZ \& BEUK 2002) are not included in the distribution data. This species was listed from Great Britain as D. flavoscutellata (EDWARDS 1926).

The type material of $D$. turficola is probably lost.

\section{Dasyhelea kyrenica REMM, 1972}

(Figs 16, 79, 87, 102)

Dasyhelea kyrenica Remm, 1972: 75 (description and figure - male, female, Russia: Buryatia); DOMINIAK \& SZADZIEWSKI 2008: 149 (Poland); DominiaK \& SZADZIEWSKi 2010: 17 (Romania).

\section{Diagnosis}

Clypeus entire. Posterior margin of sternite 9 in male genitalia with a pair of submedian, finger-like appendages. Gonostylus slightly swollen mesally. Parameres nearly symmetrical.
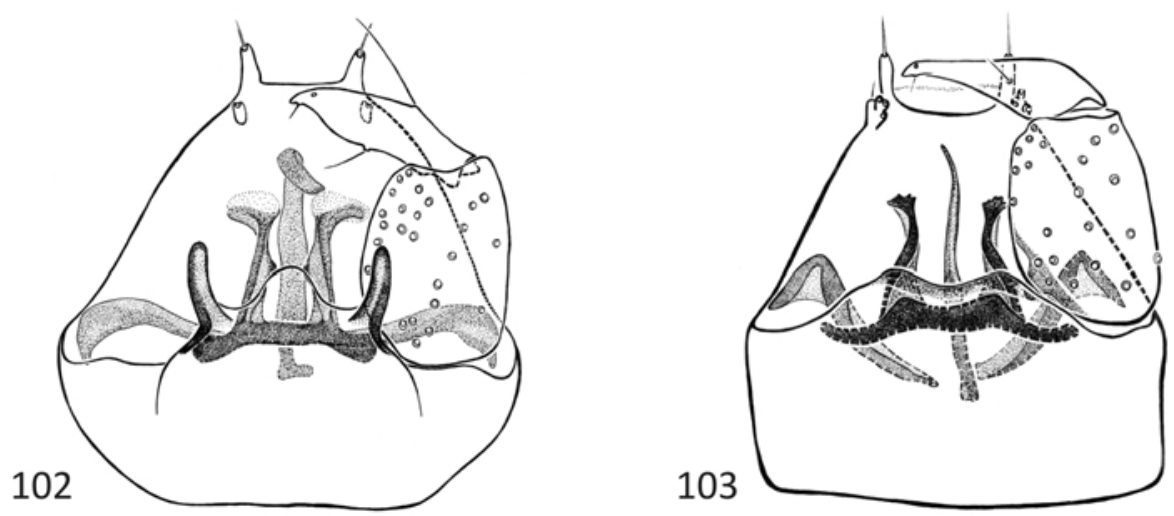

Figs 102-103. Male genitalia: 102 - Dasyhelea kyrenica Remm; 103 - D. similaris Remm. 


\section{Description}

Male. AR 0.81-0.87 $(\mathrm{n}=2)$. Clypeus entire, with lateral lobes and 6-7 long setae (Fig. 87). Palpal segment 3 slender, 56-68 $\mu \mathrm{m}$ long $(\mathrm{n}=3), \mathrm{PR}_{(\mathrm{III})} 3.50-4.86(\mathrm{n}=3)$. Transverse suture of thorax distinct (Fig. 79). Scutellum dark, with ca. 5 bristles. Wing length $0.84-$ $0.94 \mathrm{~mm}(\mathrm{n}=2)$, CR $0.40(\mathrm{n}=2)$. Legs dark, only tarsomeres somewhat paler. Genitalia (Figs 16, 102). Posterior margin of sternite 9 extended, rounded in apical part and bearing two submedian, finger-like projections. Gonocoxite short. Gonostylus swollen in the middle half, with a sharp pointed apex. Parameres nearly symmetrical, divided. Posterior process often bent apically. Aedeagus low-arched, with one pair of slightly twisted submedian projections.

Female. Subgenital plate with rectangular notum. Spermatheca spherical, with neck (REMM 1972).

Material examined $(n=2)$

Poland: Central Poland Lowlands. Czeszów near Wrocław, gravel pit, 30 August 2007, net, 1 male, leg. P.D. Central Western Carpathians. Chyżne near Nowy Targ, Wyżni Młyn on the Jeleśnia Stream, 29 July 2006, net, 1 male, leg. P.D.

Distribution and biology

Poland, Romania, Russia (Buryatia) (DOMINIAK \& SZADZIEWSKI 2010).

Dasyhelea kyrenica has been reported from two localities in southern Poland. Adult males were collected on a peat bog and in a gravel pit at the end of July and August respectively.

\section{Discussion}

Dasyhelea kyrenica could be confused with two other species described from South Primorye and Siberia, namely D. aithalodes REMM and D. hamardabani REMM. They differ slightly in the shapes of gonostylus, aedeagus and submedian processes of sternite 9 .

KNOZ \& BEUK (2002) mentioned D. kyrenica from the Netherlands, but this record is not provided with locality data and so is treated here as doubtful.

Only the paratypes of D. kyrenica have been found in the collection of the Tartu Ülikooli Loodusmuuseum.

\section{Dasyhelea similaris REMM, 1972}

(Fig. 103)

Dasyhelea similaris REMM, 1972: 77 (description and figure - male, female, Russia: Buryatia); REMM 1979: 50 (Estonia); Dominiak \& SzADZIEwski 2006: 6 (Poland); DominiaK \& SzAdziewski 2010: 24 (Romania).

\section{Diagnosis}

Clypeus entire. Male genitalia with sternite 9 slightly expanded, its apical part concave. Gonostylus broad basally and tapering to a point, wedge-shaped. Parameres nearly symmetrical. 


\section{Description}

Male. Clypeus entire, pentagonal, bearing 4-6 bristles. Palpal segment 3 cylindrical; length 51-57 $\mu \mathrm{m}(\mathrm{n}=2), \mathrm{PR}_{(\mathrm{III})}$ 3.64-4.07 $(\mathrm{n}=2)$. Transverse suture short but well visible. Scutellum dark. Wing length 0.76-0.78 mm $(n=2)$, CR 0.41-0.43 $(n=2)$. Genitalia (Fig. 103). Posterior margin of sternite 9 produced, with broad but shallow apical excavation. Gonocoxite short. Gonostylus wedge-shaped, swollen at the base and slender at the apex. Parameres nearly symmetrical, divided. Posterior process long and slender. Submedian projections of aedeagus long, slightly twisted, stout basally, with fringe-like tips.

Female. Margin of gonopore surrounded by strongly sclerotized plate. Spermatheca subspherical, with short neck, diameter $50 \mu \mathrm{m}$ (REMM 1972).

Material examined $(\mathbf{n}=\mathbf{1})$

Poland: EAstern BAltic LaKelands. Słupie near Suwałki, near the River Czarna Hańcza, peat bog, 24 May 2006, net, 1 male, leg. P.D.

\section{Distribution and biology}

Estonia, Poland, Romania, Russia (Buryatia) (DOMINIAK \& SZADZIEWSKI 2010).

In Poland D. similaris is known from the Wigry National Park. A single male was collected from a peat bog at the end of May.

\section{Discussion}

HAVELKA (1978) listed this species from mountains near Tübingen, but its presence is questioned in the Checkliste der Dipteren Deutschlands (HAVElKA \& AGUILAR 1999). Because the record from Germany has not been confirmed, it is regarded here as doubtful.

The holotype and all paratypes of $D$. similaris were deposited by Remm in the Tartu Ülikooli Loodusmuuseum in Estonia. Only one female has been found in this collection.

\section{Dasyhelea arenivaga MACFIE, 1943}

(Figs 85, 89, 104)

Dasyhelea inconspicuosa var. arenivaga MACFIE, 1943: 151 (description, in key - male, female, Egypt). Dasyhelea arenivaga: SZADZIEWSKI 1986: 55 (description and figure - male, Algeria, Poland);

DELÉCOLle 1998 (1999): 137 (Spain); TÓthOvÁ et al. 2004a: 309 (Czech Republic); DominiAK \& SzADZIEWSKi 2006: 6 (Poland); DominiaK et al. 2007a: 260 (figure - male, Israel); DominiaK \& SZADZIEWSKi 2010: 5 (= flavoscutellata sensu SzADZIEWSKI 1983, comments on biology, Bulgaria, Romania, Ukraine); SZADZIEWSKI et al. 2011: 641 (United Arab Emirates).

Dasyhelea flavoscutellata: SzADZIEWSKI 1983: 66 (saline habitats, Poland); SzADZIEWSKI 1998: 103 (Switzerland). Nec D. flavoscutellata (ZETTERSTEDT, 1850).

\section{Diagnosis}

Apicolateral processes of tergite 9 in male genitalia cylindrical, slightly expanded apically, with single setae located near apex. Sternite 9 with rounded apex, joined to aedeagus by a conspicuous bridge. 

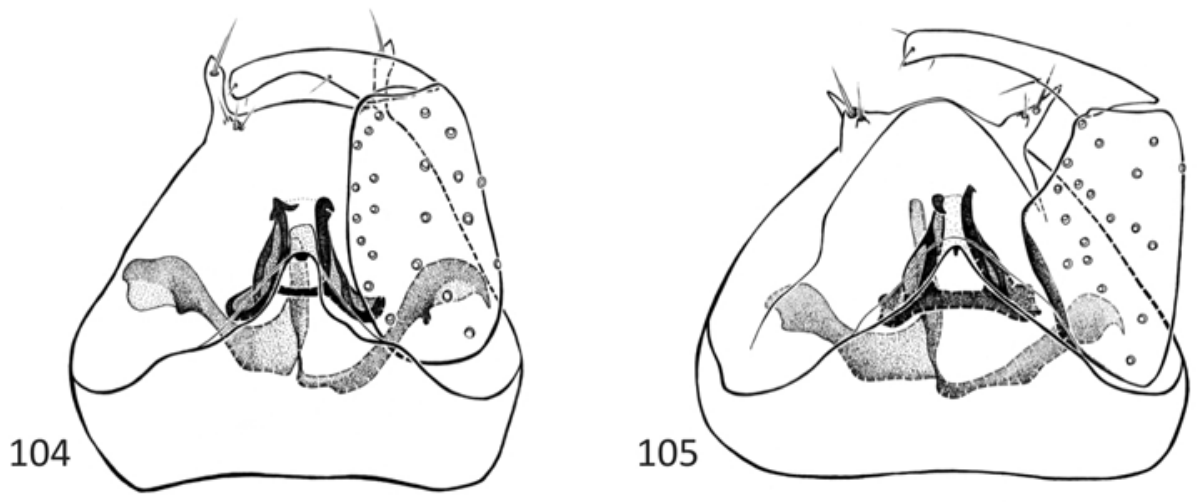

Figs 104-105. Male genitalia: 104 - Dasyhelea arenivaga MACFIE; 105 - D. flavoscutellata (ZETTERSTEDT).

\section{Description}

Male. Antennal segments 10-12 with two rows of long setae (Fig. 85), AR 0.92-1.05 $(\mathrm{n}=12)$. Palpal segment 3 elongate (Fig. 89); length 52-73 $\mu \mathrm{m}(\mathrm{n}=12), \mathrm{PR}_{\text {(III) }} 4.14-5.62$ $(\mathrm{n}=12)$. Transverse suture absent. Scutellum yellow or dark, bearing 8-9 bristles and a few smaller setae. Wing length $0.80-0.97 \mathrm{~mm}(\mathrm{n}=12)$, CR 0.43-0.46 $(\mathrm{n}=12)$. Legs dark, tarsi somewhat paler. Genitalia (Fig. 104). Apicolateral processes of tergite 9 cylindrical, slightly swollen and with small triangular extension in apical half. Setae located near apex. Posterior margin of sternite 9 extended, with rounded apex, fused to aedeagus by conspicuous bridge. Gonostylus arched, with slightly enlarged apex. Parameres asymmetrical, fused. Posterior process rather short, broadly fused to left basal arm. Aedeagus with a pair of parallel apicolateral projections.

Female. Spermatheca well-sclerotized, spherical, diameter $41 \mu$ m (MACFIE 1943).

Material examined $(n=53)$

Poland: Southern Baltic Coastlands. Brzyno near Żarnowiec, Apiaceae, 2 June 1982, 1 male, leg. R.Sz. Gdańsk, Sobieszewska Is., Górki Wschodnie, estuary, 18 August 1977, 1 male, leg. R.Sz. Karsibór on Uznam Is., saline habitat, 9 June 1975, 1 male, leg. R.Sz. Mieroszyno near Władysławowo, Bielawskie Błota, near bog, Pinus sp., 29 June 1979, 1 male, leg. R.Sz. SoutherN BAltic LAKelands. Aleksandrów Kujawski, saline habitat, soil, 16 April - 30 May 1975, ex cult., 7 males, leg. R.Sz. Inowrocław Mątwy, saline habitat, 15 May 1975, 6 males, leg. R.Sz.; 25 April 2008, ex cult., 11-13 May 2008, 3 males, leg. P.D.; Salicornietum, 5 March - 15 April 1975, ex cult., 3 males, leg. R.Sz.; soil, 5 March 1975, ex cult., 6 males, leg. R.Sz.; 5 March - 15 April 1975, ex cult., 1 male, leg. R.Sz.; Festucetum, soil, 18 April 1975, ex cult., 2 males, leg. R.Sz. Inowrocław Mątwy, Apiaceae, 19 August 2009, net, 1 male, leg. P.D. Inowrocław Rąbin, 4 August 1973, 1 male, leg. R.Sz. Janikowo near Inowrocław, saline habitat, 14 May 1975, 1 male, leg. R.Sz.; 5 May 1975, 
1 male, leg. R.Sz.; 4 August 1975, 1 male, leg. R.Sz. EASTERn BALtic LaKELANDS. Słupie near Suwałki, near the River Czarna Hańcza, peat bog, 24 May 2006, net, 1 male, leg. P.D. Wiatrołuża Pierwsza near Suwałki, riparian forest on the River Wiatrołuża, 2 July 2005, net, 2 males, leg. P.D. Poszeszupie near Suwałki, Chaerophyllum sp., 3 July 1993, 2 males, leg. J.K. SUdETy MTs. Radomierz near Jelenia Góra, Trzcińskie Mokradła, 25 June 1993, 1 male, leg. E.K. CENTRAL Western Carpathians. Dolina Małej Łąki in the Tatra Mts., 11 July 2003, net, 1 male, leg. M.G.K. Czarny Dunajec near Nowy Targ, Baligówka peat bog, 28 June 2006, net, 2 males, leg. P.D. Szafranówka in the Pieniny Mts., 24 June 2003, net, 5 males, leg. M.G.K. EASTERN BESKID MTS. Muczne near Ustrzyki Górne, Tarnawa Reserve, peat bog, 13 July 2005, net, 2 males, leg. P.D.

\section{Distribution and biology}

Poland, Czech Republic, Switzerland, Romania, Ukraine (Crimea), Bulgaria, Spain, Algeria, Egypt, Israel, United Arab Emirates (DomINIAK \& SzADZIEWSKI 2010, SZADZIEWSKI et al. 2011).

This species is known from several localities in Poland. Adult males of D. arenivaga were frequently collected in peat bogs and meadows. Larvae have been found in inland saline habitats. Adults of $D$. arenivaga fly from May to August. This species is known to visit umbelliferous and tamarisk flowers (DOMINIAK \& SZADZIEWSKI 2010).

The syntype male of $D$. inconspicuosa var. arenivaga MACFIE is deposited in the collection of the Natural History Museum, London (SZADZIEWSKI 1986).

\section{Dasyhelea flavoscutellata (ZETTERSTEDT, 1850)}

(Figs 82, 105)

Ceratopogon flavoscutellatus ZETTERSTEDT, 1850: 3648 (description - female, Norway).

Dasyhelea flavoscutellata: REMM 1962: 120 (description - male, female, figure - male, breeding site

- littoral zone of lakes, puddles, Estonia); REMM 1967: 16 (Azerbaijan, Georgia); REMM

\& ZHogolev 1968: 832 (Ukraine: Crimea); REMM 1973a: 172 (Mongolia); ReMM 1973b: 355

(Hungary); HAVELKA 1976a: 63 (Germany); HAVELKA 1976b: 225 (description and figure

- male, female, syn.: = egens, = flavoscapula, $=$ alonensis, $=$ heraclae, $=$ halobia $)$; HAVELKA 1978: 177 (syn.: = egens, = alonensis, = heraclae, = halobia, Germany); REMM 1979: 51 (Estonia); SzAdZIEwSKi 1986: 51 (description and figure - male, female, Poland); DelÉColle et al. 1991: 256 (France); KNOZ et al. 2004: 84 (Czech Republic); TóthOVÁ et al. 2004a: 309 (Czech Republic); TóthovÁ et al. 2004b: 146 (Czech Republic); TóthovÁ \& KNOZ 2006: 106 (Czech Republic); DOMINIAK \& SZADZIEWSKI 2010: 15 (comments on synonymy and distribution, Algeria); GwizDALSKA-KenTZER 2011: 37 (Poland).

\section{Diagnosis}

Apicolateral processes of tergite 9 triangular, tapering sharply to a point, with seta located in the middle of the process. Posterior margin of sternite 9 with rounded apex, joined to aedeagus by a conspicuous bridge.

\section{Description}

Male. AR 0.92-1.02 $(\mathrm{n}=2)$. Frontal sclerite elliptical (Fig. 82). Palpal segment 3 long 
and slender; length 61-76 $\mu \mathrm{m}(\mathrm{n}=4), \mathrm{PR}_{\text {(III) }} 3.95-5.00(\mathrm{n}=3)$. Transverse suture absent. Scutellum yellow or dark, with 7 bristles and some additional setae. Wing length 0.83-1.11 $\mathrm{mm}(\mathrm{n}=4)$, CR 0.42-0.46 ( $\mathrm{n}=4)$. Legs dark, knees black, tarsi pale. Genitalia (Fig. 105). Apicolateral processes of tergite 9 elongate, triangular and sharp. Strong single seta located in the middle of the process. Distal margin of sternite 9 elongate, with rounded apex. Bridge joining sternite 9 and aedeagus conspicuous. Gonostylus, parameres and apicolateral projections of aedeagus similar to those in the former species.

Female. Notum of subgenital plate with semicircular opening. Spermatheca with neck, well-sclerotized, length $41 \mu \mathrm{m}$ (SZADZIEWSKI 1986).

Material examined $(\mathbf{n}=\mathbf{5})$

Poland: Southern BaLtic LaKelands. Gdynia Bernadowo, Łęg nad Swelinią Reserve, 9 August 2011, net, 1 male, leg. M.G.K. EASTERn BALTiC LAKELANDS. Silec near Kętrzyn, forest, May/2.1980, 1 male, leg. R.Sz.; on window in house, May/2.1980, 1 male, leg. R.Sz. OUTER WeSTERN Carpathians. Moszczenica near Żywiec, 15 July 1992, 1 male, leg. J.K. Central Western Carpathians. Sokolica in the Pieniny Mts., 28 June 2003, net, 1 male, leg. M.G.K.

Distribution and biology

Norway, Estonia, Germany, Poland, Czech Republic, France, Hungary, Ukraine (Crimea), Georgia, Azerbaijan, Mongolia, Algeria (DOMINIAK \& SZADZIEWSKI 2010).

Polish records of this species are rather rare. Male specimens of $D$. flavoscutellata were collected in spring and summer from two localities in the lakelands of northern Poland and two others in the Carpathian Mountains.

The pupae of $D$. flavoscutellata inhabit shallow water bodies. They were found among plants in the littoral zone of lakes and in puddles on forest roads (REMM 1962).

\section{Discussion}

Most of the old records of $D$. flavoscutellata are doubtful. This species has also been listed from Denmark (PeTERSEN \& ACHIM 2001) and the Azores (DelÉCOlLe 2002), but no locality data has been given.

The holotype of $D$. flavoscutellata is deposited in the collection of the Museum of Zoology of Lund University, Sweden (SZADZIEWSKI 1986).

\section{subgenus Sebessia}

Sebessia Remm, 1979: 55. Type species Dasyhelea flavopyga ZiLAHI-Sebess, 1940: 49 (by original designation).

\section{Diagnosis}

Body dark, velvet black or metallic pruinose. Frontal sclerite narrow, elliptical, very often with apicolateral borders and lateral lobate projections. Apex of last antennal segment conical, without apical extension. Clypeus usually elongate, composed of two sclerites. Wing with two radial cells. 


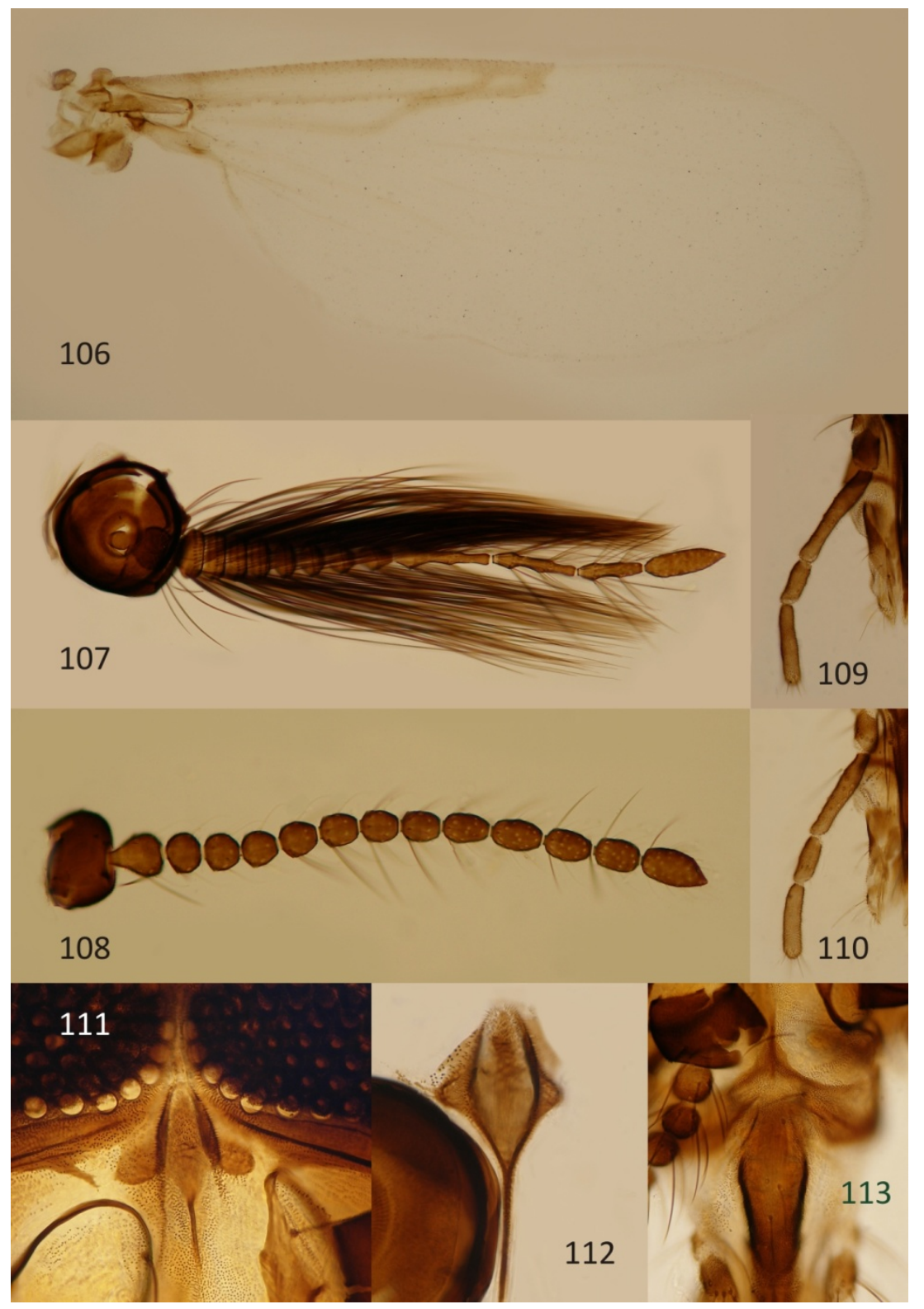

Figs 106-113. Dasyhelea holosericea (MEIGEN), male (107, 108, 112), female (106, 109, 110, 111, 113).

106 - wing, 107, 108 - antenna, 109, 110 - palpus, 111, 112 - frontal sclerite, 113 - clypeus. 
Male. Posterior margin of tergite 9 rounded, with short apicolateral processes. Posterior margin of sternite 9 with an envelope covering whole aedeagus. Parameres totally or nearly symmetrical, divided or fused.

Female. Subgenital plate simple, somewhat arch-shaped with irregular, semicircular or rectangular anterior margin, or sometimes bipartite, with separate anteromedian sclerite. Two functional spermathecae and one rudimentary present.

\section{Discussion}

The species of the cincta group (WIRTH 1952) and the holosericea group (REMM 1979) belong to Sebessia. Dasyhelea species of the recently proposed patagonica group (DíAz et al. 2010) present a similar combination of morphological characters and should probably be also included in this subgenus.

All known pupae of Sebessia species have quite long, tubular and annulated respiratory horns, with both apical and lateral spiracles.

This subgenus is represented by four species in the European fauna, namely D. acuminata KiefFer, D. flavopyga ZilahI-Sebess, D. holosericea (MeIgen), D. ledi REMM. So far, only one of them is known to occur in Poland.

\section{Dasyhelea holosericea (MEIGEN, 1804)}

(Figs 106, 107, 108, 109, 110, 111, 112, 113, 114, 115, 116)

Ceratopogon holosericeus MEIGEN, 1804: 27 (description - male, female, Europe); GoETGHEBUER 1922: 52 (description and figure - male, female, Meigen's collection).

Dasyhelea holosericea: GoETGHEBUER 1922: 58 (genus Dasyhelea); KIEFFER 1925c: 69 (description, in key - male, female, figure - female); GOETGHEBUER 1934: 33 (description, in key - male, female, figure - female); STOR̊ 1939: 18 (Finland); KARL 1940: 30 (Poland); KRIVOSHEINA 1957: 419 (Russia: Ryazan Oblast); REMM 1962: 125 (description, figure - male, Estonia); REMM 1966: 60 (Lithuania); REMM 1979: 49 (Estonia); DelÉCOLLE et al. 1991: 256 (France); DominIAK \& SZADZIEWSKI 2010: 17 (distribution).

\section{Diagnosis}

Posterior margin of sternite 9 in male genitalia extended, with a pair of horn-like appendages. Posterior process slender, slightly swollen in apical part. Upper half of notum in female subgenital plate broad basally and narrow apically.

\section{Description}

Male. Antennal segments 2-5 appressed, 10-12 with two rows of long setae (Fig. 107), AR 1.04-1.24 $(\mathrm{n}=5)$. Frontal sclerite elliptical, with lateral lobes (Fig. 102). Clypeus elongate, bearing 4-9 setae. Palpal segment 3 slender (Fig. 108), 109-134 $\mu \mathrm{m}$ long $(\mathrm{n}=5)$, $\mathrm{PR}_{\text {(III) }}$ 4.64-6.09 $(\mathrm{n}=5)$. Fifth palpal segment 1.5 times longer than the fourth one. Scutellum dark, with 10-12 bristles and 3-5 smaller setae. Wing with two radial cells, cell $\mathrm{r}_{1}$ slit like. Wing length 1.23-1.36 mm $(\mathrm{n}=5)$, CR 0.48-0.52 $(\mathrm{n}=5)$. Legs dark, only 
tarsomeres somewhat paler. Genitalia (Fig. 114). Posterior margin of tergite 9 rounded, with short and conical apicolateral processes. Apex of sternite 9 with prominent, heavily sclerotized posterior horn-like processes. Gonostylus short, stout and sharply pointed. Parameres divided, symmetrical. Posterior process long and slender, with spoon-shaped apex. Aedeagus entirely covered by sternite 9 . Median projections of aedeagus in the form of short cones.

Female. AR 0.80-0.91 $(\mathrm{n}=3)$. Frontal sclerite elliptical, with lobate lateral processes (Fig. 111). Clypeus elongate, with 6 setae (Fig. 113). Third palpal segment slender (Fig. 110), 83-100 $\mu \mathrm{m}$ long $(\mathrm{n}=3), \mathrm{PR}_{\text {(III) }} 4.61-4.76(\mathrm{n}=3)$. Fifth palpal segment as in male. Scutellum dark, with 12 bristles and 6-8 smaller setae. Two radial cell present, cell $\mathrm{r}_{1}$ slitlike (Fig. 106). Wing length 1.10-1.19 mm $(\mathrm{n}=3)$, CR 0.49-0.52 $(\mathrm{n}=3)$. Legs dark, tarsi somewhat paler. Genitalia (Figs 115, 116). Notum in subgenital plate weakly sclerotized mesally. Its anterior part slightly concave. Upper half of notum broad at base and distinctly tapering to a point. Two unequal, functional spermathecae present. Both with short neck, ovoid, dimension 64-68 $\mu \mathrm{m}(\mathrm{n}=3) \times 39-44 \mu \mathrm{m}(\mathrm{n}=3)$ and 71-75 $\mu \mathrm{m}(\mathrm{n}=3) \times 40-53 \mu \mathrm{m}$ $(\mathrm{n}=3)$. Third spermatheca rudimentary, 14-29 $\mu \mathrm{m}(\mathrm{n}=2)$ long and 5-7 $\mu \mathrm{m}$ wide $(\mathrm{n}=3)$.

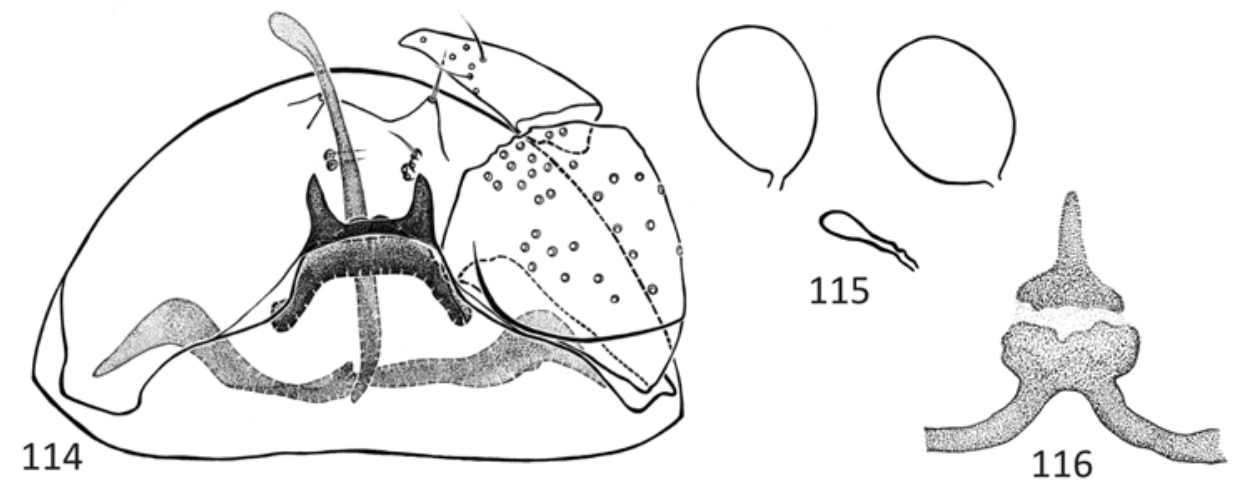

Figs 114-116. Dasyhelea holosericea (MEIGEN): 114 - male genitalia, 115 - seminal capsules, 116 female subgenital plate.

Material examined $(\mathbf{n}=\mathbf{5})$

Poland: Southern Baltic Coastlands. Stolp i. P., Veddin [Widzino near Słupsk], 15 May 1912, 2 males, leg. O. Karl, Muz. Zool. Polonicum Warszawa, coll. O. Karl, 29/47, [MIIZ].

Other specimens

France: Dasyhelea holosericea, Auvergne, Lavours, 1.7.86, VIII, P2, 15, B86, Musée Zoologique Strasbourg, 1 female, [MZSF]. Dasyhelea holosericea, Auvergne, Lavours, 18.7.86, VIII, P1, 7, B86, Musée Zoologique Strasbourg, 1 female, [MZSF]. Dasyhelea holosericea, Auvergne, Lavours, 18.7.86, VIII, P1, 7, B86, Musée Zoologique Strasbourg, 1 female, [MZSF]. 


\section{Distribution and biology}

Finland, Estonia, Lithuania, Poland, Russia (Ryazan Oblast), France (DOMINIAK \& SZADZIEWSKI 2010).

The only report from Poland (KARL 1940) was based on male specimens collected in May 1912 near Stupsk. In spite of intensive searches in different parts of the country, $D$. holosericea has not been found again. In Europe adults were recorded from May to September (STORÅ 1939, KRIVOSHEINA 1957, REMM 1962, 1966).

\section{Discussion}

The Yakutian species D. velutina REMM is very similar to D. holosericea. According to the original description, $D$. velutina has slightly infuscated wings but there are hardly any differences in the structure of the male genitalia and in the shape of the female subgenital plate. These two names should probably be synonymized.

The records from Germany, Great Britain (KIEFFER 1925c) and Austria (GOETGHEBUER 1934), as well as the first report from Poland (MAYER 1934b), are doubtful.

\section{Concluding comments}

Up to now 30 species of Dasyhelea are known to occur in Poland, representing five subgenera: Dasyhelea s. str. (6 spp.), Dicryptoscena (6 spp.), Prokempia (3 spp.), Pseudoculicoides (14 spp.) and Sebessia (1 sp.). Among the Polish biting midge fauna, most species of the genus Dasyhelea are primarily arboreal, widely distributed Palaearctic species. The most species-rich samples were collected in the following regions of Poland: Eastern Baltic Lakelands (19 spp.), Southern Baltic Lakelands (16 spp.), Southern Baltic Coastlands (14 spp.) and Central Western Carpathians (12 spp.). These results can be explained by the high intensity of research focused on these regions. The most frequently reported species in this country are $D$. flavifrons (listed from 11 physiographic regions), D. pallidiventris (from 8 regions), and six other species from six regions: D. arenivaga, D. bilobata, D. calycata, D. flaviventris, D. modesta and D. notata. Many species of Dasyhelea are known from only single localities, so species like D. auli, D. furva, D. holosericea, D. similaris, D. stackelbergi, D. turanicola and D. unguistyla are considered rare.

The larvae of Dasyhelea were found in sap and tree holes (D. flavifrons), water accumulated in gutters and sinks ( $D$. bilineata), in peat bogs (D. bilobata, D. turficola), moist soil near fresh- (D. flaviventris, D. pallidiventris) and salt-water bodies (D. arenivaga, D. calycata, D. leptoclada, D. modesta, D. pallidiventris, D. turficola, D. unguistyla). According to SzADZIEWSKI (1983), the last group contains known halobiontic (D. leptoclada, D. unguistyla), halophilic (D. calycata) and haloxenic species (D. arenivaga, D. modesta, D. pallidiventris, D. turficola). It is very probable that D. bilineata, D. stellata and D. bicrenata can also reproduce in brackish water, because 
adults of these species were often collected near the seashore. Furthermore, Dasyhelea species are quite common inhabitants of wetlands, and more than half of the Polish species are known to inhabit peat bogs. These are: D. arenivaga, D. bilobata, D. corinneae, D. dampfi, D. flavifrons, $D$. flaviventris, $D$. kyrenica, D. malleola, D. modesta, D. notata, D. pallidiventris, $D$. similaris, $D$. stackelbergi, $D$. thienemanni, $D$. turficola, and very likely $D$. holosericea as well. Adults are known to visit flowers for nectar, and 16 species were found on umbelliferous flowers: D. arenivaga, D. auli, D. bicrenata, D. bifida, D. bilobata, D. calycata, D. flavifrons, D. flaviventris, D. furva, D. leptoclada, D. lucida, D. modesta, D. notata, D. pallidiventris, D. thienemanni and D. turficola. Outside Poland, Dasyhelea were also collected from tamarisk (D. arenivaga, D. leptoclada, D. turanicola) and euphorbia (D. lucida) (Remm 1967, Remm \& NAZARMukHamedov 1969, DominiaK \& SZADZIEWSKI 2010).

\section{REFERENCES}

Ashe P., O'Connor J.P., ChAndler P.J. 2007. Dasyhelea (Dasyhelea) saxicola (EDWARdS) (Diptera: Ceratopogonidae) new to Ireland and Wales from limestone rock pools with a review of its ecology and distribution. Entomologist's Monthly Magazine 143: 113-126.

BeCKenBACH A.T., BoRKENT A. 2003. Molecular analysis of the biting midges (Diptera: Ceratopogonidae), based on mitochondrial cytochrome oxidase subunit 2. Molecular Phylogenetics and Evolution 27: 21-35.

BERNOTIENEE R. 2002. New data on the fauna of biting midges (Diptera, Ceratopogonidae) from Lithuania. Acta Zoologica Lituanica 12: 288-293.

Boorman J., Coluzzi M., Contini C., Ferrarese U., Rivosecchi L., Rossaro B., Sabatini A., Wagner R. 1995. Diptera Culicomorpha. [In:] A. Minelli, S. Ruffo, S. La Posta (eds). Checklist delle Specie della fauna Italiana. Calderini, RCS, Milano 65: 1-32.

Boorman J., van HARTEN A. 2002. Some Ceratopogonidae (Insecta: Diptera) from the Arabian Peninsula, with particular reference to the Republic of Yemen. Fauna of Arabia 19: 427-462.

Borkent A. 1997. New species of biting midges from Hawaii (Diptera: Ceratopogonidae). Memoirs of the Entomological Society of Washington 18: 84-94.

BORKENT A. 2012. World species of biting midges (Diptera: Ceratopogonidae). Available at: http://www.inhs.uiuc.edu/cee/FLYTREE/CeratopogonidaeCatalog.pdf. (Last updated: February 28, 2012).

BORKENT A., Forster L. 1986. Review of the Dasyhelea fasciigera species group (Diptera: Ceratopogonidae) with a revision of the Nearctic species. Canadian Journal of Zoology 64: 12801287.

BORKENT A., WiRTH W.W. 1997. World species of biting midges (Diptera: Ceratopogonidae). Bulletin of the American Museum of Natural History 233: 1-257.

BRODSKAYA N.K. 1995. A new species of biting midge of the genus Dasyhelea KIEFF. (Diptera, Ceratopogonidae) from Leningrad Region. International Journal of Dipterological Research 6: 9-12.

BrodSKAYA N.K. 1996. A new species of biting midge of the genus Dasyhelea KIEFFER (Diptera, Ceratopogonidae) from Kirghistan. International Journal of Dipterological Research 7: 193-196.

CANTREll M.A., MCLaChlan A.J. 1982. Habitat duration and dipteran larvae in tropical rain pools. Oikos 38: 343-348. 
CARTER H.F., Ingram A., MACFIE J.W.S. 1921. Observations on the ceratopogonine midges of the Gold Coast, with descriptions of new species. IV. Annals of Tropical Medicine and Parasitology 15: $177-212$.

CHANDLER P.J. (ed.) 1998. Checklists of insects of the British Isles (New Series). Part 1: Diptera (Incorporating a List of Irish Diptera). Handbooks for the identification of British insects 12. Royal Entomological Society, London, $234 \mathrm{pp}$.

Chandler P.J., Ashe P., O'ConNor J.P. 2008. Notes on Ceratopogonidae (Diptera) including some new national records for Britain and Ireland. Dipterists Digest 15: 73-94.

Clastrier J. 1966. Cératopogonidés des iles Canaries (Dipt. Nematocera). Annales de la Société Entomologique de France, N.S. 2: 693-710.

Clastrier J., RiouX J.A., Descous S. 1961. Notes sur les Cératopogonidés. XII. Cératopogonidés de Nord-Tchad. Archives de l'Institut Pasteur Algérie 39: 49-98.

Coquillett D.W. 1901. New Diptera in the U.S. National Museum. Proceedings of the United States National Museum 23: 593-618.

DAMIAN-GEORGESCU A. 1973. Contribuții la cunoaşterea genurilor Forcipomyia şi Dasyhelea (Diptera: Ceratopogonidae) din România. Studii şi Cercetari de Biologie, Seria Zoologie 25: 447-454.

Damian-Georgescu A. 1975. Ceratopogonidae (Diptera) noi pentru fauna României. Studii şi Cercetari de Biologie, Seria Zoologie 27: 97-100.

DE MeIJERE J.C.H. 1946. Zevende Supplement op de Nieuwe Naamlijst van Nederlandsche Diptera van 1898. (Eerste Supplement op mijne Naamlijst van 1939). Tijdschrift voor Entomologie 87: 1-25.

DelÉCOLLE J.-C. 1998 (1999). Ceratopogonidés (Diptera, Nematocera) do Los Monegros. Boletin de la Sociedad Entomologica Aragonesa 24: 137.

DeléColle J.-C. 2002. Ceratopogonidae. [In:] M. CARles-TolrÁ, Huorth-Andersen (eds). Catálogo de los Diptera de España, Portugal y Andora (Insecta). Monografías de la Sociedad Entomológica Aragonesa Zaragoza 8: 26-33.

DelÉCOlLE J.-C., RieB J.-P. 1990. Liste faunistique complémentaire des Cératopogonidés d'Alsace (Dipt. Nematocera). Bulletin de la Société Entomologique de France 95: 183-185.

DeléColle J.-C., RIEB J.-P. 1993. Contribution à l'étude des Cératopogonidés d'Espagne. Description de Forcipomyia (Thyridomyia) blascovi n. sp. (Diptera, Nematocera). Nouvelle Revue d'Entomologie (N.S) 10: 109-120.

DelÉCOLle J.-C., RieB J.-P., BRUnhes J. 1991. Note préliminaire sur les Diptères Cératopogonidés de la tourbière eutrophe du marais de Lavours (Vallée du Rhône, Ain). Bulletin de la Société Entomologique de France 96: 255-256.

DíAz F., Spinelli G.R., Ronderos M.M. 2010. A revision of the species allied to Dasyhelea patagonica INGRAM and MACFIE (Diptera: Ceratopogonidae). Journal of Natural History 44: 2825-2849.

DisNeY R.H.L. 1975. A midge (Diptera, Ceratopogonidae) new to Britain that is abundant in the limestone pavement of the Yorkshire Pennines. Entomologist's Monthly Magazine 110: 227-228.

Disney R.H.L., WiRTH W.W. 1982. A midge (Dipt., Ceratopogonidae) new to Britain from teasel axil-waters in Suffolk. Entomologist's Monthly Magazine 118: 233-234.

DoDSON S.I. 1987. Animal assemblages in temporary desert rock pools: aspects of the ecology of Dasyhelea sublettei (Diptera: Ceratopogonidae). Journal of the North American Benthological Society 6: 65-71.

DominiaK P. 2005. Biting midges (Diptera: Ceratopogonidae) developing in hollows and sap of various species of trees. Dipteron 21: 5-6. [in Polish, English abstract].

DominiaK P. 2009. Biting midges (Diptera: Ceratopogonidae). [In:] J. Herbich, M. Ciechanowski (eds). The nature of Kurze Grzędy and Staniszewskie Błoto reserves in the Kashubian Lakeland. Fundacja Rozwoju Uniwersytetu Gdańskiego, Gdańsk, pp.: 199-205. [in Polish]. 
Dominiak P., Braverman Y., SzAdZiEwSki R. 2007a. New records of biting midges of the genus Dasyhelea from Israel, with notes on synonymy in the genera Culicoides and Forcipomyia (Diptera: Ceratopogonidae). Polish Journal of Entomology 76: 257-265.

DominiaK P., GWIZDALSKA-KENTZER M., SZADZIEWSKI R. 2007b. New faunistic records of the biting midges (Diptera: Ceratopogonidae) from the Bieszczady National Park. Dipteron 23: 2-6. [in Polish, English abstract].

DominiaK P., MichalcZUK W. 2009. Two species of biting midges (Diptera: Ceratopogonidae) new to the Polish fauna. Dipteron 25: 8-13. [in Polish, English abstract].

DominiaK P., SzADZIEWSKi R. 2006. Biting midges of the genus Dasyhelea Kieffer, 1911 (Diptera: Ceratopogonidae) collected from Polish peat-bogs. Dipteron 22: 4-7. [in Polish, English abstract].

DominiaK P., SzADZIEWSKi R. 2008. New synonymy and reports of biting midges in Poland (Diptera: Ceratopogonidae). Polish Journal of Entomology 77: 145-150.

DominiaK P., SzADZIEWSKi R. 2010. Distribution and new synonymy in European biting midges of the genus Dasyhelea Kieffer (Diptera: Ceratopogonidae). Zootaxa 2437: 1-37.

EDWARDS F.W. 1926. On the British biting midges. Transactions of the Royal Entomological Society of London 74: 389-426.

EDWARDS F.W. 1929. British non-biting midges (Diptera, Chironomidae). Transactions of the Royal Entomological Society of London 77: 279-430.

EdWARDS F.W. 1933. Some Perthshire Diptera. Scottish Naturalist 201: 87-92.

Enderlein G. 1936. Ordnung: Zweiflügler, Diptera. Abt. 16. [In:] P. Brohmer, P. Ehrmann, G. Ulmer (eds). Die Tierwelt Mitteleuropas 6: Insekten. III Teil. Leipzig, 259 pp.

Evenhuis N. 2012. Abbreviations for Insect and Spider Collections of the World. Available at: http://hbs.bishopmuseum.org/codens/codens-inst.html (Last updated: May 2, 2012).

Forattini O.P., RABello E.X. 1957. Dados sobre Dasyhelea KiefFer, 1911 do Brasil (Diptera, Ceratopogonidae). Revista Brasileira de Entomologia 7: 241-250.

FÜRST VON LIEVEN A. 1998. Functional morphology and phylogeny of the larval feeding apparatus in the Dasyheleinae and Forcipomyiinae (Diptera, Ceratopogonidae). Deutsche Entomologische Zeitschrift 45: 49-64.

García SÁnchez M.R., Sahuquillo Herráiz C. 1986. Descripción de dos Ceratopogónidos (Diptera, Nematocera) nuevos para la ciencia. Eos 62: 95-101.

Gil Collado J., Sahuquillo Herráiz C. 1983. Claves de identificación de Ceratopogonidae de España Peninsular. I. Subfamilias Forcipomyiinae y Dasyheleinae (Dipt., Nematocera). Graellsia 39: 31-45.

GLUKHOVA V.M. 1981. A comparative morphological review of the mouth parts of the females and the males in the subfamilies Dasyheleinae and Forcipomyiinae (Diptera, Ceratopogonidae). Entomologičeskoe Obozrenie 60: 62-76. [in Russian, English title].

Glukhova V.M., BrodSKAYA N.K. 1997. Description of a new species of non-blood-sucking biting midge of the genus Dasyhelea KIEFFER (Diptera, Ceratopogonidae) from Karelia. Entomologičeskoe Obozrenie 76: 443-447 [in Russian, English abstract].

Goetghebuer D.M. 1910. Description de diptères chironomides nouveaux. Revue Mensuelle de la Société Entomologique Namuroise 10: 96-97.

Goetghebuer D.M. 1914. Contribution a l'étude des Chironomides de Belgique. Annales de Biologie Lacustre 7: 165-229.

GoetgheBuer D.M. 1919. Observations sur les larves et nymphes de quelques Chironomides de Belgique. Annales de Biologie Lacustre 9: 51-78.

Goetghebuer D.M. 1920. Ceratopogoninae de Belgique. Mémoires du Musée Royal d'Histoire Naturelle de Belgique 8: 2-116. 
Goetghebuer D.M. 1921. Chironomides de Belgique et spécialement de la zone des Flandres. Mémoires du Musée Royal d'Histoire Naturelle de Belgique 8: 1-208.

Goetghebuer D.M. 1922. Étude critique des Ceratopogon de la collection MeigEN conservée au Museum National d'Historie naturelle de Paris. Extrait du Bulletin de la Société Entomologique de Belgique 4: 50-59.

GoetgheBuer D.M. 1925. Notes biologiques et morphologiques sur Dasyhelea bilineata Goetgh. [In:] P. LECHEVALIER (ed.). Encyclopedie Entomologique, Diptera, recueil d'études biologiques et systematiques sur les Diptères du Globe, Paris, 1924-1953, pp.: 121-124.

Goetghebuer D.M. 1927. Nouveaux matériaux pour l'étude de la Faune des Chironomides de Belgique. Annales de Biologie Lacustre 15: 93-104.

Goetghebuer D.M. 1931. Ceratopogonidae et Chironomidae nouveaux d'Europe. Bulletin et Annales de la Société Entomologique de Belgique 71: 211-218.

GoEtGheBuer D.M. 1932. Ceratopogonidae et Chironomidae nouveaux ou peu connus d'Europe. Bulletin et Annales de la Société Entomologique de Belgique 72: 125-130.

Goetghebuer D.M. 1933a. Catalogue des Cératopogonides de Belgique. Bulletin et Annales de la Société Entomologique de Belgique 73: 363-372.

Goetghebuer D.M. 1933b. Ceratopogonidae et Chironomidae du Congo Belge. Revue de Zoologie et de Botanique Africaines 24: 129-151.

GoetgheBuer D.M. 1934. A. Die Imagines. Heleidae (Ceratopogonidae). 13a. [In:] E. LindNER (ed.). Die Fliegen der palaearktischen Region. E. Schweizerbart'sche Verlagsbuchhandlung, Stuttgart, pp.: 1-94.

Goetghebuer D.M., Lindroth C.H. 1931. Fam. Ceratopogonidae und Chironomidae. [In:] Die Insektenfauna Islands. Zoologiska Bidrag från Uppsala 13: 274-285.

Goetghebuer D.M., TIMON DAVID J. 1937. Contribution a l'étude des diptères halophiles et halobies du littoral méditerranéen Chironomides et Cératopogonides de l'îlot de Planier. Bulletin et Annales de la Société Royale Entomologique de Belgique 77: 409-416.

Gosseries J. 1991. Ceratopogonidae. [In:] P. Grootaert, L. De Bruyn, M. De Meyer (eds). Catalogue of the Diptera of Belgium. Institut Royal des Sciences Naturelles de Belgique Documents de Travail 70: 41-45.

Graves R.C., GRaves A.C.F. 1985. Diptera associated with shelf fungi and certain other microhabitats in the highlands area of western North Carolina. Entomological News 96: 87-92.

GRogan W.L., WiENERS J.A. 2006. A new species of the biting midge genus Dasyhelea KiefFER (Diptera: Ceratopogonidae) from the Bahamas. Proceedings of the Entomological Society of Washington 108: 467-473.

GUÉRIN F.E. 1833. Notice sur les métamorphoses des Cératopogons et description de deux espèces nouvelles, découvertes aux environs de Paris. Annales de la Société Entomologique de France 2: 161-167.

GwizdALSKA-KentZer M. 2011. Biting midges (Diptera: Ceratopogonidae) of the Łęg nad Swelinia reserve. Dipteron 27: 32-39. [in Polish, English abstract].

HACKMAN W. 1980. A check list of Finnish Diptera I. Nematocera and Brachycera (s. str.). Notulae Entomologicae 60: 17-48.

Hagan D.V., Hassold E., Kynde B., Szadziewski R., Thunes K. H., Skartveit J., Grogan W. L. JR. 2000. Biting midges (Diptera: Ceratopogonidae) from forest habitats in Norway. Polish Journal of Entomology 69: 465-476.

HavelKa P. 1976a. Ceratopogoniden-Emergenz am Breitenbach und am Rohrwiesenbach (19711972). Archiv für Hydrobiologie, Suppl. 50: 54-95.

HavelKa P. 1976b. Limnologische und systematische Studien an Ceratopogoniden (Diptera: Nematocera). Beiträge zur Entomologie 26: 211-305. 
HavelKa P. 1978. Blütenbesuchende Ceratopogoniden (Diptera) aus der Umgebung von Tübingen. Beiträge zur Naturkundlichen Forschung in Südwestdeutschland 37: 175-179.

Havelka P. 1979. Situation der Ceratopoginidenforschung auf der Iberischen Halbinsel (Dipt. Ceratopogoniade). Eos 53: 55-74.

Havelka P., Aguilar M. 1999. Ceratopogonidae. [In:] H. Schumann, R. Bährmann, A. Stark (eds). Entomofauna Germanica 2. Checkliste der Dipteren Deutschlands. Studia Dipterologica, supl. 2: 33-38.

HRIBAR J.L., DENSON D.R. 2008. Observations on the co-occurrence of larval Dasyhelea midges (Diptera: Ceratopogonidae) and mosquitoes (Diptera: Culicidae) in container habitats, with a new Florida County record for Dasyhelea pseudoincisurata WAUGH \& WIRTH (Diptera: Ceratopogonidae). Biological Sciences 71: 169-171.

Hribar L.J., Grogan W.L. JR. 2005. New records of biting midges (Diptera: Ceratopogonidae) and frog-biting midges (Diptera: Corethrellidae) from the Florida Keys. Florida Scientist 68: 227-235.

JOHANNSEN O.A. 1931. Ceratopogoninae from the Malayan subregion of the Dutch East Indies. Archiv für Hydrobiologie, Suppl. 9: 403-448.

KaCZOrowska E. 1996. Dasyhelea (s. str.) versicolor (WinNerTZ, 1852) from Poland (Diptera: Ceratopogonidae). Annals of the Upper Silesian Museum, Entomology 6-7: 49-58.

KARL O. 1940. Beiträge zur Kenntnis der Mückenfauna Pommerns. Diptera Nematocera. III. Dohrniana 19: 29-36.

KEILIN D. 1921. On the life history of Dasyhelea obscura WinNERTZ (Diptera, Nematocera, Ceratopogonidae), with some remarks on the parasites and hereditary bacterian symbiont of this midge. Annals and Magazine of Natural History 8: 576-590, pls. 19-20.

KEILIN D. 1927. Fauna of a horse chestnut tree (Aesculus hippocastanum), dipterous larvae and their parasites. Parasitology 19: 368-374.

KIEFFER J.J. 1911. Nouvelles descriptions de chironomides obtenus d'éclosion. Bulletin de la Société d'Histoire Naturelle de Moselle (Metz) 27: 1-60.

KIEFFER J.J. 1913a. Nouvelle étude sur les chironomides de l'Indian Museum de Calcutta. Records of the Indian Museum 9: 119-197, pls. 11-12.

KiefFer J.J. 1913b. Chironomidae et Cecidomyidae. [In:] C. A. Alluaud, R. JEAnNel (eds). Voyage de Ch. Alluaud et R. Jeannel en Afrique orientale (1911-1912). Résultats Scientifiques. Diptera 5: 1-43.

KIEFFER J.J. 1913c. Nouvelle contribution à la connaissance des tendipédides d'Allemagne. Bulletin de la Société d'Histoire Naturelle de Moselle (Metz) 28: 37-44.

KiefFER J.J. 1915. Neue Chironomiden aus Mitteleuropa. Brotéria, Serie Zoologica 13: 65-87.

KIEFFER J.J. 1918. Beschreibung neuer, auf Lazarettschiffen des östlichen Kriegsschauplatzes und bei Ignalino in Litauen von Dr. Horn gesammelter Chironomiden, mit Übersichtstabellen einiger Gruppen von paläarktischen Arten (Dipt.). Entomologische Mitteilungen 7: 35-188.

KiefFER J.J. 1919. Chironomides d'Europe conservés au Musée National Hongrois de Budapest. Annales Historico-Naturales Musei Nationalis Hungarici 17: 1-160.

KIEFFER J.J. 1923. Ceratopogonines recueillis au Sahara Constantinois. Archives de l'Institut Pasteur Algérie 1: 654-683.

KIEFFER J.J. 1924. Chironomides nouveaux ou rares de l'Europe centrale. Bulletin de la Société d'Histoire Naturelle de Moselle (Metz) 30: 11-110.

KIEFFER J.J. 1925a. Chironomiden der Hochmoore Nordeuropas und des östlichen Mitteleuropas. Beiträge zur Kunde Estlands 10: 145-163.

KiEfFER J.J. 1925b. Nouveaux genres et nouvelles espèces de Chironomides piqueurs. Archives de l'Institut Pasteur Algérie 3: 405-430.

KIEFFER J.J. 1925c. Diptères (Nématocères piqueurs): Chironomidae Ceratopogoninae. Fauna de France 11, Paris, pp.: 1-139. 
KIEFFER J.J. 1929. Zur Kenntnis der Chironomiden (Zuckmücken) des Zehlaubruches. Schriften der Physikalisch-Ökonomischen Gesellschaft zu Königsberg 66: 287-312.

KNOZ J. 1996. Ceratopogonidae (Ceratopogonidae). [In:] J.W.A. van ZuiJlen, T.M.J. PeETERS, P.S. VAN WIELINK, A.P.W. VAN ECK, E.H.M. Bouvy (eds). Brand-stof. Een inventarisatie van de entomofauna van het natuurreservaat 'De Brand' in 1990. Tilburg: Insektenwerkgroep KNNVafdeling Tilburg, pp.: 87-88.

KNOZ J. 1997. Ceratopogonidae (Diptera, Nematocera) recorded firstly in the Czech and Slovak Republics. [In:] J. VAŇHARA, R. ROZKOŠNÝ (eds). Dipterologica Bohemoslovaca 8. Folia Facultatis Scientiarum Naturalium Universitatis Masarykianae Brunensis, Biologia 95: 77-87.

KNOZ J. 1998. Ceratopogonidae. [In:] J. VAŇHARA, R. RozKoŠnÝ (eds). Diptera of the Pálava Biosphere Reserve of UNESCO 1. Folia Facultatis Scientiarum Naturalium Universitatis Masarykianae Brunensis, Biologia 99: 113-121.

KnOZ J., BeuK P.L.T. 2002. Ceratopogonidae. [In:] P.L.T. BeuK (ed.). Checklist of the Diptera of the Netherlands. Natuurhistorische bibliotek nr 71, KNNV Uitgeverin, Utrecht, pp.: 103-108.

KnOZ J., OlejNíČEK J., GelBič I. 2004. Biting midges (Diptera, Ceratopogonidae) in the late domestic dump Švábův Hrádek near České Budějovice. Acta Musei Meridionali in České Budějovice 44: 83-86. [in Czech, English abstract].

KONDRACKI J. 2000. Geografia regionalna Polski. Wydawnictwo Naukowe PWN, Warszawa, 441 pp.

KRIVOSHEINA N.P. 1957. A contribution to the knowledge of the heleid fauna (Diptera, Heleidae) of the Oka flood-lands. Entomologičeskoe Obozrenie 36: 418-435. [in Russian, English summary].

Laboulbène A. 1869. Histoire des métamorphoses du Ceratopogon dufouri. Annales de la Société Entomologique de France 9: 157-166.

LeE K.M., Chan K.L. 1985. The biology of Dasyhelea ampullariae in monkey cups at Kent Ridge (Diptera: Ceratopogonidae). Journal of the Singapore National Academy of Science 14: 6-14.

LEE K.M., WIRTH W.W., CHAN K.L. 1989. A new species of Dasyhelea midge reared from drains in Singapore (Diptera: Ceratopogonidae). Proceedings of the Entomological Society of Washington 91: 452-457.

LuKASHEVA N.B. 1987. Ksilofil'nye dvukrylye Severo-Zapadnogo Kavkaza. Akademiâ Nauk SSSR, Zoologičeskij Institut, Leningrad, pp.: 144.

MACFIE J.W.S. 1943. Ceratopogonidae (Diptera) from Egypt. Proceedings of the Royal Entomological Society of London (B) 12: 145-159.

MALlOCH J.R. 1915. The Chironomidae, or midges, of Illinois, with particular reference to the species occurring in the Illinois river. Bulletin of the Illinois State Laboratory of Natural History 10: $275-543$.

Martínez M., Canneva B., Ronderos M.M. 2010. Diptera, Ceratopogonidae, Dasyhelea necrophila Spinelli and Rodriguez, 1999: Detection of eggs in ovitraps, in Uruguay. Check List 6: 239-241.

MAYER K. 1934a. Die Metamorphose der Ceratopogonidae (Dipt.). Ein Beitrag zur Morphologie, Systematik, Ökologie und Biologie der Jugendstadien dieser Dipterenfamilie. Archiv für Naturgeschichte 3: 205-288.

MAYER K. 1934b. Ceratopogoniden aus der Neumark. (Dipt.). Stettiner Entomologische Zeitung 95: 290-294.

MAYER K. 1934c. XCIII. Ceratopogoniden - Metamorphosen (C. Intermediae und C. Vermiformes) der Deutschen Limnologischen Sunda-Expediton, pp.: 166-202.

MAYER K. 1937. Beitrag zur Kenntnis der deutschen Ceratopogoniden (Dipt.) IX. Stettiner Entomologische Zeitung 98: 301-304.

MAYER K. 1959. Zwei Dasyhelea-Arten aus spanischen Salzgärten (Dipt. Heleidae). Deutsche Entomologische Zeitschrift 6: 96-99. 
MCLACHLAN A.J., LAdLE R. 2001. Life in the puddle: behavioural and life-cycle adaptations in the Diptera of tropical rain pools. Biological Reviews 76: 377-388.

MeIGEN J.W. 1804. Klassifikazion und Beschreibung der europäischen zweiflügeligen Insekten. (Diptera LinN.). Erster Band. Abtheilung I. Braunschweig, pp.: 1-152.

MEIGEN J.W. 1830. Systematische Beschreibung der bekannten europäischen zweiflügeligen Insekten. Sechster Theil, Hamm, 404 pp.

Mik J. 1888. Zur Biologie von Ceratopogon MeIG., nebst Beschreibung einer neuen Art dieser Gattung. Wiener Entomologische Zeitung 7: 183-192.

MÜNCHBERG P. 1955. Zur Resistenz der Nematocerenlarven (Diptera) in den Phytotelmen von Dipsacus silvester Houdson. Zeitschrift für Morphologie und Ökologie der Tiere 43: 387-396.

MulLEN G.R., HRIBAR L.J. 1988. Biology and feeding behavior of ceratopogonid larvae (Diptera: Ceratopogonidae) in North America. Bulletin of the Society for Vector Ecology 13: 60-81.

MunSterhjelm G. 1920. Om Chironomidernas Äggläggning och Äggrupper. Acta Societatis pro Fauna et Flora Fennica 47: 1-174.

NAVAI S. 1994. Biting midges of the genus Dasyhelea from Afghanistan, with descriptions of new species (Diptera, Ceratopogonidae). Deutsche Entomologische Zeitschrift 41: 357-399.

OHM P., ReMMert H. 1955. Etudes sur les rockpools des Pyrénées-Orientales. Via et Milieu 6: 194-209.

PALMER A.R. 1957. Miocene arthropods from the Mojave Desert, California. United States Geological Survey Paper 294-G: 237-280.

Petersen F.T., AChim S. 2001. Ceratopogonidae. [In:] F.T. Petersen, R. Meier (eds). A preliminary list of the Diptera of Denmark. Steenstrupia 26: 136-138.

PIERCE W.D. 1966. Fossil arthropods of California. 29. Silicified Miocene pupae of Ceratopogonid flies. Bulletin of the Southern California Academy of Sciences 65: 81-98.

PRZHiboro A.A. 1999. The quantitative characteristics of Diptera (Insecta) of the shallow littoral zone of small lakes in North Karelia. Proceedings of the Zoological Institute RAS 281: 129-134.

PRZHiBORO A.A. 2004. Dipterans (Insecta: Diptera) inhabiting the shallow water zone of lakes Pionerskoye and Pridorozhnoye and quantitative assessment of their abundance. [In:] A.F. Alimova, M.B. IVANOvoJ (eds). Regularities of hydrobiological regime in water bodies of different types. Scientific World, Moscow, pp.: 102-121. [in Russian, English summary].

Przhiboro A.A. 2005. New data on Ceratopogonidae from lake shore habitats in NW Russia (Diptera). Zoosystematica Rossica 14: 165-167.

Przhiboro A.A., BrodskAya N.K. 2006. Mokrêcy (Diptera, Ceratopogonidae) solênyh ozêr Crimean \& Russian Conference on Bloodsucking Insects, Saint-Petersburg, October 24-27, 2006, pp.: $180-182$.

RAmos RAmis D., SAHUQuillo HerRÁiz C. 1983. Primera description para la ciencia de la hembra de Dasyhelea (D.) bicrenata KIEFFER, 1923 (Diptera, Ceratopogonidae). Revista Iberica de Parasitologia 43: 421-422.

Remm H. 1962. The genus Dasyhelea KIEFFER in Estonia (Diptera, Heleidae). Tartu Riikliku Ülikooli Toimetised 120: 108-133. [in Russian, English summary].

REMm H. 1966. On the Lithuanian biting midges (Diptera, Heleidae). Tartu Riikliku Ülikooli Toimetised 180: 53-71. [in Russian, English summary].

Remm H. 1967. On the fauna of Ceratopogonidae (Diptera) in the Caucasus. Tartu Riikliku Ülikooli Toimetised 194: 3-37. [in Russian, English summary].

REMm H. 1969. Mokrecy. [In:] Opredelitel' nasekomyh Evropejskoj čiasti SSSR. „Nauka”, Leningrad, 5: 201-218.

Remm H. 1971. On the Fauna of Ceratopogonidae of South Primorye (Ussuri Land). [In:] Živaja priroda Dal'nego Vostoka. Akademiia Nauk Estonkoi SSR, pp.: 182-220. [in Russian, English summary]. 
Remm H. 1972. New species of Ceratopogonidae (Diptera) from the South Siberia. Tartu Riikliku Ülikooli Toimetised 293: 62-90. [in Russian, English summary].

REMm H. 1973a. Ceratopogonidae. Ergebnisse der zoologischen Forschungen von Dr. Z. Kaszab in der Mongolei (Diptera). Reichenbachia 14: 171-186.

Remm H. 1973b. Beiträge zur Ceratopogoniden-Fauna Ungarns (Diptera) Folia Entomologica Hungarica (Seria Nova) 26, supl.: 349-357.

REMm H. 1979. A catalogue of the Ceratopogonidae (Diptera) of the Estonian S.S.R. Eesti NSV Teaduste Akadeemia Tartu, pp.: 40-60. [in Estonian, Russian and English summary].

Remm H. 1980. New species of the family Ceratopogonidae (Diptera) from the Middle Asia. Tartu Riikliku Ülikooli Toimetised 516: 85-128. [in Russian, English summary].

REMm H. 1981. New synonyms and new names of the Palaearctic Ceratopogonidae (Diptera). Eesti NSV Teaduste Akadeemia Toimetised 30: 27-32.

Remm H. 1988. Family Ceratopogonidae. [In:] Á. Soos, L. PAPP (eds). Catalogue of Palaearctic Diptera. Vol. 3. Akadémiai Kiadó, Budapest, pp.: 11-110.

Remm H. 1993. New species of Ceratopogonidae (Diptera) from the CIS. Izviestia Akademii Nauk Estonii, Biologia 42: 180-200. [in Russian, Estonian and English summary].

Remm H., NAZARMUKhamedov N.A. 1969. New Heleidae species in lower course of the Amu Darya river. Uzbekskij Biologičeskij Žurnal 4: 54-58. [in Russian, English summary].

REMM H., ZHogolev D.T. 1968. Contributions to the fauna of biting-midges (Diptera, Ceratopogonidae) of the Crimea. Entomologičeskoe Obozrenie 47: 826-842. [in Russian, English summary].

Remmert H. 1953. Dasyhelea tecticola n. sp., eine Ceratopogonidae aus Regenrinnen (Diptera: Ceratopogonidae). Beitrag zur Entomologie 3: 333-336.

RiETH J.T. 1915. Die Metamorphose der Culicoidinen (Ceratopogoninen). Archiv für Hydrobiologie, Suppl. 2: 337-442.

Ronderos M.M, Spinelli G.R., DíAz F. 2006. Observations on the immatures of Dasyhelea necrophila SPINELLI \& RoDRíGUEZ in laboratory (Diptera: Ceratopogonidae). Transactions of the American Entomological Society 132: 291-297.

Ronderos M.M, Spinelli G.R., Huerta H., DíAz F. 2003. Immature stages of two Neotropical species of Dasyhelea KIEFFER, 1911 (Diptera: Ceratopogonidae). Transactions of the American Entomological Society 129: 295-308.

SAhuQuillo Herráiz C., Gil Collado J. 1982. Ceratopogonidae (Diptera Nematocera) de Navarra. Revista de Sanidad e Higiene Publica 56: 743-752.

SANNINO L., EsPINOSA B. 2004. The ceratopogonid Dasyhelea tecticola RemmerT (Diptera, Nematocera) new for Italy damages tobacco in seedbed. Informatore Fitopatologico 3: 32-34. [in Italian, English abstract].

SHEPPE W. 1973. Observations on the animal life of some Zambian hot springs. The Ohio Journal of Science 75: 26-29.

SkUSE F.A.A. 1889. Diptera of Australia. Part VI. - The Chironomidae. Proceedings of the Linnean Society of New South Wales 4: 215-311, pls. 11-14.

SpĂTARU P., DAmiAn-GeORgESCU A. 1970. Metamorfoza la Dasyhelea mayeri n. sp. şi Dasyhelea thienemanni n. sp. (Ceratopogonidae, Diptera). Studii şi Cercetari de Biologie, Seria Zoologie 22: 421-431.

STOR̊̊ R. 1937. Fam. Ceratopogonidae. [In:] R. FrEY (ed.). Die Dipterenfauna der Kanarischen Inseln und ihre Probleme. Societas Scientiarum Fennica, Commentationes Biologicae 6: 31-38.

STORÅ R. 1939. Mitteilungen über die Nematoceren Finnlands II. Notulae Entomologicae 19: 16-30.

StrenZKe K. 1950. XI. Anhang. Dasyhelea lithotelmatica n. sp. [In:] A. ThiENEMAnN (ed.). Lunzer Chironomiden. Ergebnisse von Untersuchungen der stehenden Gewässer des Lunzer Seengebietes (Niederösterreich). Archiv für Hydrobiologie, Suppl. 18: 178-187. 
Strobl B.G. 1910. Die Dipteren von Steiermark. II Nachtrag. Mitteilungen des Naturwissenschaftlichen Vereines für Steiermark, (1909) 46: 45-293.

SzADZIEWSKI R. 1983. Flies (Diptera) of the saline habitats of Poland. Polish Journal of Entomology 53: 31-76.

SZADZIEwSKI R. 1985a. A review of the Palaearctic Dasyhelea (Pseudoculicoides) species of the johannseni group, with a description of two new species (Diptera, Ceratopogonidae). Polish Journal of Entomology 55: 79-98.

SZADZIEWSKI R. 1985b. A faunistic review of the Polish biting midges of the genus Culicoides (Diptera, Ceratopogonidae). Polish Journal of Entomology 55: 283-341. [in Polish, English abstract].

SZADZIEWSKI R. 1986. Redescriptions and notes on some Ceratopogonidae (Diptera). Polish Journal of Entomology 56: 3-103.

SzADZIEwSKi R. 1988. Biting midges (Diptera, Ceratopogonidae) from Baltic amber. Polish Journal of Entomology 57: 3-283.

SzADZiewski R. 1991. Ceratopogonidae. [In:] J. RAZOwSKI (ed.). Checklist of Animals of Poland. Vol. II. Wrocław-Warszawa-Kraków, Ossolineum, Polish Academy of Sciences, pp.: 103-109.

SzADZIEwSKi R. 1993. Biting midges (Diptera, Ceratopogonidae) from Miocene Saxonian amber. Acta Zoologica Cracoviensia 35: 603-656.

SzAdZiewski R. 1998. Ceratopogonidae. [In:] B. MerZ, G. BÄChli, J.-P. HAEnNI, Y. GonSETH (eds). Diptera-checklist. Fauna Helvetica 1: 102-104.

SzADZiewski R. 2001. Ceratopogonidae. [In:] M. Gutowski, B. JAROSZEwiCz (eds). Katalog fauny Puszczy Białowieskiej, pp.: 272-273.

Szadziewski R. 2007. Ceratopogonidae. [In:] W. Bogdanowicz, E. Chudzicka, I. Pilipiuk, E. SKIBIŃSKA (eds). Fauna of Poland - characteristic and checklist of species. Vol. II, Muzeum i Instytut Zoologii PAN, pp.: 28-29, 62-65. [in Polish, English summary].

SZADZIEWSKI R., BORKENT A. 2003. New synonyms, combinations and records of biting midges (Diptera: Ceratopogonidae). Polish Journal of Entomology 72: 249-260.

SZADZIEWSKi R., DOMiniaK P. 2006. New synonyms of European Ceratopogonidae (Diptera). Annales Zoologici 56: 139-146.

SZADZIEWSKI R., GIŁKA W., DOMINIAK P. 2007a. A redescription of Forcipomyia squamigera KIEFFER, 1916 in all stages (Diptera: Ceratopogonidae). [In:] T. ANDERSEN (ed.). Contributions to the systematics and ecology of aquatic Diptera - A Tribute to Ole A. Saether. The Caddis Press, pp.: 275-280.

SzADZiEWSKi R., Grogan W.L. JR. 1998. Biting midges from Dominican amber. IV. Species of the tribes Dasyheleini and Forcipomyiini (Diptera: Ceratopogonidae). Polish Journal of Entomology 67: 255-290.

Szadziewski R., Gwizdalska M., DominiaK P. 2004. New records of biting midges in Poland (Diptera: Ceratopogonidae). Entomological News 23: 120.

SzADZIEWSKi R., Gwizdalska-Kentzer M., GiŁKA W. 2011. Order Diptera, family Ceratopogonidae. [In:] H.H. Sheikh Tahnoon Bin Zayed Al Nahyan (patron), A. VAN HARTEN (ed.). Arthropod fauna of the United Arab Emirates. Volume 4. Dar Al Ummah Printing, Publishing, Distribution \& Advertising, Abu Dhabi, UAE, pp.: 636-653.

SZADZIEWSKi R., GWIZDALSKA-KenTZER M., SONTAG E. 2007b. Predatory biting midges of the genus Sphaeromias (Diptera: Ceratopogonidae) in Europe. Polish Journal of Entomology 76: 293-302.

SZAdZiewSki R., KrZYwiŃSKi J., GiŁKA W. 1997. Diptera Ceratopogonidae, Biting Midges. [In:] A.N. NiLSSON (ed.). Aquatic Insects of North Europe - A Taxonomic Handbook. Volume 2. Apollo Books, Kirkeby Sand 19, DK-5771 Stenstrup, Denmark, pp.: 243-263. 
Thienemann A. 1915. Zur Kenntnis der Salzwasser-Chironomiden. Archiv für Hydrobiologie, Suppl. 2: 443-482.

Thienemann A. 1925. Dipteren aus den Salzgewässern von Oldesloe. Mitteilungen der Geographischen Gesellschaft und des Naturhistorischen Museums in Lübeck (2 Ser.) 31: 102-126.

Thienemann A. 1950. Lunzer Chironomiden. Ergebnisse von Untersuchungen der stehenden Gewässer des Lunzer Seengebietes (Niederösterreich). Archiv für Hydrobiologie, Suppl. 18: 1-202.

ThienEmann A. 1954. Chironomus. Leben, Verbreitung und Wirtschaftliche Bedeutung der Chironomiden. Die Binnengewässer 20: 1-834.

Thomsen L.C. 1935. New species of New York State Ceratopogonidae. Journal of the New York Entomological Society 43: 283-296.

TOKUNAGA M. 1940. Biting midges from Japan and neighbouring countries, including Micronesian Islands, Manchuria, North China and Mongolia (Diptera, Ceratopogonidae). Tenthredo 3: 101-165.

TOKUNAGA M. 1963. Some Japanese biting midges breeding in paddy-field water (Diptera, Ceratopogonidae). Scientific Reports of the Kyoto Prefectural University, Agriculture 15: 37-49.

TÓTHOvÁ A., BARTÁK M., KNOZ J. 2004a. Ceratopogonidae of the Bílina and Duchcov environs. [In:] S. Kubík, M. BARTÁK (eds). Dipterologica Bohemoslovaca 11. Folia Facultatis Scientiarum Naturalium Universitatis Masarykianae Brunensis, Biologia 109: 305-313.

Tóthová A., BARTÁK M., KNOZ J. 2004b. Ceratopogonidae of Southern Moravia (NP Podyjí). [In:] P. BITUŠIK (ed.). Dipterologica Bohemoslovaca 12. Acta Facultatis Ecologiae, supl. 1: 143-148.

TóthovÁ A., KnOZ J. 2006. A revised check-list of the biting midges (Diptera, Ceratopogonidae) of the Czech Republic and Slovakia. Studia Dipterologica 13: 103-114.

Tuzet O, ORMiÉRes R. 1964. Stylocystis riouxi n. sp. grégarine parasite de Dasyhelea lithotelmatica STRENZKE 1951 (Diptera Ceratopogonidae). Bulletin de la Société Zoologique de France 89: 163-166.

Vaillant F. 1957. Deux Ceratopogonidae nouveaux de l'Algérie. Bulletin des Travaux Publiés par la Station d'Aquiculture et de Pèche de Castiglione, (N.S.) 1958(9): 263-274.

VALKANOv A. 1941. Über das Auffinden von Anabiotischen Dipteren in Bulgarien. Godišnik na Sofijskija universitet 37: 201-205. [in Bulgarian, German summary].

VAtTiER G. 1964. Dasyhelea adami sp. n. (Diptera, Ceratopogonidae) morphologie et biologie. Bulletin de la Société de Pathologie Exotique 57: 1159-1177.

Waugh W.T., WiRTh W.W. 1976. A revision of the genus Dasyhelea KIEFFER of the Eastern United States North of Florida (Diptera: Ceratopogonidae). Annals of the Entomological Society of America 69: 219-247.

Wilkening A.J., Kline D.L., WiRTh W.W. 1985. An annotated checklist of Ceratopogonidae (Diptera) of Florida with a new synonymy. Florida Entomologist 68: 511-537.

WinNERTZ J. 1852. Beitrag zur Kenntnis der Gattung Ceratopogon MEIGEN. Linnaea Entomologica 6: $1-80$.

WIRTH W.W. 1952. The Heleidae of California. University of California Publications in Entomology 9: $95-266$.

WIRTH W.W. 1976. New names for North American Ceratopogonidae (Diptera). Proceedings of the Entomological Society of Washington 78: 15 .

WIRTH W.W. 1978. New species and records of intertidal biting midges of the genus Dasyhelea KIEFFER from gulf of California (Diptera: Ceratopogonidae). Pacific Insects 18: 191-198.

WIRTH W.W. 1987. A new species of Dasyhelea (Diptera: Ceratopogonidae) from rock pools in the Southwestern United States. Journal of the North American Benthological Society 6: 72-76.

Wirth W.W., BeAVER R.A. 1979. The Dasyhelea biting midges living in pitchers of nepenthes in Southwest Asia (Diptera: Ceratopogonidae). Annales de la Société Entomologique de France (N.S.) 15: 41-52. 
Wirth W.W., MaRston N. 1968. A method for mounting small insects on microscope slides in Canada balsam. Annals of the Entomological Society of America 61: 783-784.

WiRth W.W., Waugh W.T. 1976. Five new Neotropical Dasyhelea midges (Diptera: Ceratopogonidae) associated with culture of cocoa. Studia Entomologica 19: 223-236.

YU Y.-X. 2008. Notes of a new species of Dasyhelea and new record of Stilobezzia (Diptera: Ceratopogonidae). Sichuan Journal of Zoology 27: 165-166. [in Chinese, English abstract].

Yu Y.-X., Liu J.-H., Liu G.-P., Liu Z.-J., Hao B.-S., Yan G., Zhao T.-S. 2006. Ceratopogonidae of China, Insecta, Diptera. Tom 1-2. Military Medical Science Press, Beijing [2005], 1699 pp. [in Chinese, English title].

ZAVŘEL J. 1935. Ze života v nejmenších tůňkách. Přiroda, Brno 28: 1-4.

Zetterstedt J.W. 1850. Diptera Scandinaviae. Disposita et descripta 9, Lundae: 3367-3710.

ZILAHI-SeBESS G. 1931. Anabiotische Dipteren. Archiv für Hydrobiologie 23: 310-329.

ZILAHI-SeBESS G. 1936a. Die Heleiden-Fauna von Szeged und Umgebung. Acta Biologica (Szeged) 4: 35-49.

Zilahi-SeBess G. 1936b. Über Heleiden des Balaton-Gebiets. Arbeiten des Ungarischen Biologischen Forschungs-Institutes, Tihany 8: 196-206. [in Hungarian, German summary].

Zilahi-Sebess G. 1940. Heleiden aus Ungarn. Folia Entomologica Hungarica 5: 10-133. [in Hungarian, German summary].

Received: May 26, 2012

Accepted: August 8, 2012 


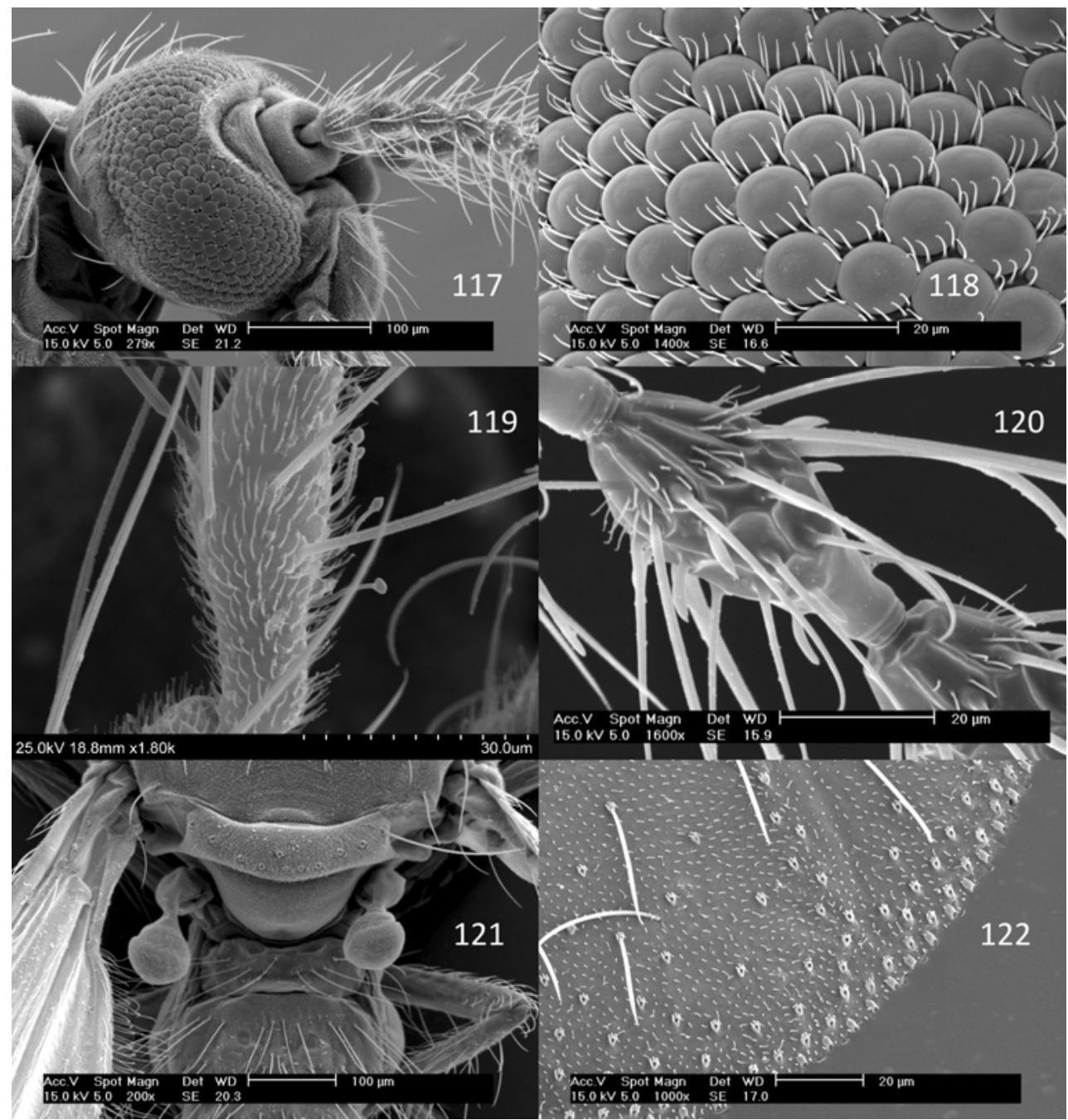

Figs 117-122. SEM photographs, morphology of adult male $(121,122)$ and female $(117,118,119$, 120): 117 - head of Dasyhelea flavifrons (GUÉRIN), 118 - eye of D. flavifrons (GUÉRIN), 119 sensilla capitata on 3rd palpal segment of D. modesta (WINNERTZ), 120 - flagellomeres of D. flavifrons (GUÉRIN), 121 - scutellum, postscutum, halteres and proximal abdominal segments of D. flavifrons (GUÉRIN), 122 - wing membrane of D. flavifrons (GUÉRIN). 


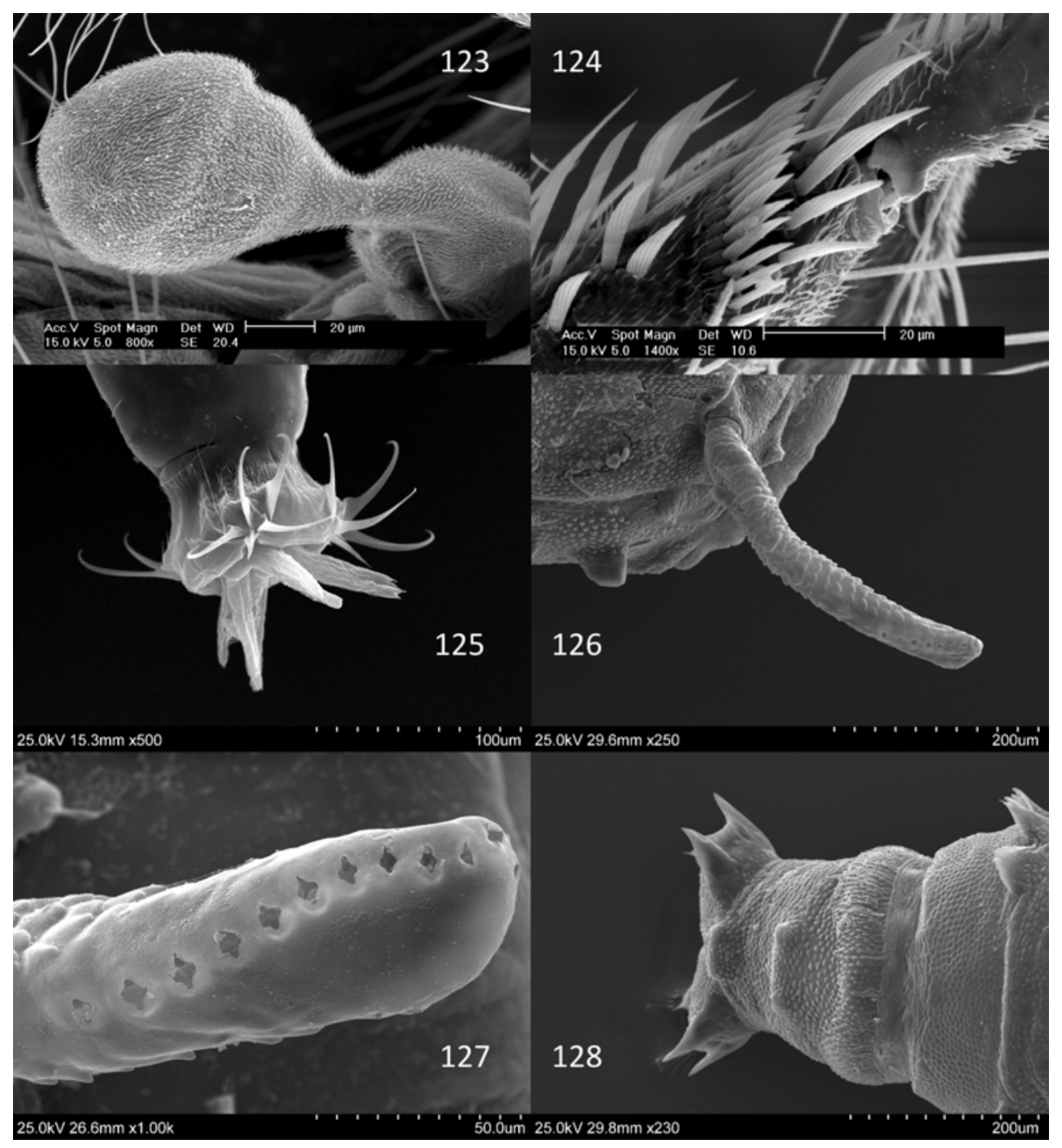

Figs 123-128. SEM photographs, morphology of adult male (123, 124), larva (125) and pupa (126, 127, 128): 123 - haltere with campaniform sensilla of Dasyhelea flavifrons (GUÉRIN), 124 - hind tibial comb of D. calycata REMM, 125 - anal hooks and anal papillae of $D$. flavifrons (GUÉRIN), 126 - respiratoty horn of $D$. bilineata GOETGHEBUER, 127 - spiracles on respiratory horn of D. bilineata Goetghebuer, 128 - posterior processes of anal segment of D. bilineata GOETGHEBUER. 